\title{
Maps of Mineral Deposits Distribution by Provinces in China
}




\section{Miner al legend}

\begin{tabular}{|c|c|c|c|c|c|c|c|c|c|c|c|c|c|c|c|c|c|c|c|c|c|c|c|}
\hline lineral type & \begin{tabular}{|l}
$\begin{array}{l}\text { Super- } \\
\text { large }\end{array}$ \\
\end{tabular} & $\begin{array}{l}\text { Ulura- } \\
\text { large }\end{array}$ & Large & Middie & Small & 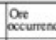 & $\begin{array}{c}\text { Menenli- } \\
\text { and pain }\end{array}$ & Mineral type & $\begin{array}{l}\text { Super- } \\
\text { larget }\end{array}$ & \begin{tabular}{|l|l}
$\begin{array}{l}\text { Ultra- } \\
\text { large }\end{array}$ \\
\end{tabular} & Large & Middle & Small & $\begin{array}{l}\text { Ooe } \\
\text { Oexurus }\end{array}$ & $\mid \begin{array}{c}\text { Minerali- } \\
\text { zed poimt } \\
\mid\end{array}$ & \begin{tabular}{|l|} 
Mineral type \\
\end{tabular} & 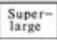 & $\begin{array}{l}\text { Uitura- } \\
\text { large }\end{array}$ & Large & Midale & Small & $\begin{array}{l}\text { Ooe } \\
\text { octure } \\
\text { octe }\end{array}$ & Mancentil \\
\hline Andesite & $\nabla$ & $\nabla$ & $\nabla$ & \$ & $\$$ & 8 & . & Fluerite & III & II & II & 口 & a & a & $\cdot$ & vanadium & $\odot$ & $\odot$ & $\odot$ & $\odot$ & $\odot$ & $\odot$ & $\bullet$ \\
\hline Altapulagix chy & $\nabla$ & $\square$ & $\square$ & $\square$ & $\square$ & 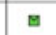 & 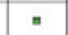 & Jade & 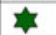 & $\star$ & 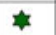 & \# & . & * & - & Caromium & $\odot$ & $\odot$ & $\odot$ & $\odot$ & $\odot$ & - & 0 \\
\hline Cretaceous & 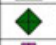 & $\diamond$ & $\nabla$ & 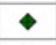 & 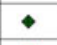 & - & & Mica & $\nabla$ & $\theta$ & & & & - & . & Manganese & P & $\odot$ & $\odot$ & • & $\bullet$ & - & - \\
\hline Dolomite & D & 西 & II & II & 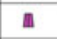 & $a$ & . & Feldspar & $\diamond$ & $\diamond$ & 8 & $\diamond$ & - & - & - & Titanium & C & $\odot$ & $\odot$ & $\bullet$ & $\bullet$ & $\bullet$ & - \\
\hline Slate & 四 & 血 & 血 & III & III & III & $\bar{n}$ & Native solphur & $\triangle$ & $\triangle$ & & $\Delta$ & $\Delta$ & 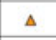 & 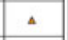 & Iron & $\odot$ & $\odot$ & $\odot$ & P & $\bullet$ & - & - \\
\hline Gem & $\downarrow$ & $\star$ & $\star$ & 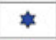 & $*$ & $*$ & - & Topax & 0 & 0 & & & 0 & - & 。 & Tellurium & $\theta$ & $\theta$ & $\theta$ & & $\theta$ & - & - \\
\hline Iceland spar & $\Delta$ & $\Delta$ & $\Delta$ & $\Delta$ & $\Delta$ & $\Delta$ & 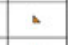 & Diabase & 므 & $\square$ & & & & " & & dmium & $\theta$ & $\theta$ & $\theta$ & $\Theta$ & $\theta$ & $\ominus$ & - \\
\hline Traehyle & 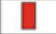 & ] & 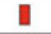 & $=$ & ॥ & " & ' & Volcanic ast & & $\boldsymbol{Z}$ & 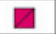 & च & a & a & - & Hafnium & 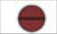 & P & • & • & - & - & - \\
\hline Marble & 曰 & 日 & 日 & 日 & 日 & 是 & в & Ginder & $\nabla$ & $\bar{\Delta}$ & 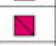 & $\Delta$ & I & 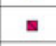 & . & Gallium & $\theta$ & $\theta$ & $\theta$ & $\theta$ & $\theta$ & $\theta$ & - \\
\hline Iodine & $\Phi$ & $\Phi$ & ( & - & w & - & - & Potassium salt & 0 & - & & - & - & 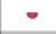 & - & Rhenium & & P & P & & - & - & - \\
\hline Tourmaline & $\nabla$ & 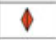 & $\checkmark$ & 1 & 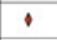 & $\cdot$ & 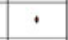 & Amphibolite & $\square$ & I & & E & - & a & . & Thallium & & P & P & & - & $\bullet$ & - \\
\hline Witberite & $\triangle$ & $\triangle$ & $\Delta$ & $\Delta$ & $\Delta$ & $\Delta$ & $\triangle$ & Diamoosd & 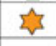 & $\underline{s}$ & 5 & $\Leftrightarrow$ & 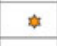 & 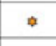 & - & Selenium & $\theta$ & $\theta$ & $\theta$ & & $\theta$ & $\theta$ & $\cdot$ \\
\hline Calcite & & $\square$ & 口 & घ & - & - & 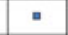 & Mineral salt & $\bullet$ & $\nabla$ & $\nabla$ & $\nabla$ & - & 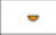 & - & Indium & $\theta$ & $\theta$ & $\theta$ & & $\Theta$ & $\theta$ & • \\
\hline Zeolite & $\Delta$ & $\Delta$ & $\Delta$ & $\Delta$ & $\Delta$ & $\Delta$ & $\triangle$ & Disthene & $\square$ & $\square$ & $\square$ & L & - & - & - & Germanium & $\theta$ & $\theta$ & $\theta$ & & $\theta$ & $\theta$ & - \\
\hline Pink quartz & $\Delta$ & $\Delta$ & $\Delta$ & $\Delta$ & $\Delta$ & A & - & \begin{tabular}{|l|} 
Blue asbestios \\
\end{tabular} & $\downarrow$ & $\downarrow$ & 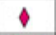 & - & - & . & . & Bismuth & & 0 & - & 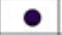 & • & 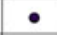 & - \\
\hline Peridotite & $\nabla$ & $\nabla$ & $\nabla$ & $v$ & $v$ & $\mathbf{v}$ & 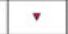 & \begin{tabular}{|l|} 
Rectorite clay \\
\end{tabular} & $\triangle$ & Q & $\omega$ & E & 口 & ㅁ & 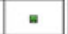 & Mercery & & (. & (๑) & - & • & $\bullet$ & - \\
\hline damantine spar & 0 & 0 & 0 & 0 & 0 & $\circ$ & $\circ$ & Phosphate & $\nabla$ & $\nabla$ & $\nabla$ & $\nabla$ & $\nabla$ & - & $\nabla$ & obalt & & ○ & - & & 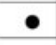 & $\bullet$ & - \\
\hline Kaoline & $\infty$ & 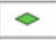 & $\infty$ & 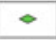 & - & - & $\cdot$ & Magnesite & 日 & 日 & E & $E$ & a & a & - & Alumina & & 0 & 0 & 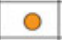 & 0 & 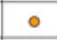 & 。 \\
\hline Wollastonite & $\diamond$ & $\odot$ & $<$ & $\bullet$ & - & $\cdot$ & $\cdot$ & Pyrite & $\triangle$ & $\triangle$ & $\triangle$ & $\Delta$ & $\Delta$ & $\Delta$ & $\Delta$ & Magnesium & & 0 & 0 & & • & - & • \\
\hline Diatomite & 田 & 回 & 㖆 & 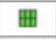 & 曲 & 田 & 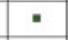 & Agate & $\downarrow$ & 4 & 4 & 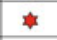 & * & 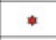 & • & \begin{tabular}{|l|} 
Molybdenum \\
\end{tabular} & 0 & 0 & 0 & & 0 & c & 。 \\
\hline Sepiotite clay & 曲 & 困 & 曹 & 田 & 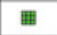 & II & - & Granodiorite & 0 & P & $=$ & 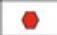 & - & - & - & Nickel & D & 0 & 0 & & 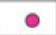 & • & - \\
\hline 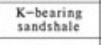 & $\nabla$ & V & V & $\nabla$ & $\nabla$ & $\nabla$ & 2 & Vein quartx & D & 11 & . & & & - & $\cdot$ & ead & P & 0 & 0 & & & & $\circ$ \\
\hline $\mathrm{K}$-bearing rock & $\nabla$ & $\nabla$ & $\nabla$ & $\sqrt{2}$ & $v$ & $\nabla$ & $\checkmark$ & Mirabilite & - & - & 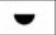 & 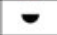 & 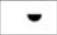 & 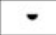 & 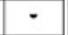 & Stibium & 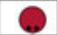 & (1) & $\bullet$ & & 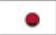 & • & - \\
\hline Obsidian & A & a & 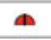 & 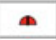 & - & - & 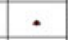 & Muesesium sultane & $\checkmark$ & 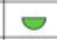 & 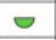 & $\sigma$ & - & 0 & - & copper & 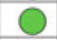 & 0 & 0 & & 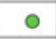 & 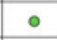 & 。 \\
\hline Andalusite & & D & E & a & 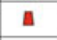 & a & $\cdot$ & Alunite & $\Delta$ & $\Delta$ & 4 & 4 & 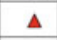 & $\Delta$ & 4 & 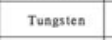 & 0 & 0 & - & & $\bullet$ & - & • \\
\hline Grasite & & D & U & 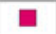 & a & " & 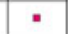 & Nitratine & $\sigma$ & $\nabla$ & 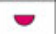 & - & - & - & $\cdot$ & Stannum & 0 & 0 & ? & & 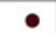 & - & • \\
\hline Talc & $\nabla$ & 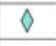 & $\theta$ & -1 & - & $\cdot$ & - & \begin{tabular}{|l|} 
Refractory clay \\
\end{tabular} & 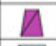 & $\Delta$ & Z & $E$ & E & - & 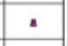 & Zine & 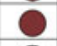 & 0 & - & & 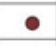 & 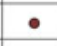 & - \\
\hline Gneiss & $\infty$ & $<$ & $<$ & $\diamond$ & 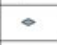 & $\cdot$ & 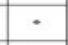 & Mar1 & $\nabla$ & $\nabla$ & $\nabla$ & $\Delta$ & $\Delta$ & $\sigma_{0}$ & 8 & Plastinum & $\theta$ & (1) & $\theta$ & 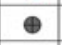 & $\bullet$ & - & - \\
\hline Other clay & & $\square$ & 둘 & $\square$ & ㅁ & - & $=$ & Mudstione & 田 & 田 & 2 & 본 & - & 田 & - & Osmium & $\oplus$ & $\oplus$ & $\oplus$ & & $\oplus$ & $\oplus$ & • \\
\hline Sandstiose & & $\Delta$ & 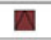 & E & a & . & - & Tuff & $\nabla$ & $\mathbf{\nabla}$ & $\Delta$ & $\Sigma$ & 国 & - & 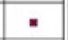 & Goid & $\oplus$ & $\oplus$ & (†) & & $\oplus$ & 4 & - \\
\hline Diorite & & $\square$ & 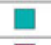 & - & $\square$ & . & 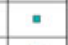 & Boron & - & - & - & 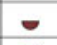 & - & - & - & Ruthenium & (1) & (1) & - & & 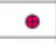 & $\bullet$ & - \\
\hline Serpentinite & $\nabla$ & $\nabla$ & $\nabla$ & $\nabla$ & $\nabla$ & $\checkmark$ & $\checkmark$ & ntonite & $凶$ & 区 & 区 & $\mathbb{\Delta}$ & & $-\pi$ & - & Palladium & $\oplus$ & $\oplus$ & $\oplus$ & & - &. & • \\
\hline Arsenic & $\triangle$ & $\triangle$ & $\triangle$ & $\Delta$ & $\Delta$ & $\Delta$ & $\triangle$ & terite & 目 & 目 & 目 & 国 & 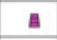 & 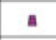 & $\cdot$ & dium & $\theta$ & $\theta$ & $\oplus$ & & $\oplus$ & - & - \\
\hline$G_{\text {ypsum }}$ & $\theta$ & 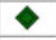 & 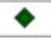 & $\bullet$ & 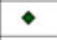 & - & • & ide & $\bullet$ & $<$ & $\bullet$ & - & - & $\cdot$ & $\cdot$ & iver & $\oplus$ & $\oplus$ & $\oplus$ & & $\oplus$ & $\oplus$ & • \\
\hline Limestione & $\square$ & $\square$ & [ & $\square$ & 口 & 口 & 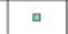 & Tremolite & 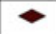 & $\bullet$ & $\bullet$ & 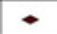 & 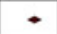 & 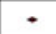 & $\cdot$ & Zirconium & $\theta$ & $\theta$ & $\ominus$ & $\theta$ & $\bullet$ & - & - \\
\hline Garnet & $\downarrow$ & $\star$ & $\star$ & $\star$ & * & * & - & Nepteline syetile & - & - & $\bullet$ & - & $\bullet$ & - & - & Lithium & $\theta$ & P & $\ominus$ & & $\bullet$ & $\bullet$ & - \\
\hline Asbestos & $\vartheta$ & $\hat{4}$ & $\nabla$ & 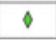 & $\circ$ & $\cdot$ & . & Bromine & $\checkmark$ & $\checkmark$ & $\nabla$ & c & $\sigma$ & 0 & - & Niobium & $\theta$ & $\ominus$ & $\theta$ & & $\ominus$ & $\theta$ & - \\
\hline Grapsite & 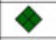 & $\theta$ & $\bullet$ & $\bullet$ & $\bullet$ & $\bullet$ & • & Basalt & 日 & 日 & 日 & 目 & 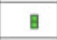 & : & . & Beryllium & $\theta$ & $\theta$ & $\theta$ & & $\ominus$ & - & - \\
\hline Quartrite & M & $\nabla$ & D & 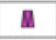 & a & 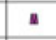 & $\cdot$ & Figment misenal & 目 & 目 & 目 & 目 & E & ㅍ & - & Rubidium & $\theta$ & $\theta$ & $\theta$ & & $\theta$ & $\theta$ & - \\
\hline Quartz crystal & $\Delta$ & $\mathbf{\Delta}$ & $\mathbf{1}$ & 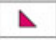 & 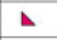 & $\Delta$ & - & Pyrophyllit & $\theta$ & $\theta$ & $\theta$ & & & ${ }^{\circ}$ & 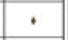 & Cesit & $\theta$ & $\theta$ & 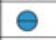 & & $\theta$ & - & $\bullet$ \\
\hline Pitchstione & a & a & 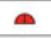 & . & - & - & . & Shale & 日 & 日 & 是 & E & & 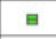 & " & Strontium & 0 & $\theta$ & 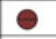 & & $\bullet$ & 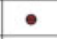 & - \\
\hline Cetramic clay & 曰 & E & 日 & 日 & E & e & - & Illite clay & 0 & 0 & 0 & - & 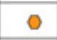 & 0 & - & Tantalum & $\theta$ & $\theta$ & $\theta$ & $\nabla$ & $\theta$ & $\theta$ & - \\
\hline Trons & - & - & - & - & - & - & $\cdot$ & Penlite & - & - & - & ๑ & - & - & . & cosel & = & $=$ & $=$ & - & - & - & $\cdot$ \\
\hline fal quartx & $\diamond$ & 4 & 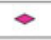 & - & 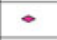 & $\bullet$ & - & Vermiculite & $\Delta$ & $\downarrow$ & $\checkmark$ & & 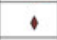 & 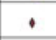 & $\cdot$ & Stone coal & - & $=$ & 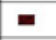 & 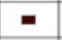 & $=$ & - & $\cdot$ \\
\hline an 10 & 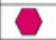 & 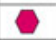 & 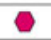 & $\bullet$ & - & $\bullet$ & • & Barite & & & & $\Delta$ & $\Delta$ & $\Delta$ & $\Delta$ & Natural aspasalt & $\downarrow$ & $\checkmark$ & $\checkmark$ & $\checkmark$ & $\bullet$ & $\cdot$ & • \\
\hline
\end{tabular}

\section{Geographical legend}

$\star$ Beijing Capital

- Guangzhou $\begin{gathered}\text { Provincial administrative } \\ \text { center }\end{gathered}$

- Foshan $\begin{aligned} & \text { Administrative center of } \\ & \text { a prefecture-level city }\end{aligned}$

Administrative center of

a autonomous prefecture
or location of a regionor league-level administra-

$\circ$ Yanji
National boundary

Boundary of a province, municipality

Boundary of a special
administrative region

Boundary of a prefecture-level
River

Lake, reservoir

Canal 
Deposit distribution map of Beijing Shi, China

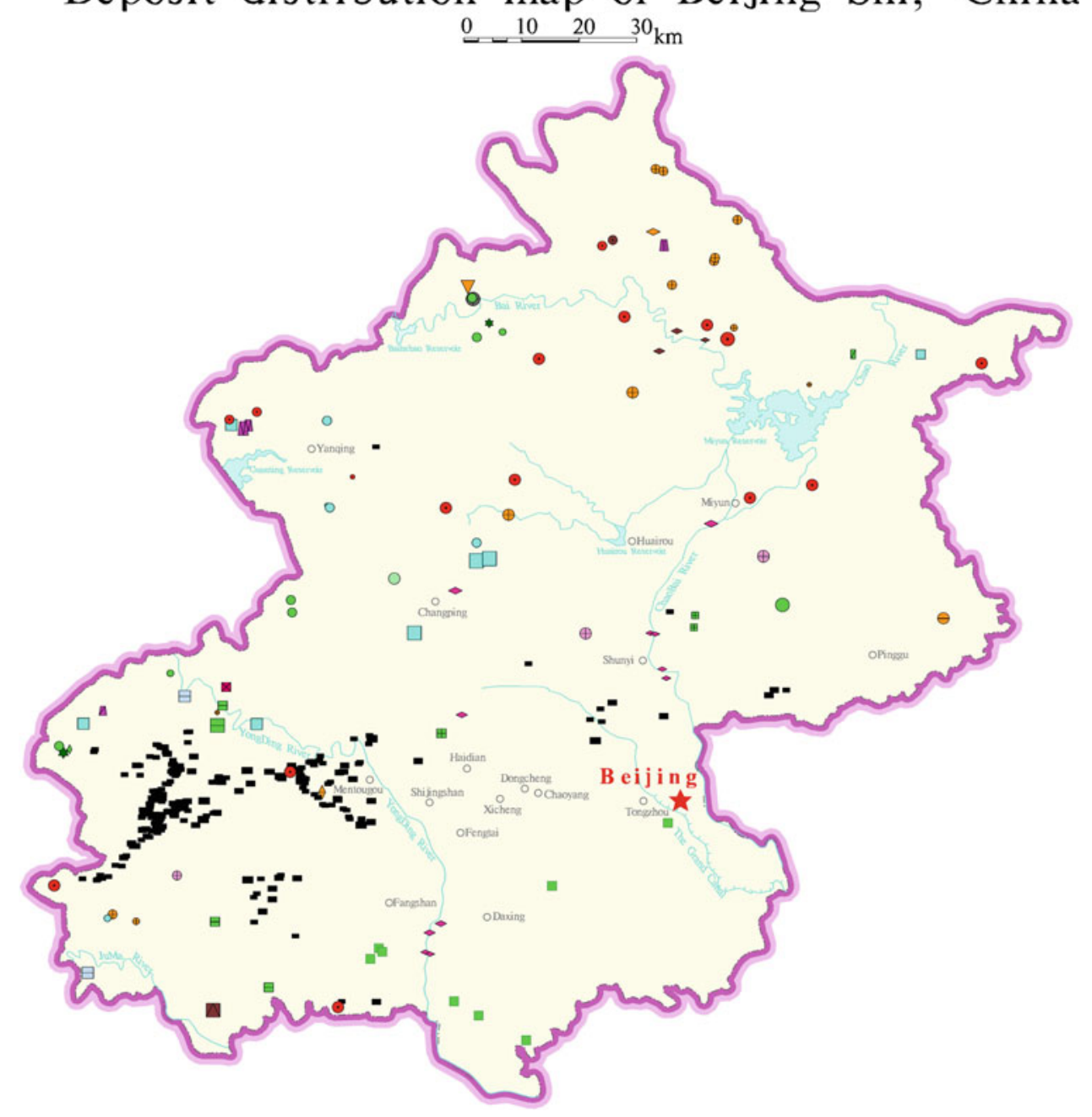


12

4 Maps of Mineral Deposits Distribution by Provinces in China

Deposit distribution map of Tianjin She, China

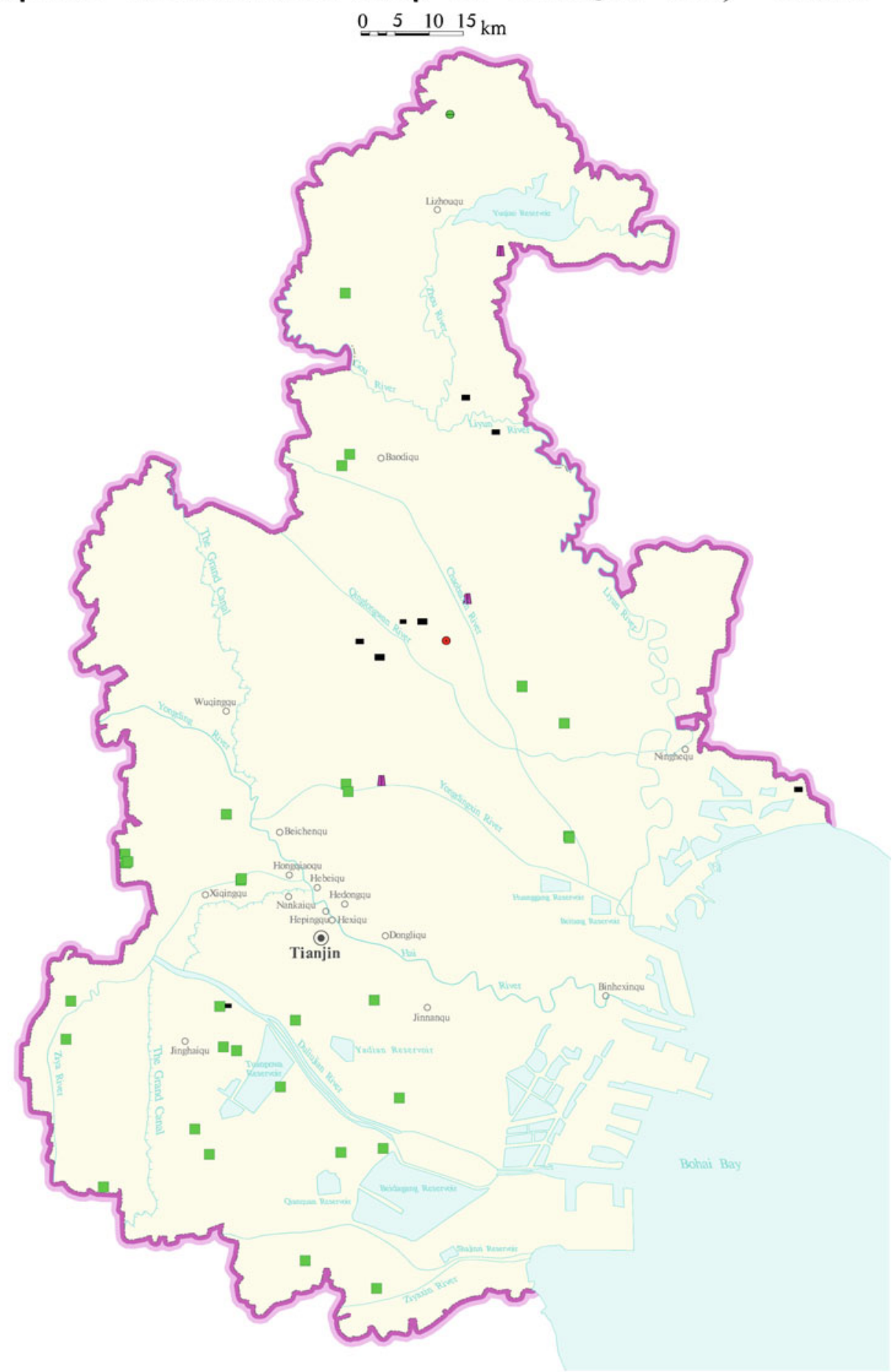


Deposit distribution map of Hebei Province

(metallic minerals)

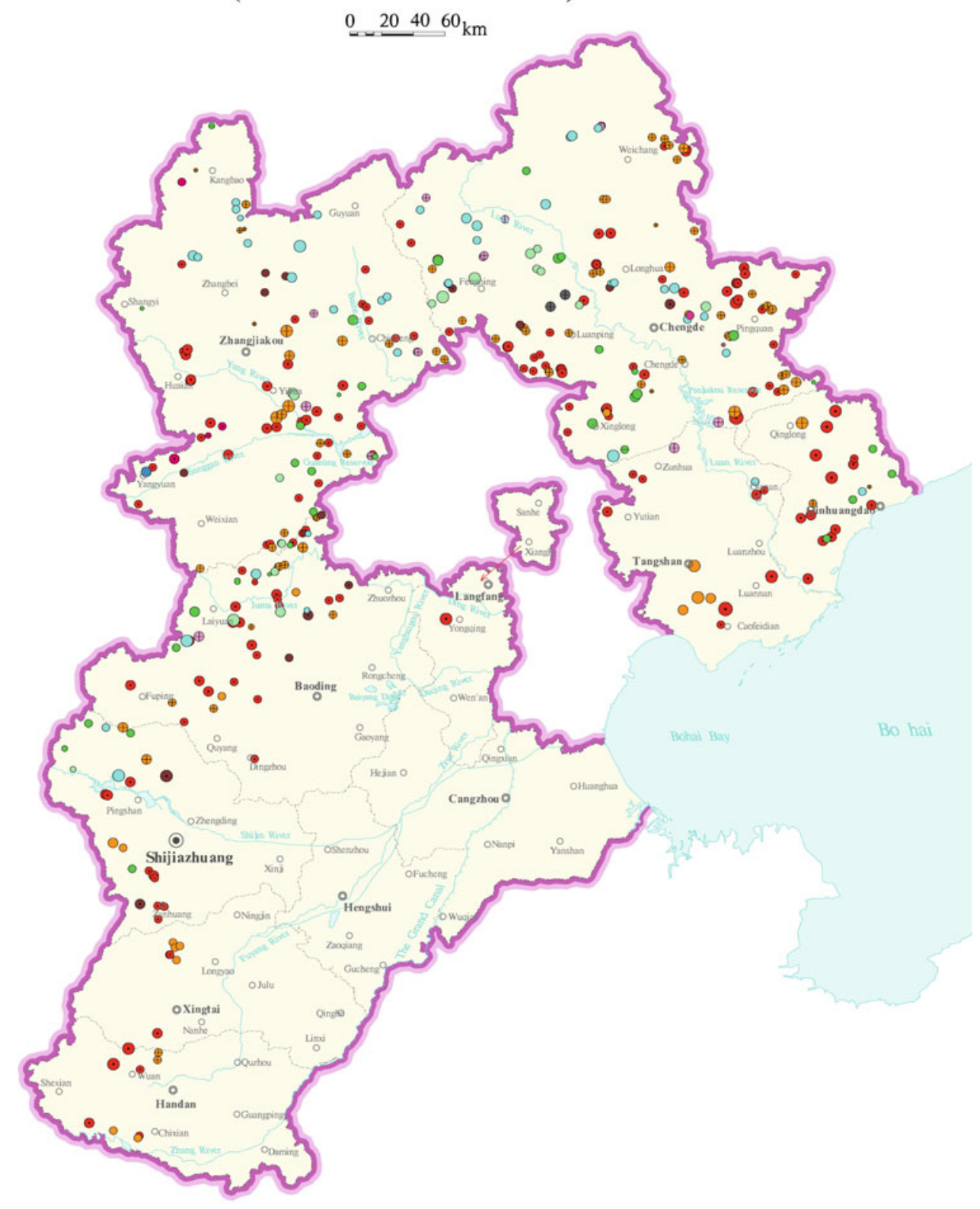


Deposit distribution map of Hebei Province

( energy minerals)

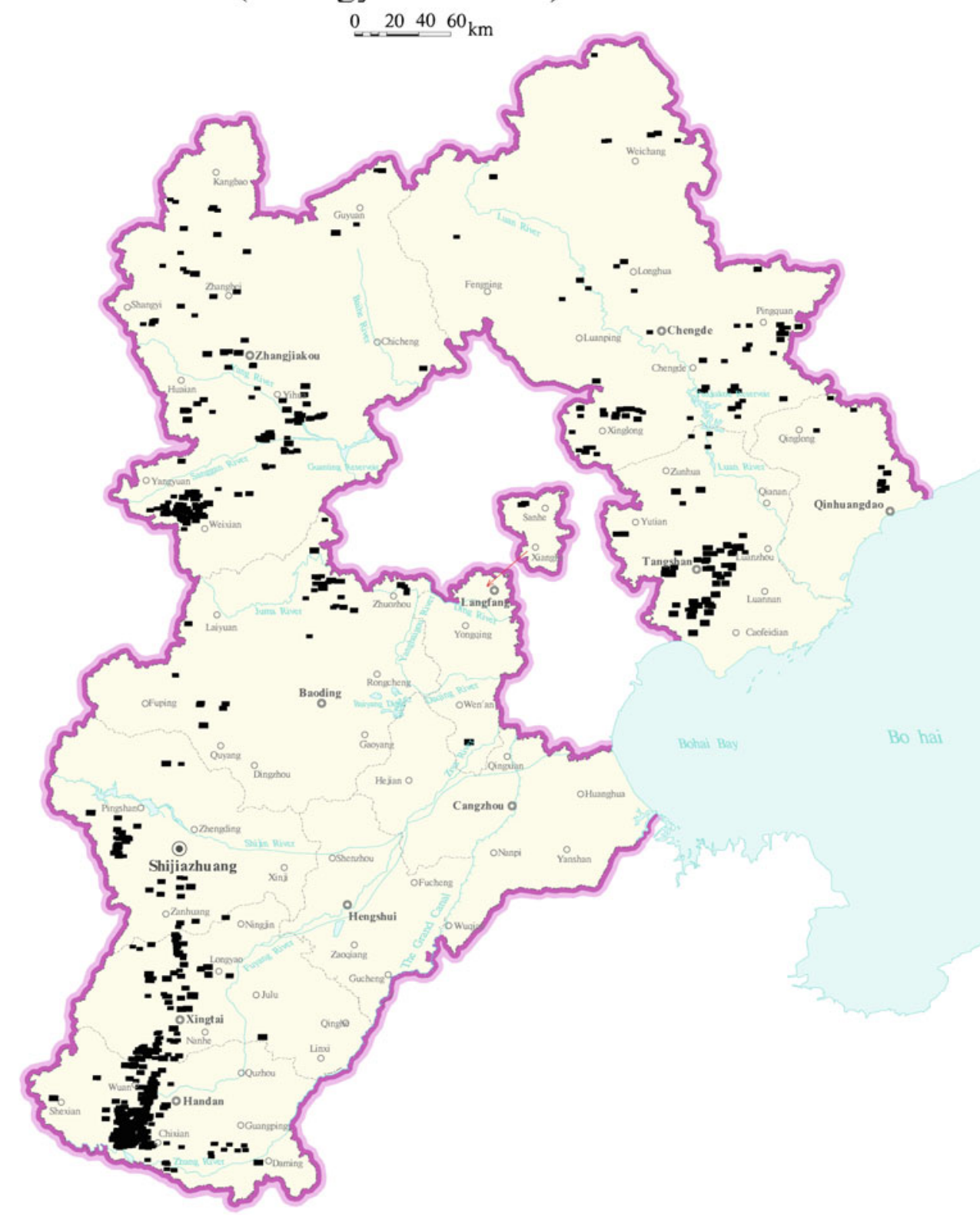


4 Maps of Mineral Deposits Distribution by Provinces in China

15

Deposit distribution map of Hebei Province (non-metallic minerals)

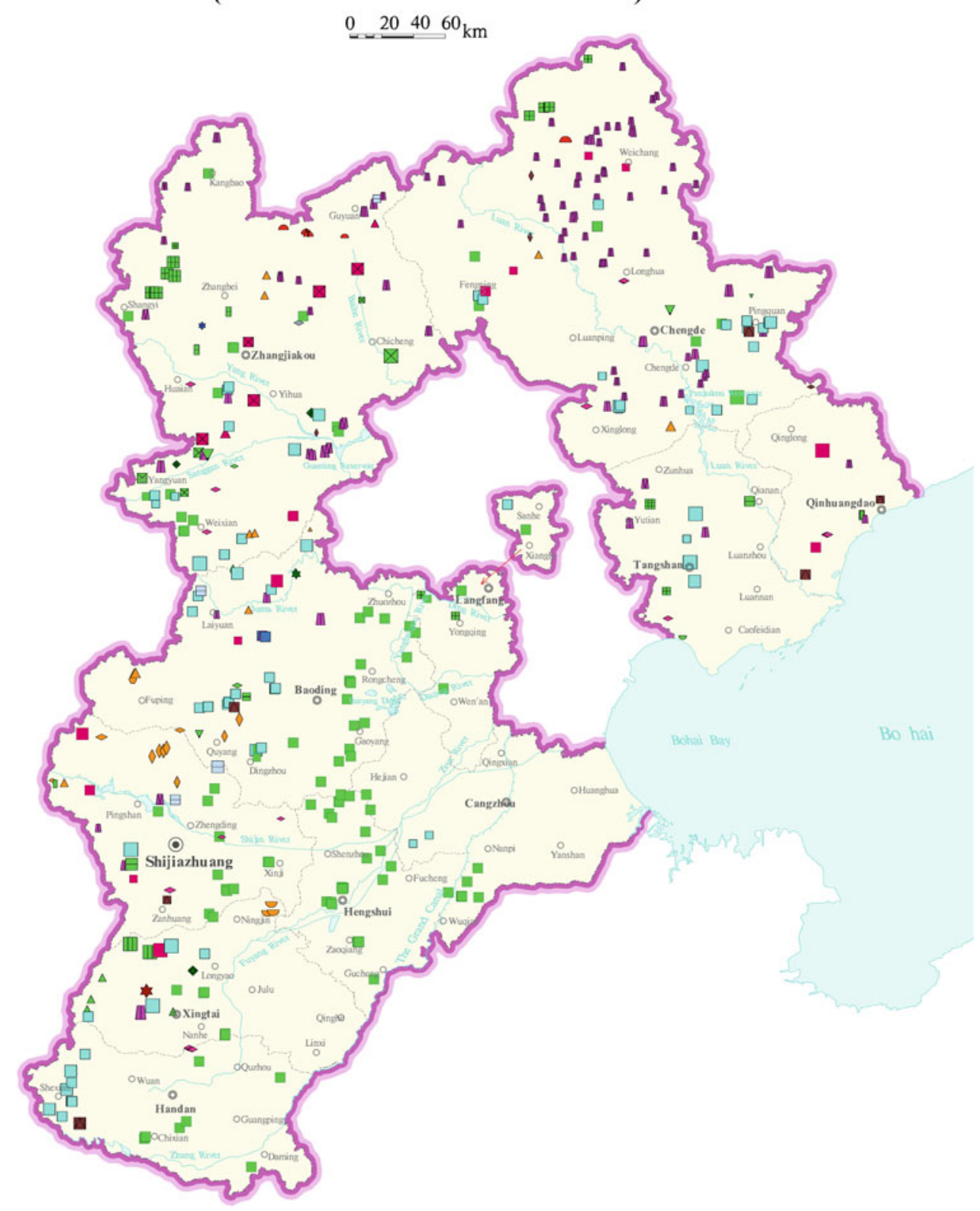




\section{Deposit distribution map of Shanxi Province}

(metallic minerals)

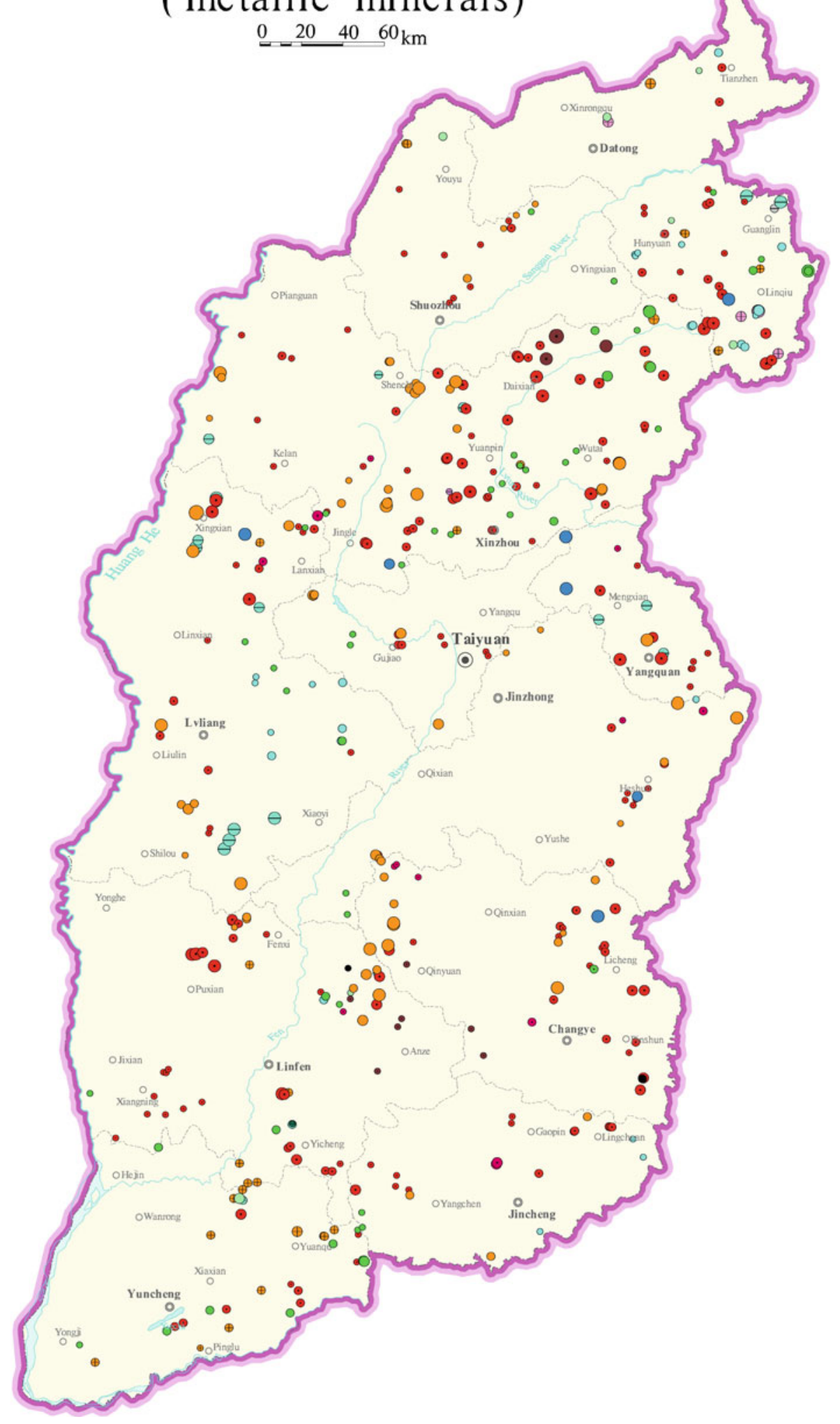


Deposit distribution map of Shanxi Province

(energy minerals)

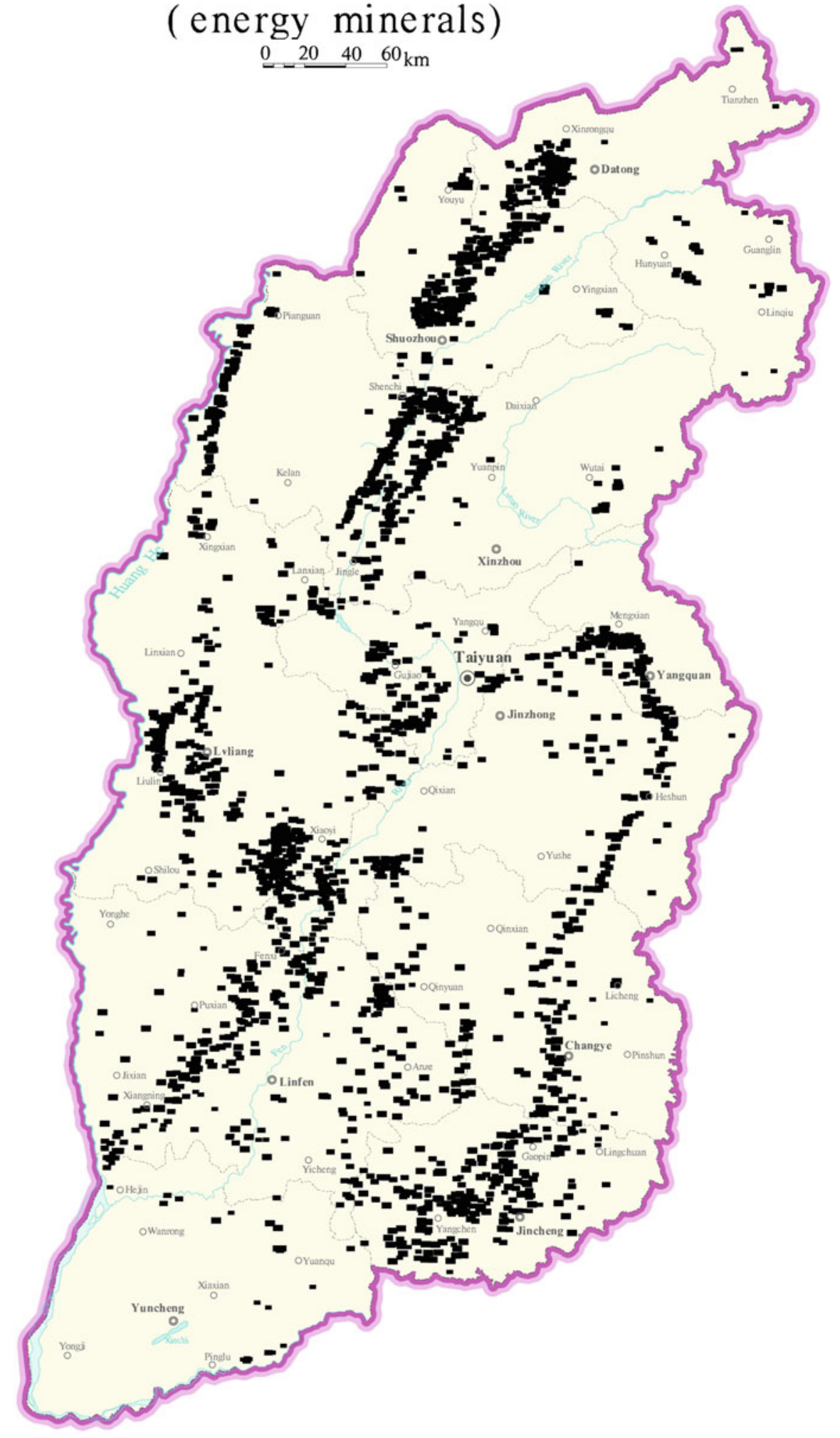




\section{Deposit distribution map of Shanxi Province}

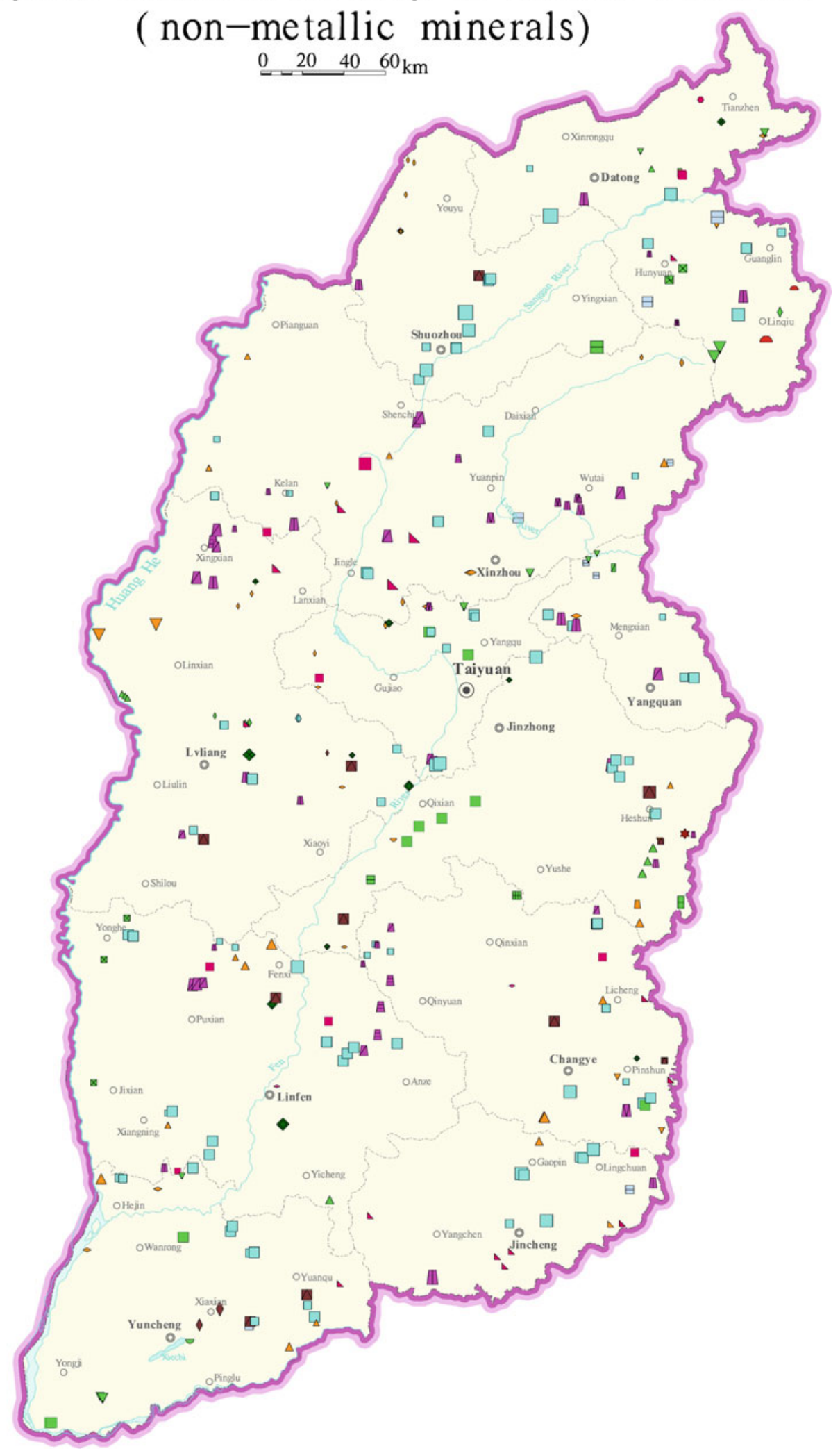


Deposit distribution map of Neimongolzizhiqu Region ( metallic minerals)

$\underline{0} \quad \begin{array}{lll}100 \quad 200 \quad 300 & & \end{array}$
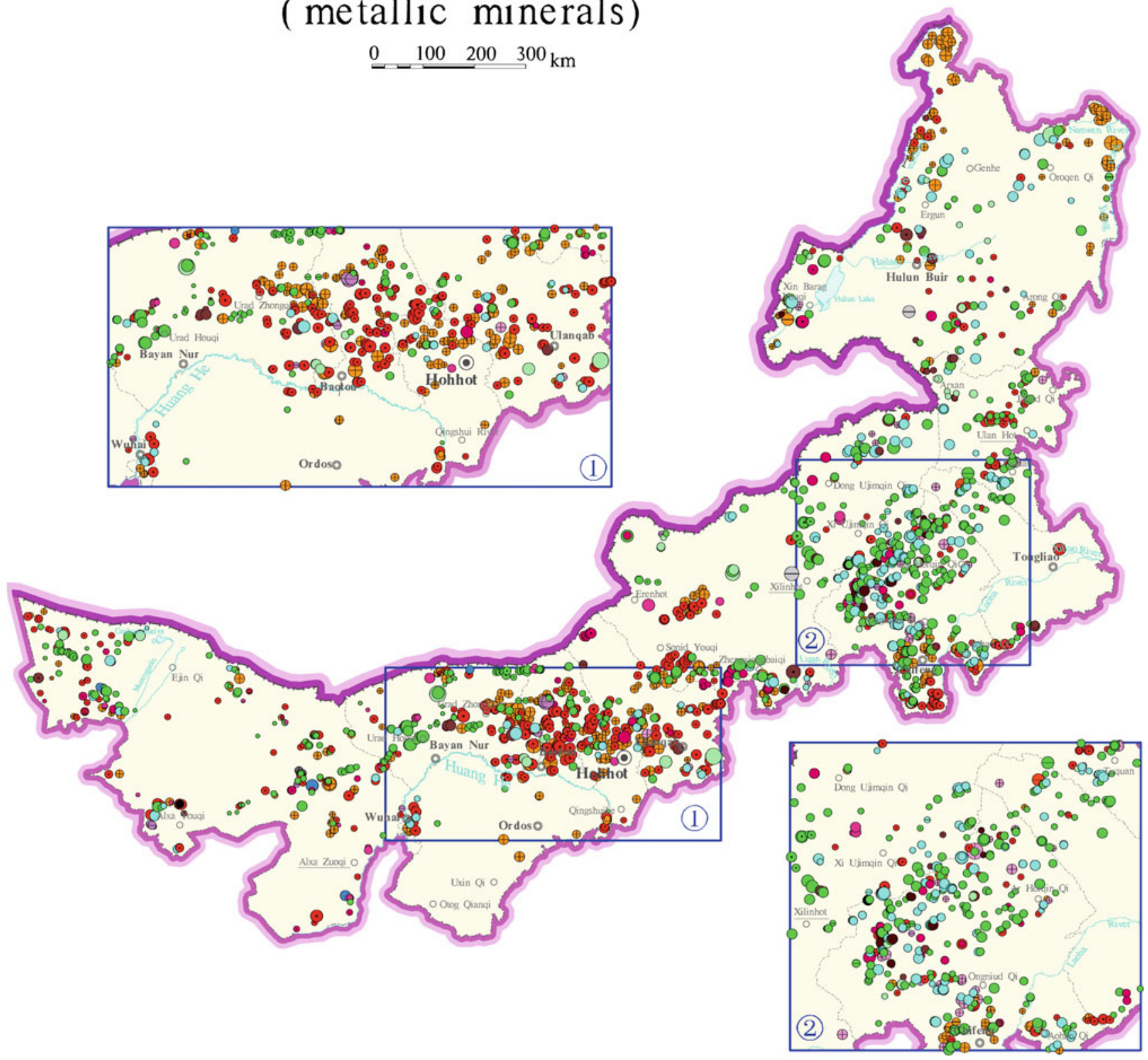
Deposit distribution map of Neimongolzizhiqu Region ( energy minerals)

$$
\underline{0}-\underline{100 \quad 200 \quad 300} \mathrm{~km}
$$

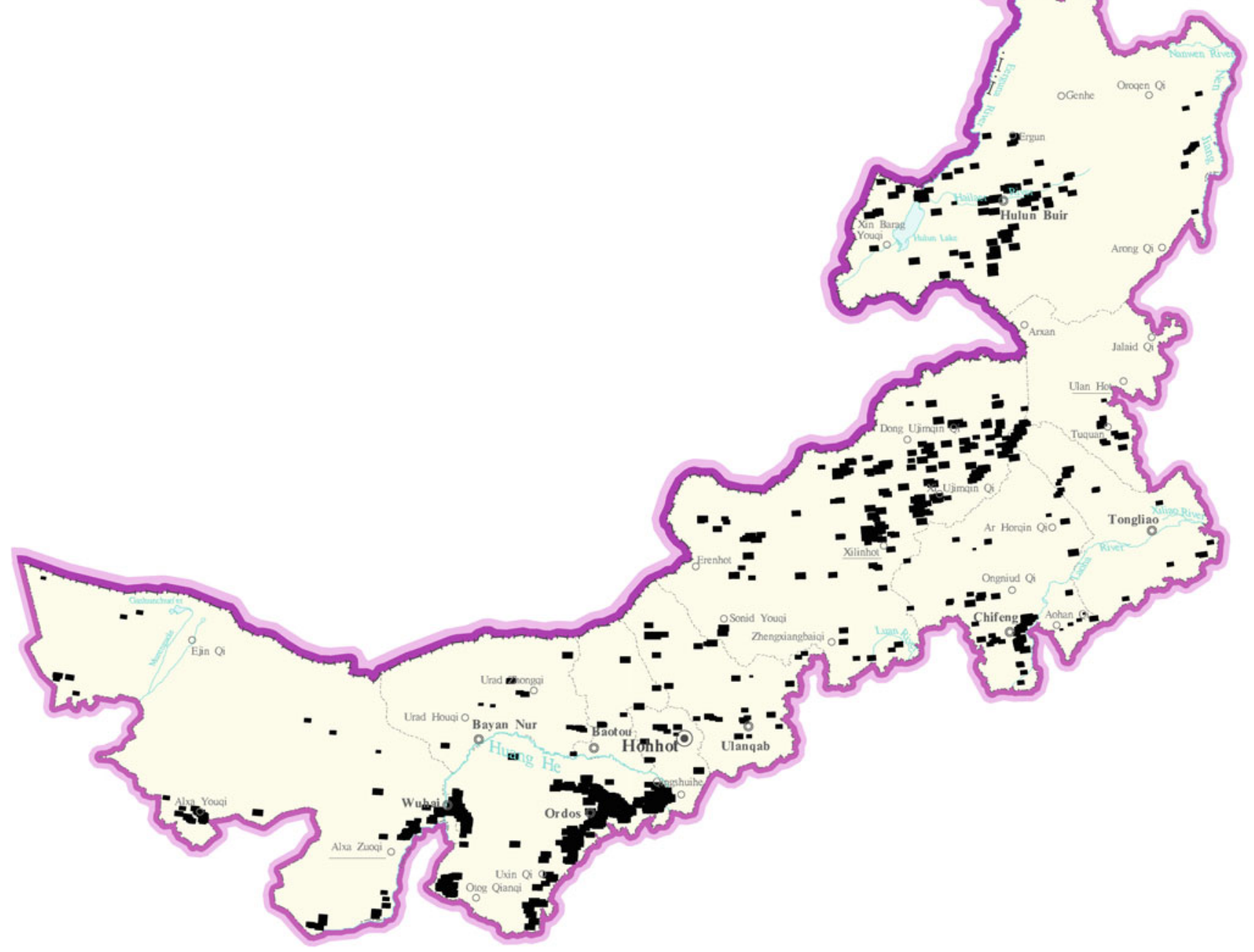


Deposit distribution map of Neimongolzizhiqu Region (other non-metallic minerals)

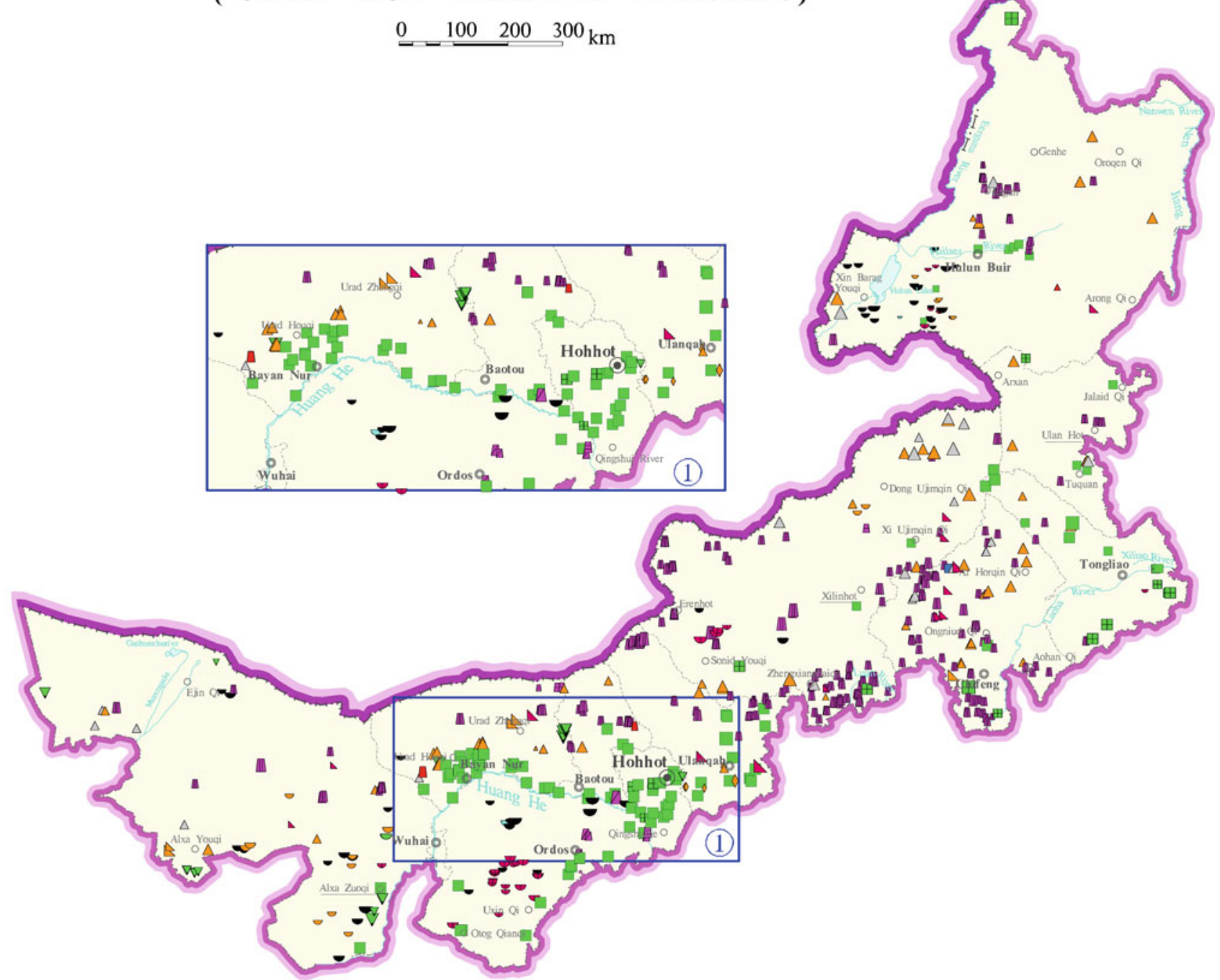


Deposit distribution map of Neimongolzizhiqu Region (building materials)
$0 \quad 100200300 \mathrm{~km}$

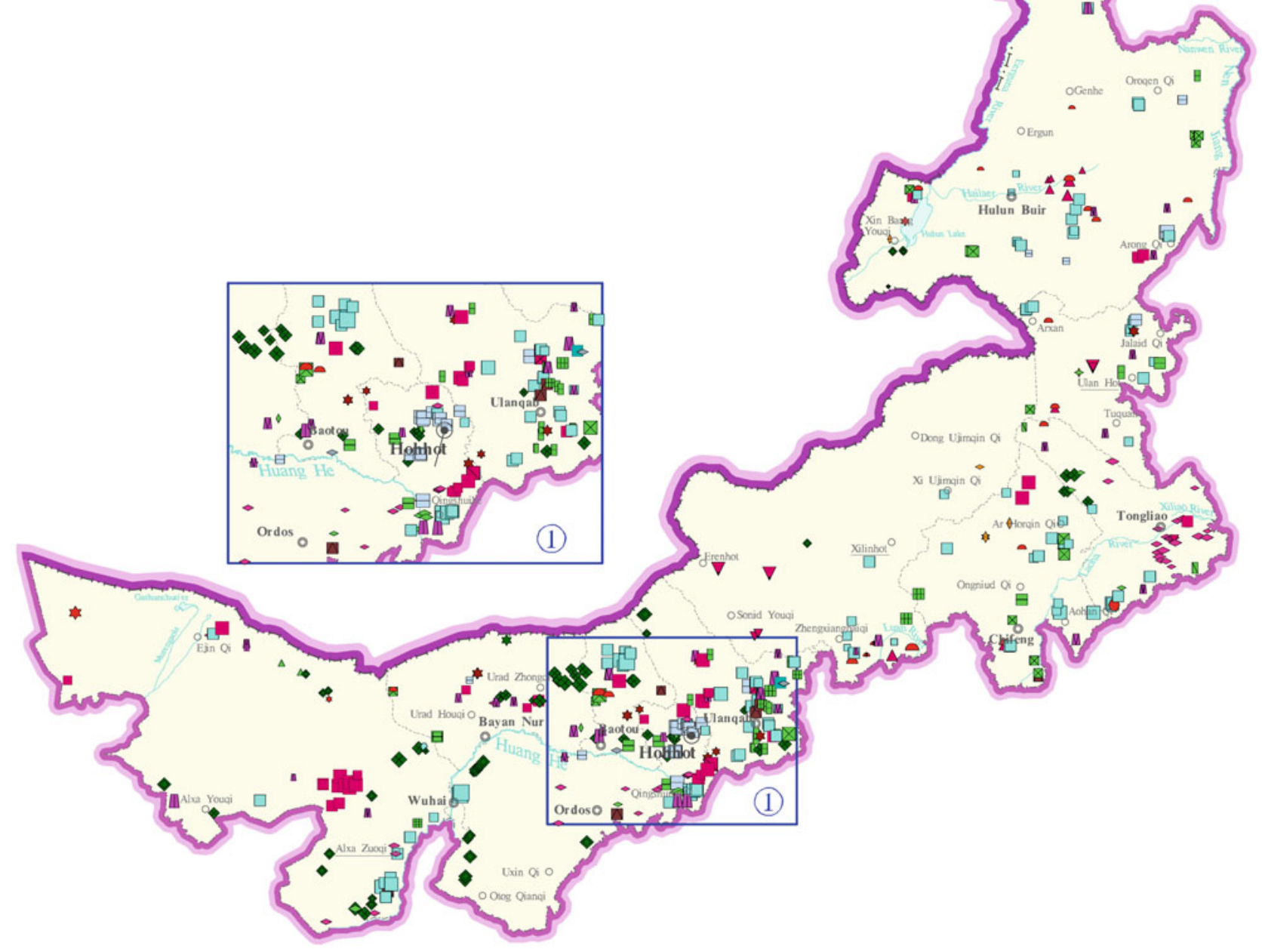




\section{Deposit distribution map of Liaoning Province} (metallic minerals)

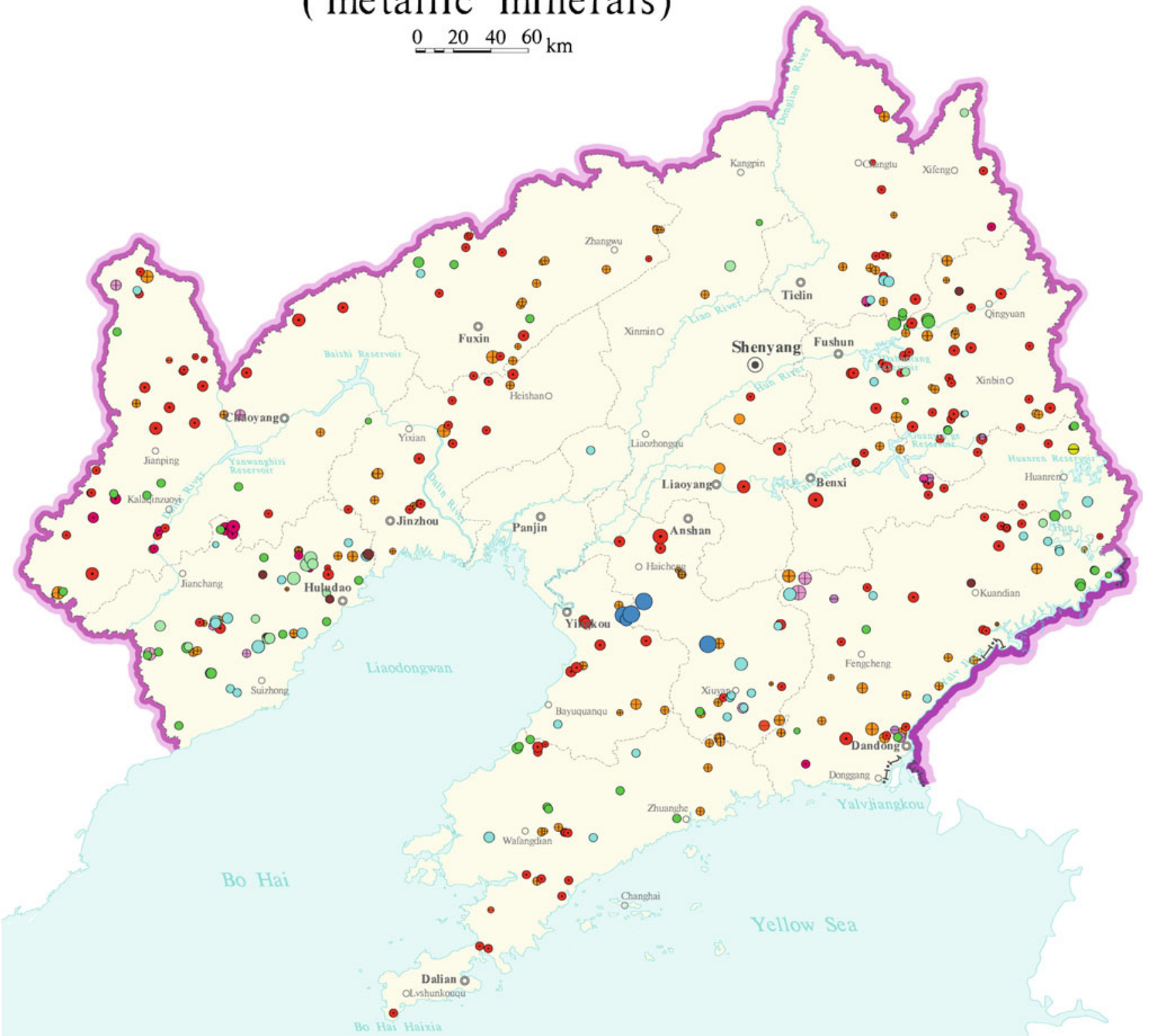




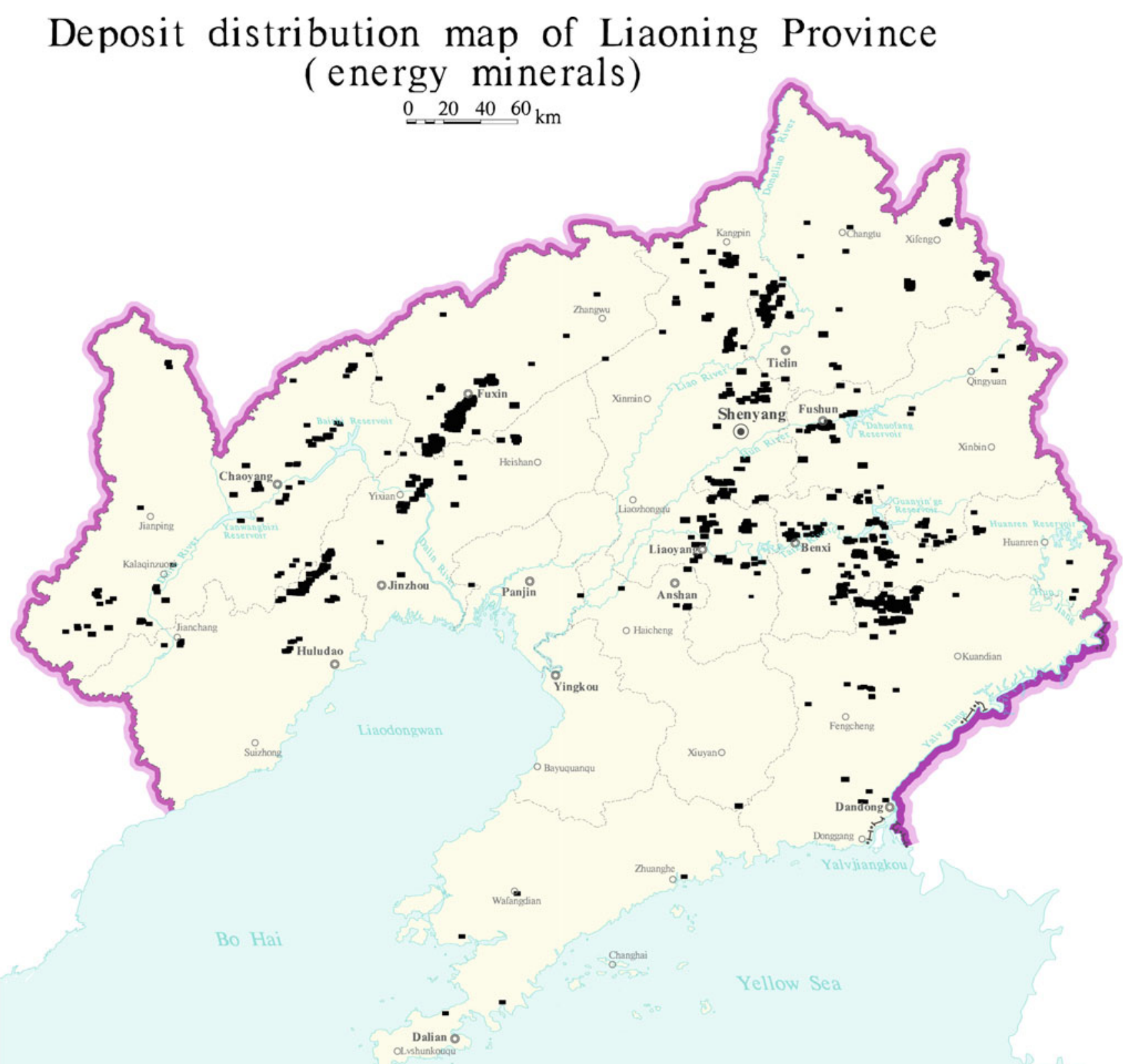




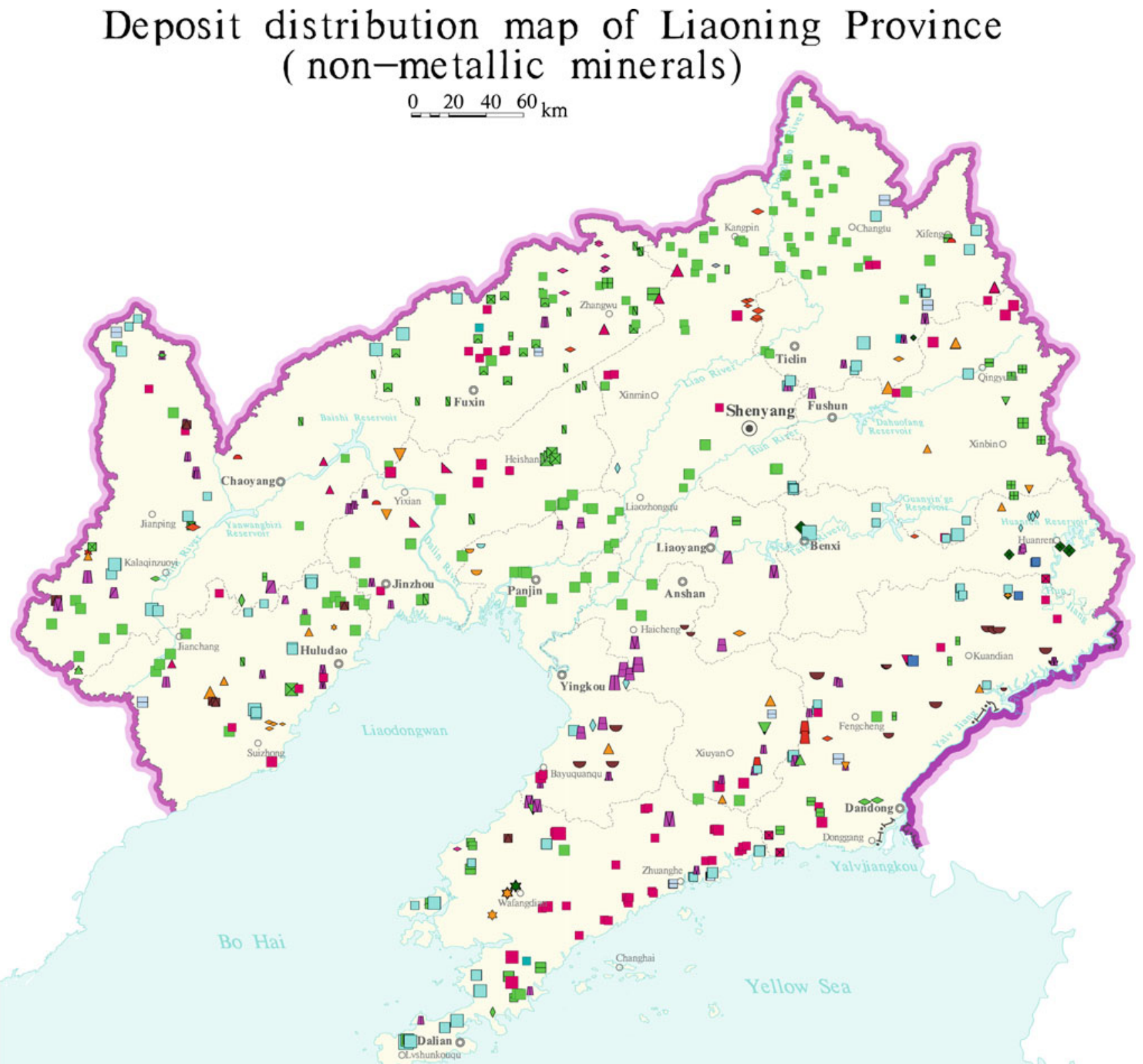




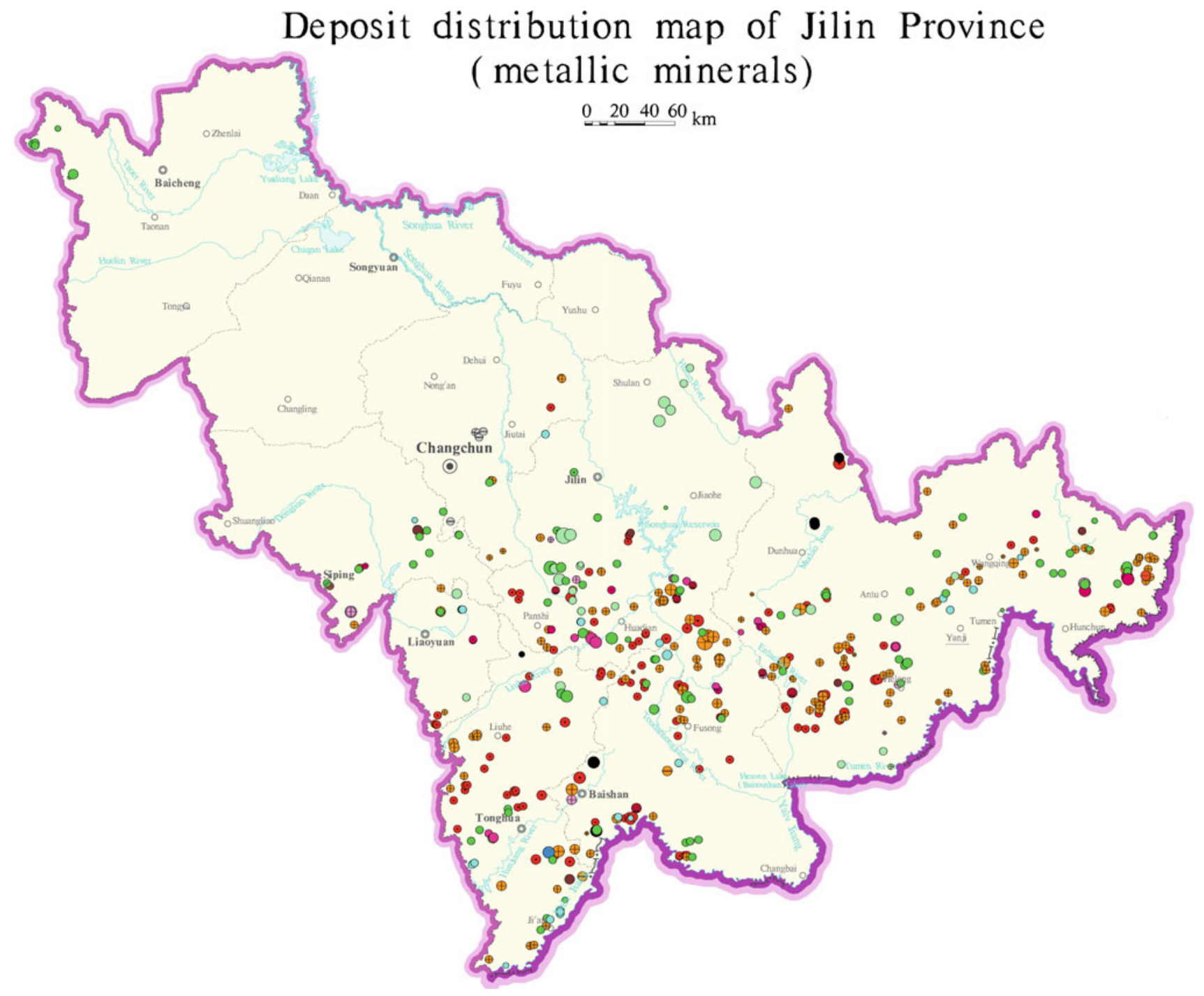


Deposit distribution map of Jilin Province

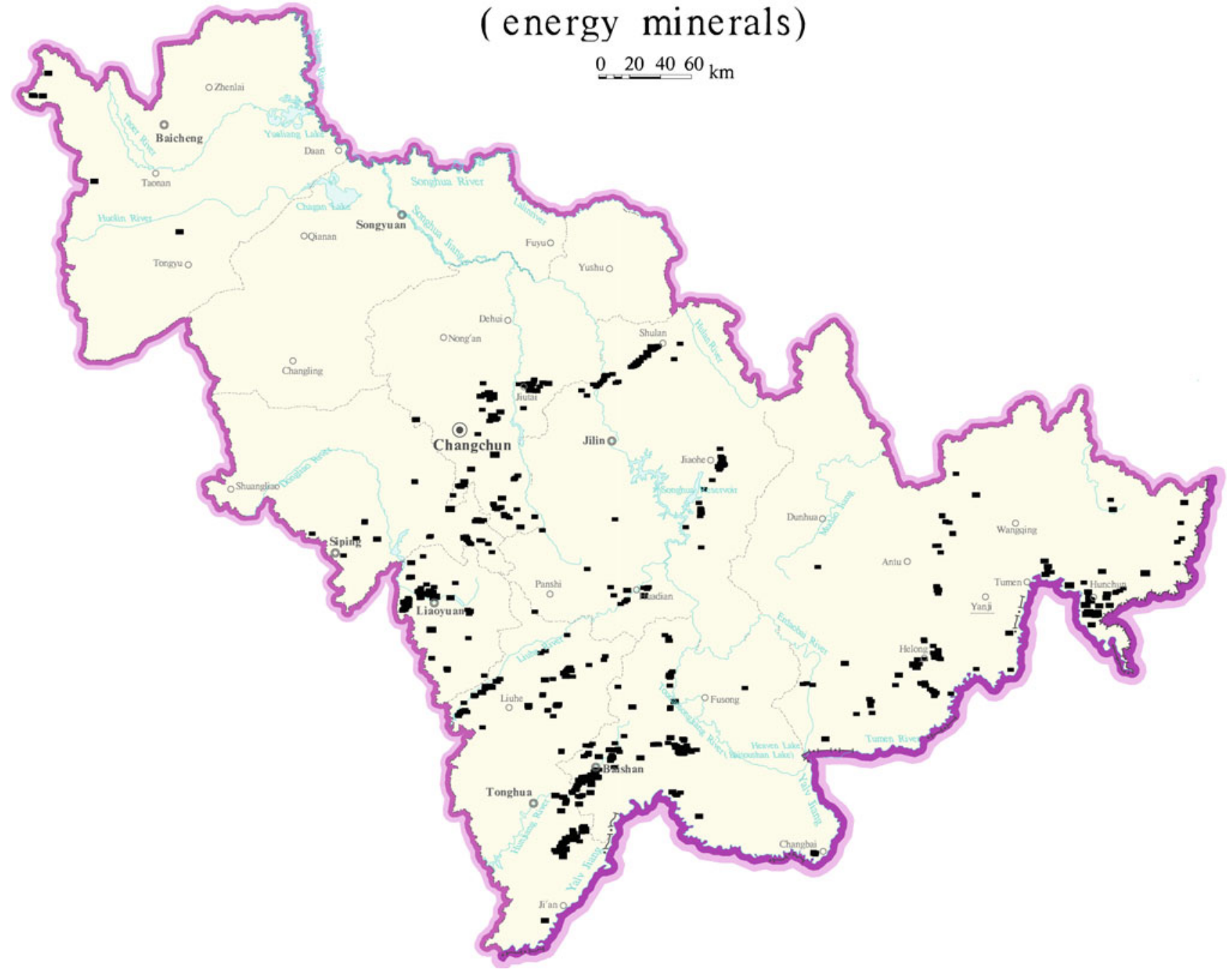




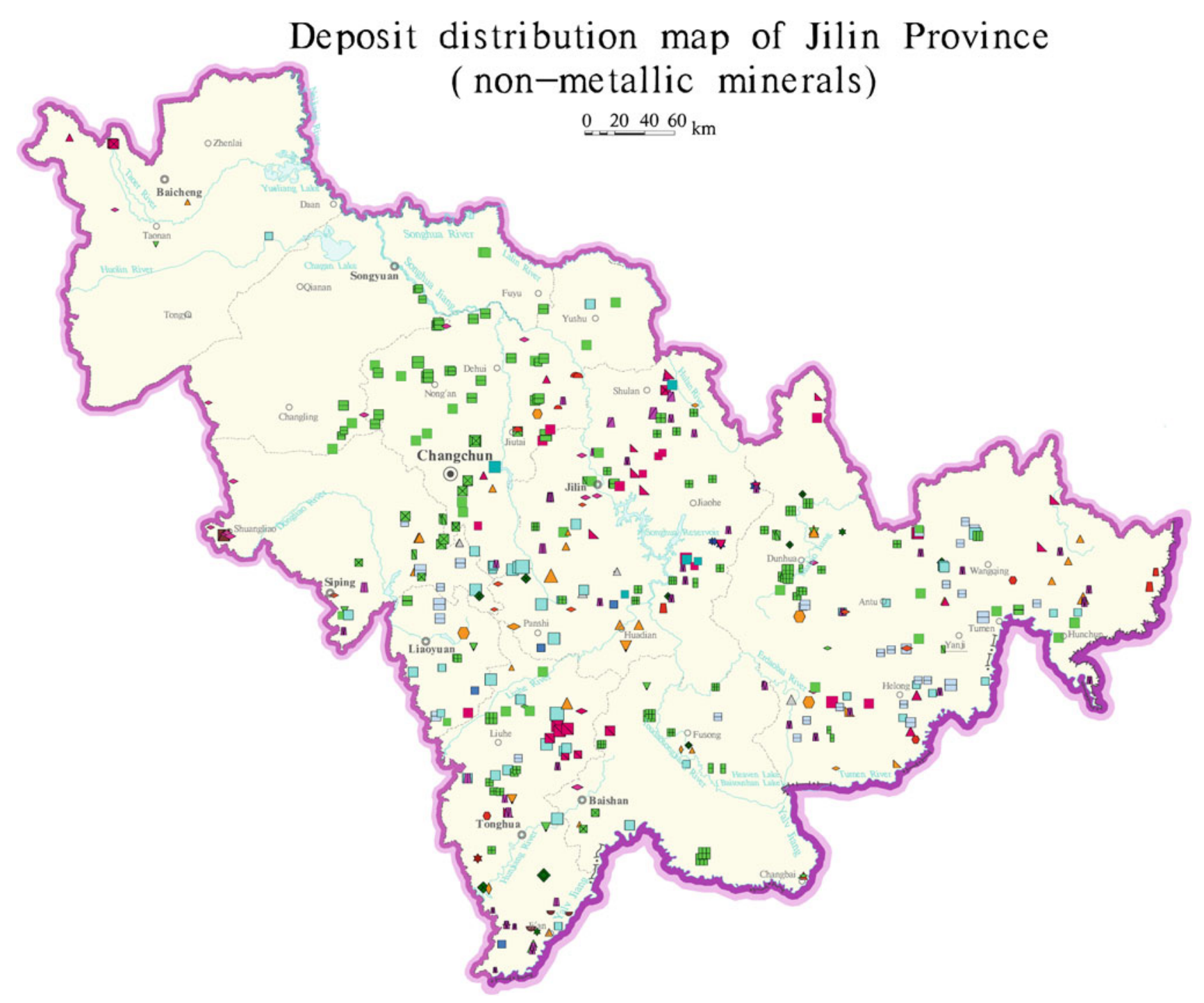




\section{Deposit distribution map of Heilongjiang Province} ( metallic minerals)

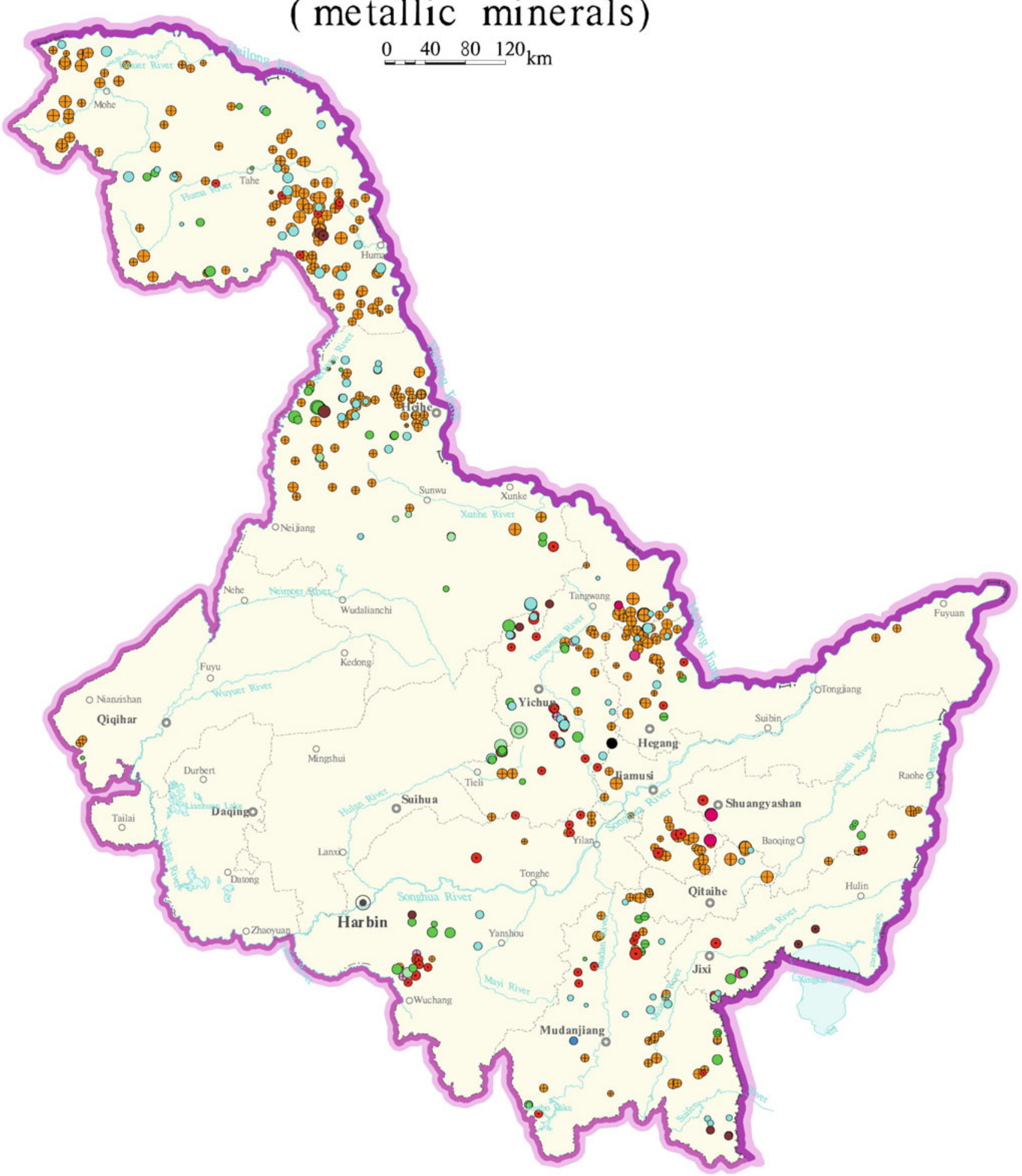




\section{Deposit distribution map of Heilongjiang Province} (energy minerals)

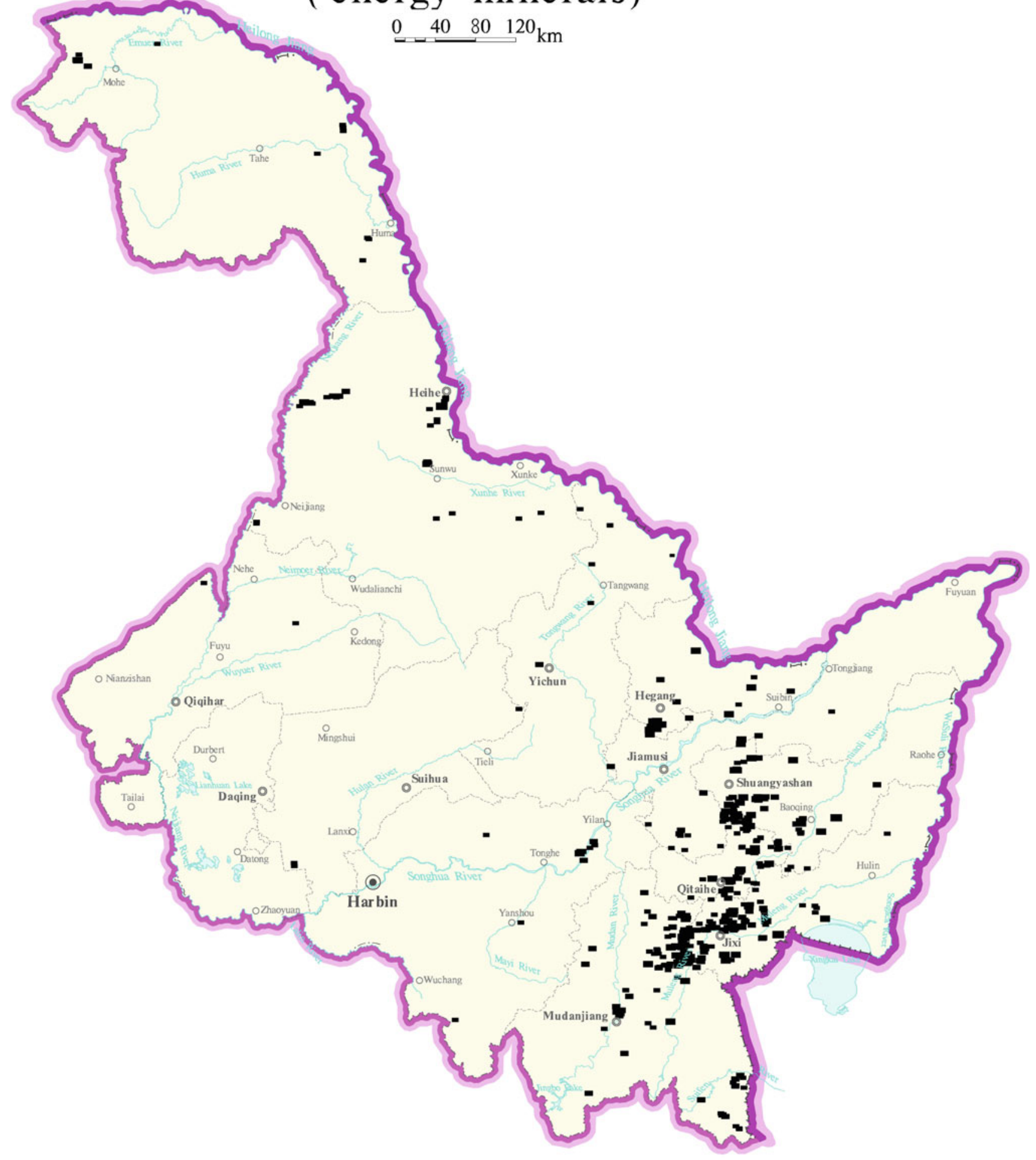




\section{Deposit distribution map of Heilongjiang Province}

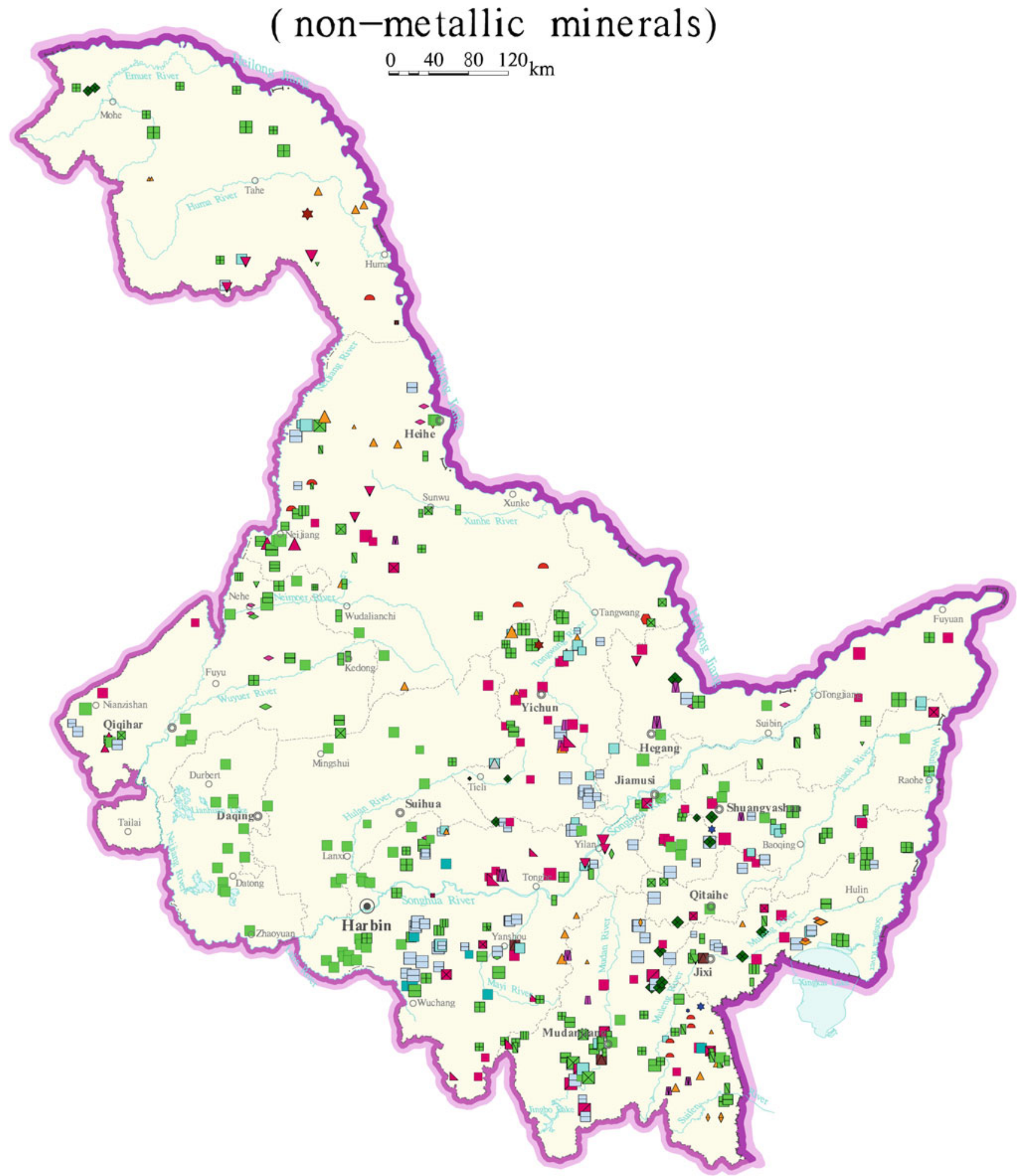




\section{Deposit distribution map of Jiangsu Province (metallic minerals)}

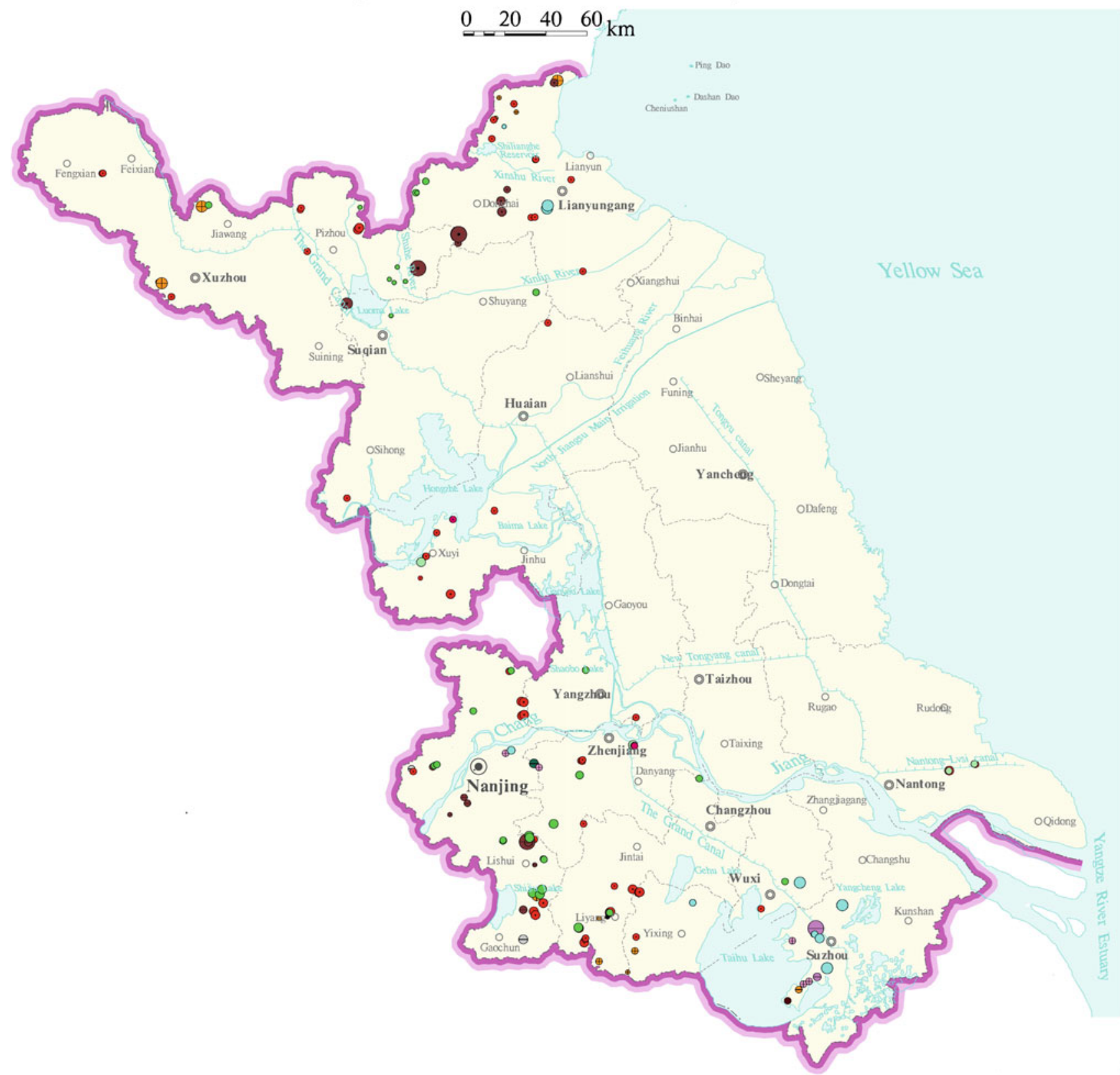


Deposit distribution map of Jiangsu Province ( energy minerals)

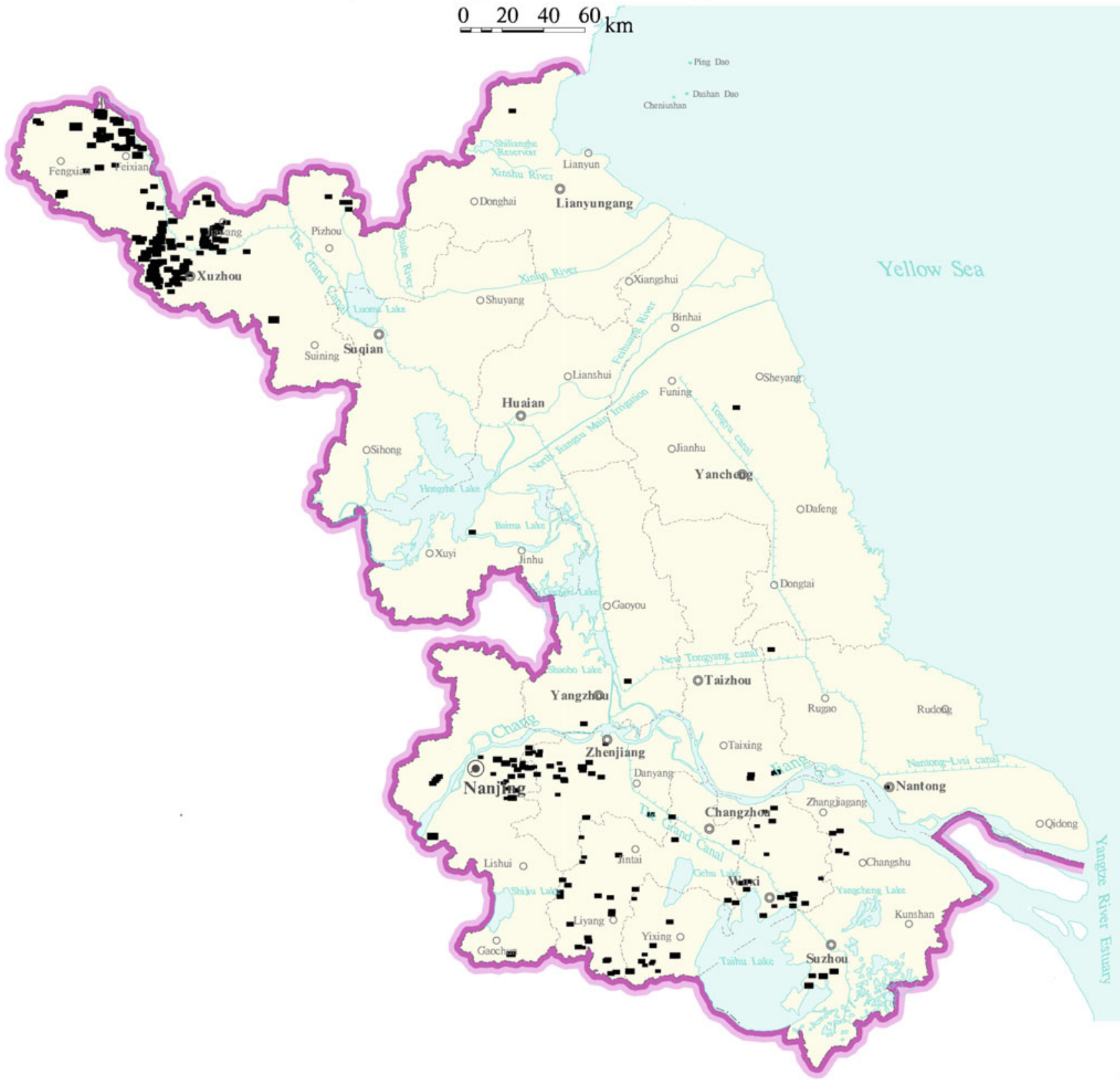




\section{Deposit distribution map of Jiangsu Province ( non-metallic minerals)}

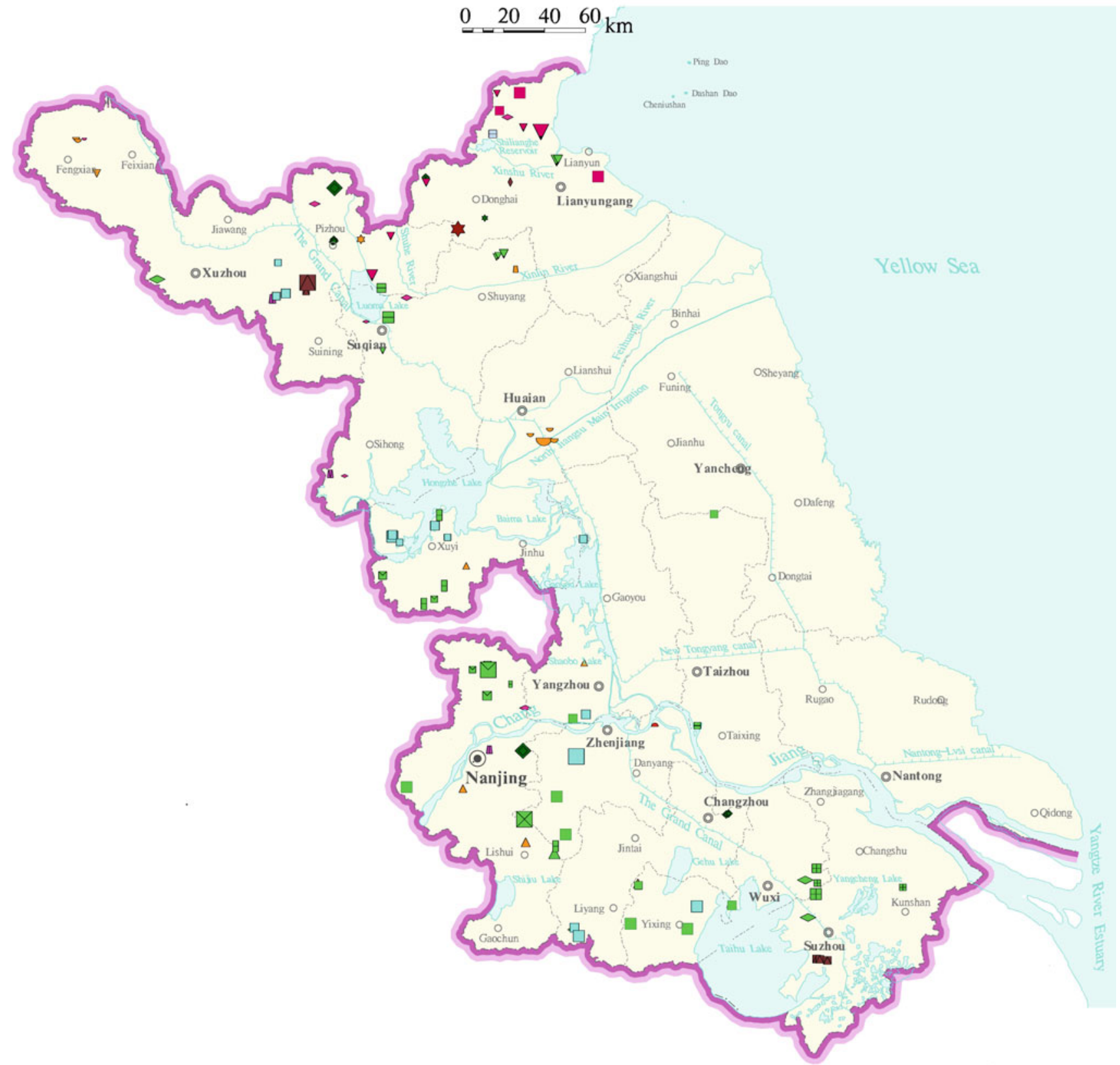




\section{Deposit distribution map of Zhejiang Province} ( metallic minerals)

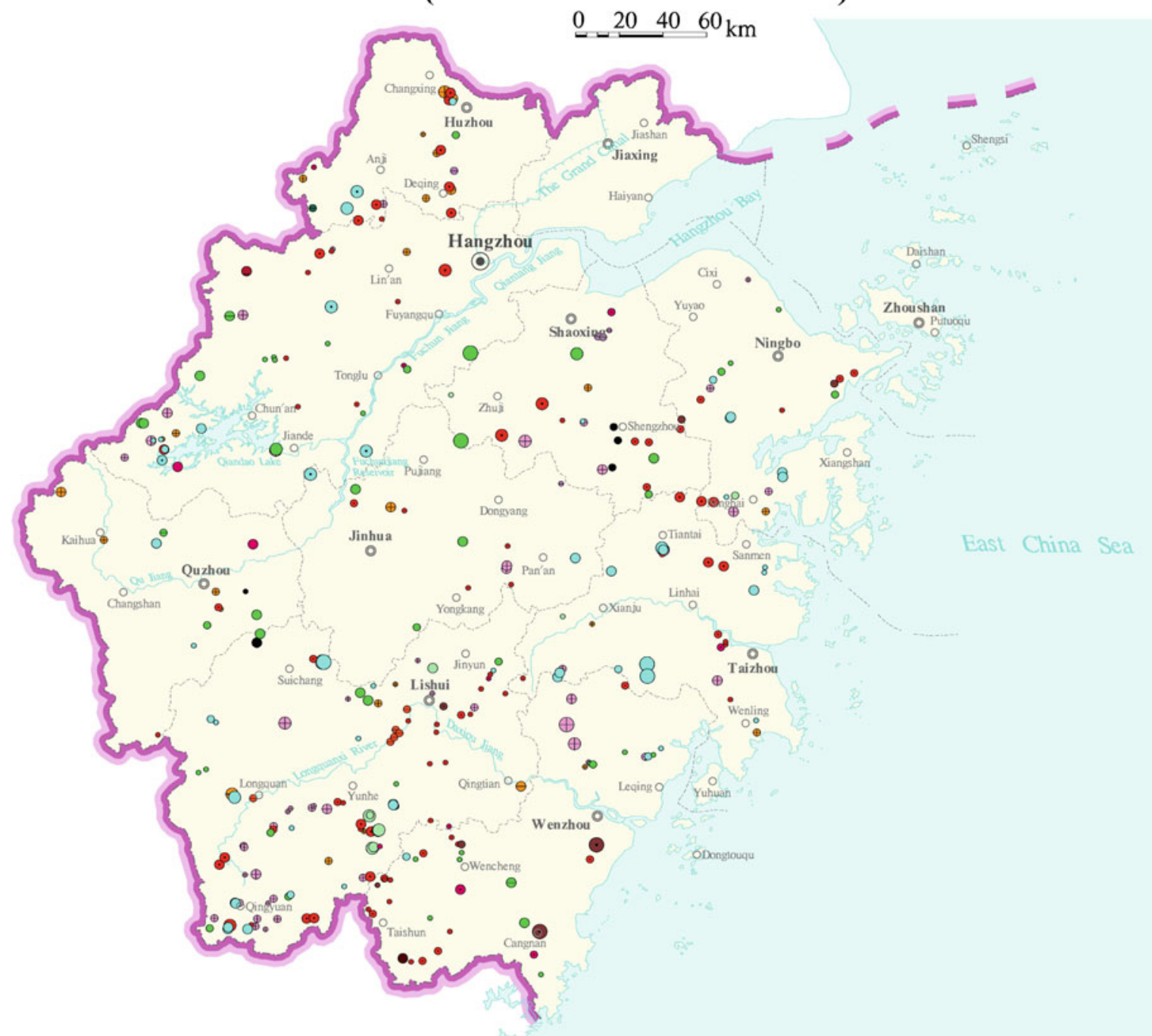


36

4 Maps of Mineral Deposits Distribution by Provinces in China

Deposit distribution map of Zhejiang Province ( energy minerals)

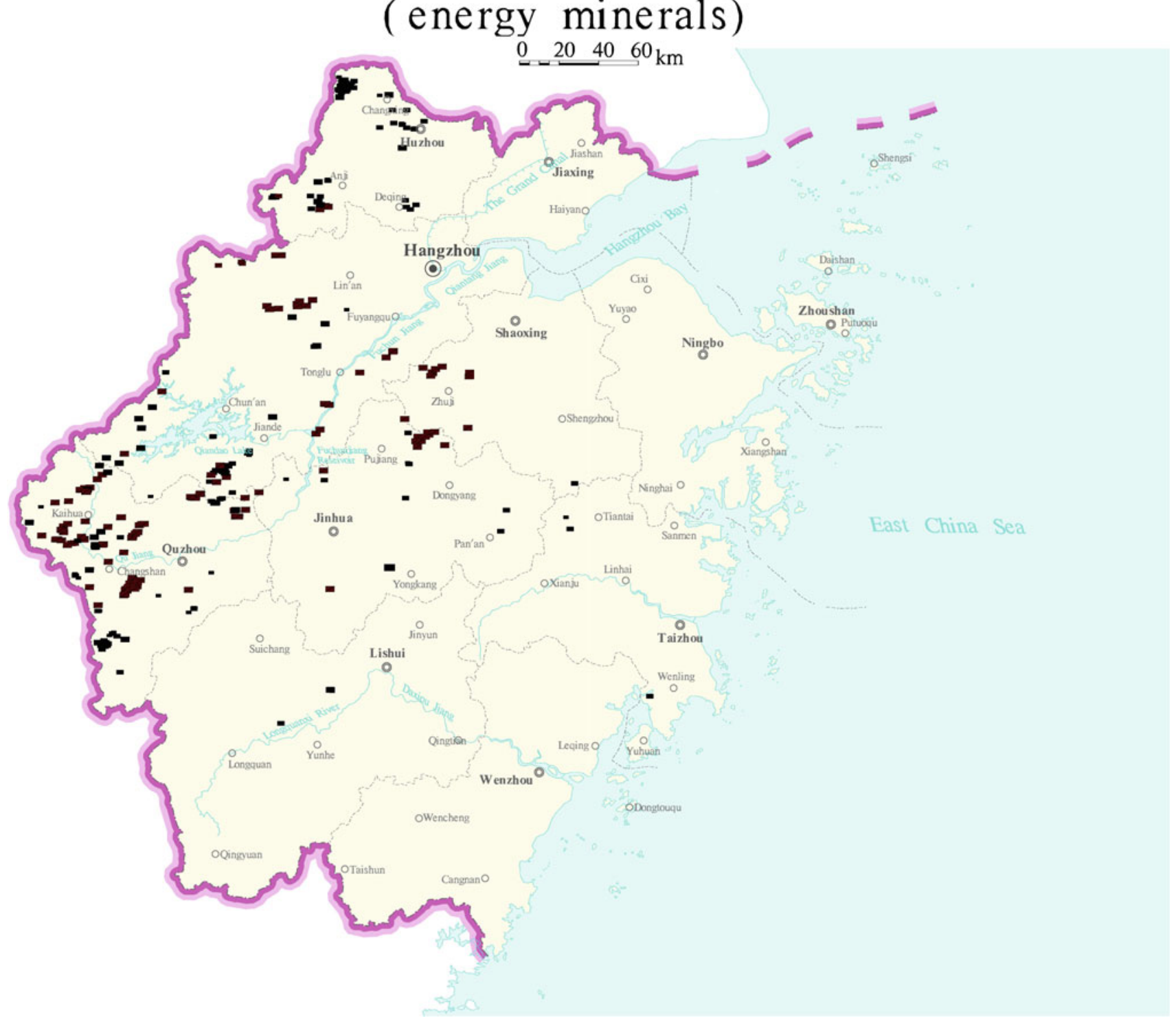


4 Maps of Mineral Deposits Distribution by Provinces in China

37

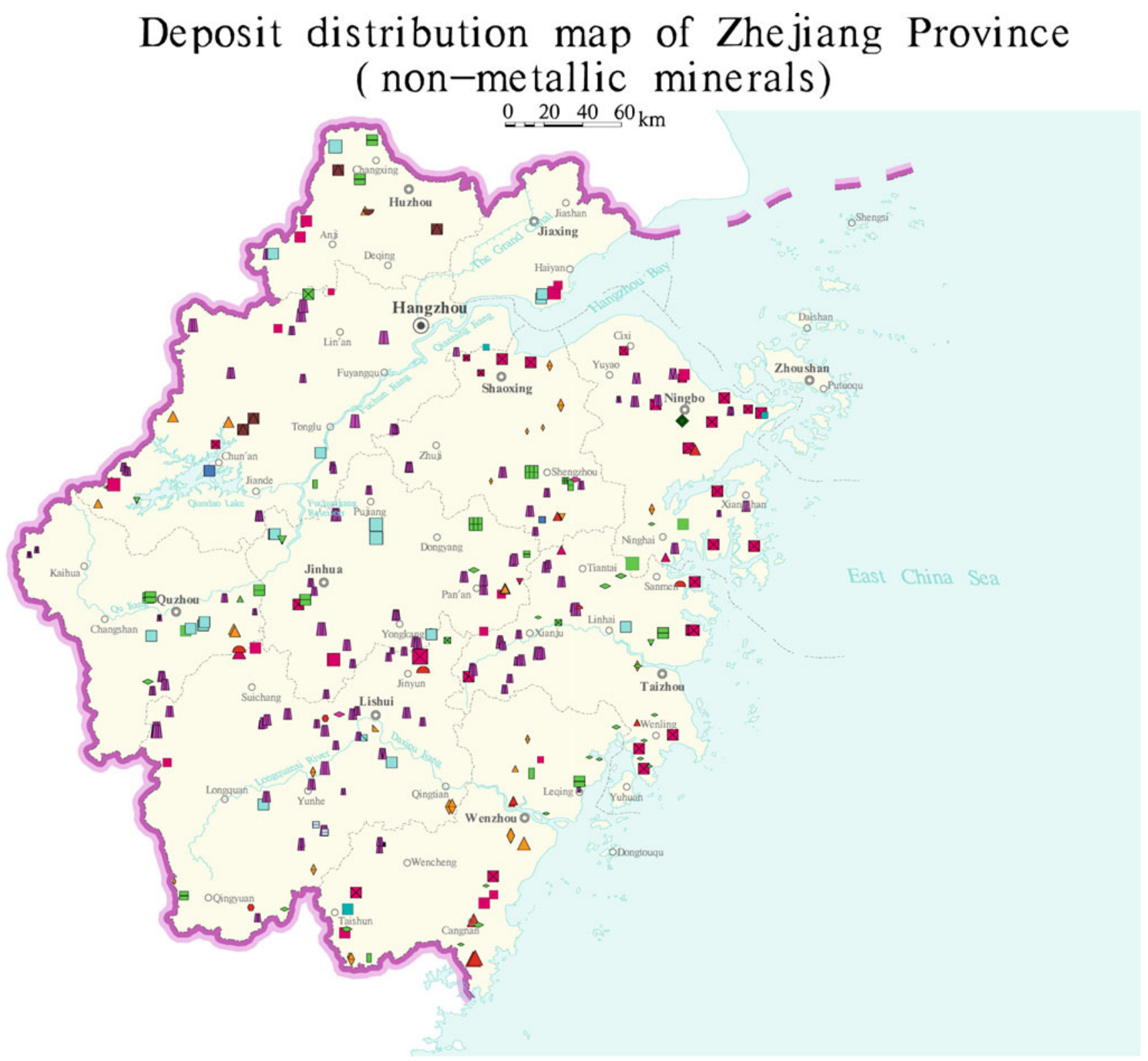


Deposit distribution map of Anhui Province

(metallic minerals)

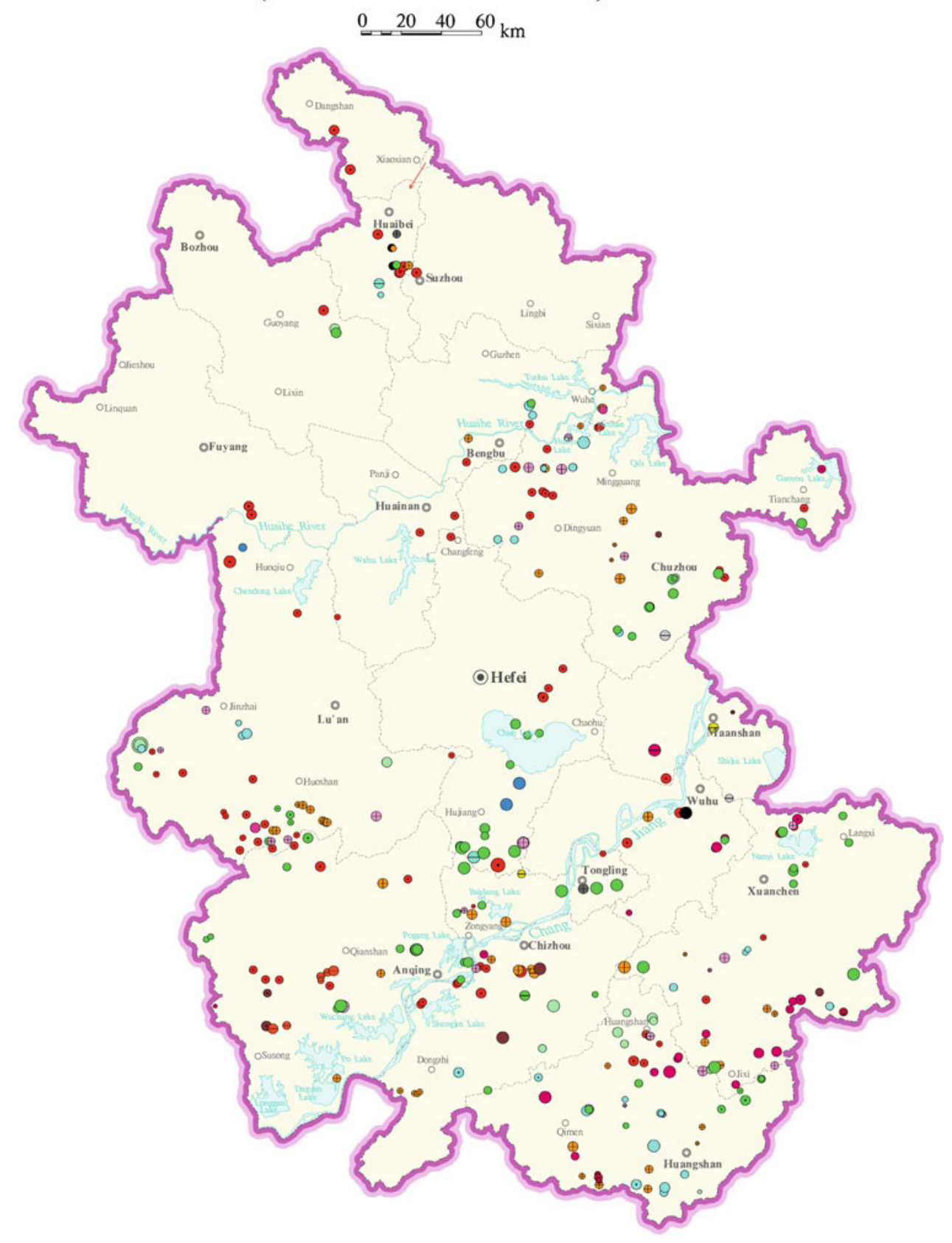


Deposit distribution map of Anhui Province ( energy minerals)

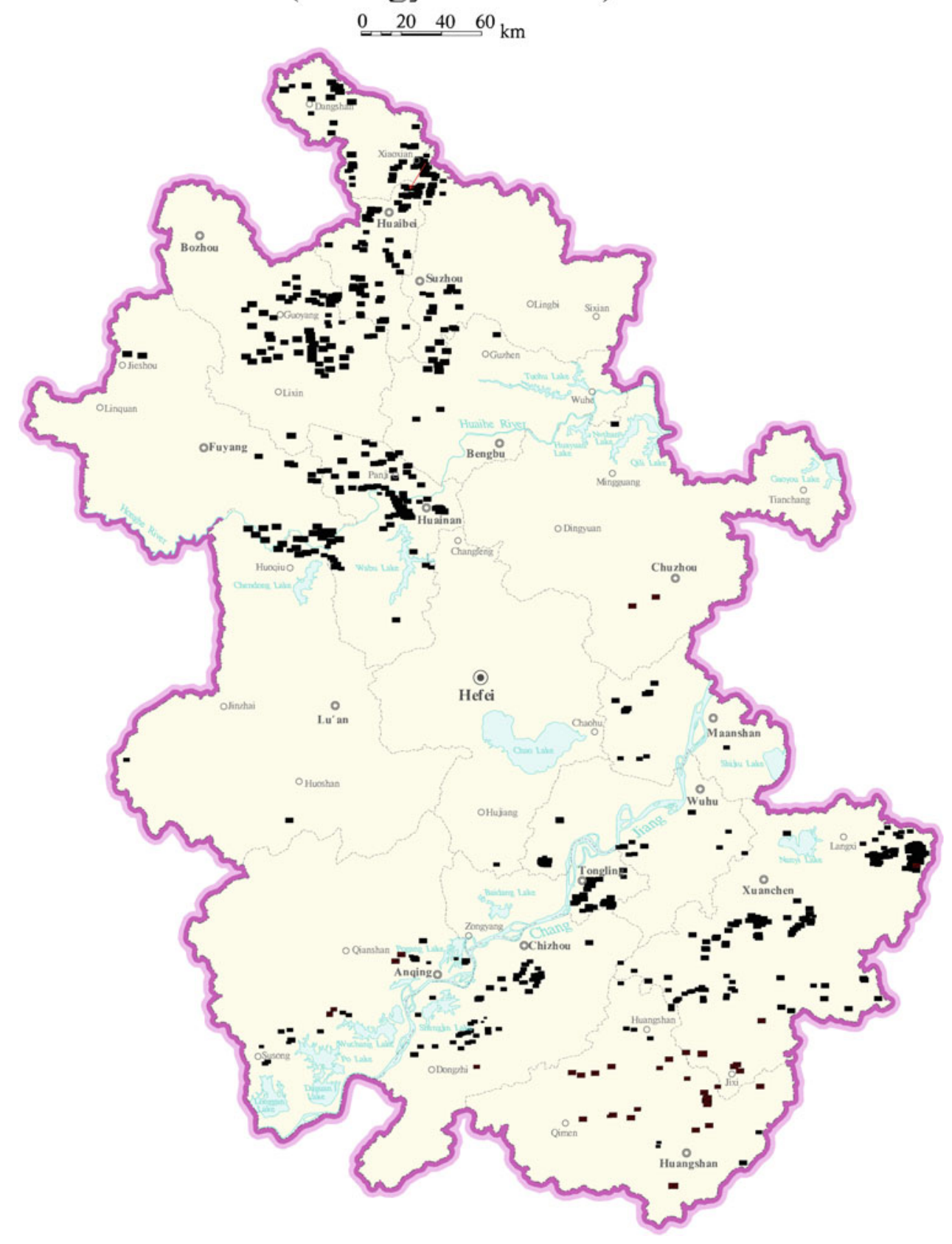




\section{Deposit distribution map of Anhui Province}

(non-metallic minerals)

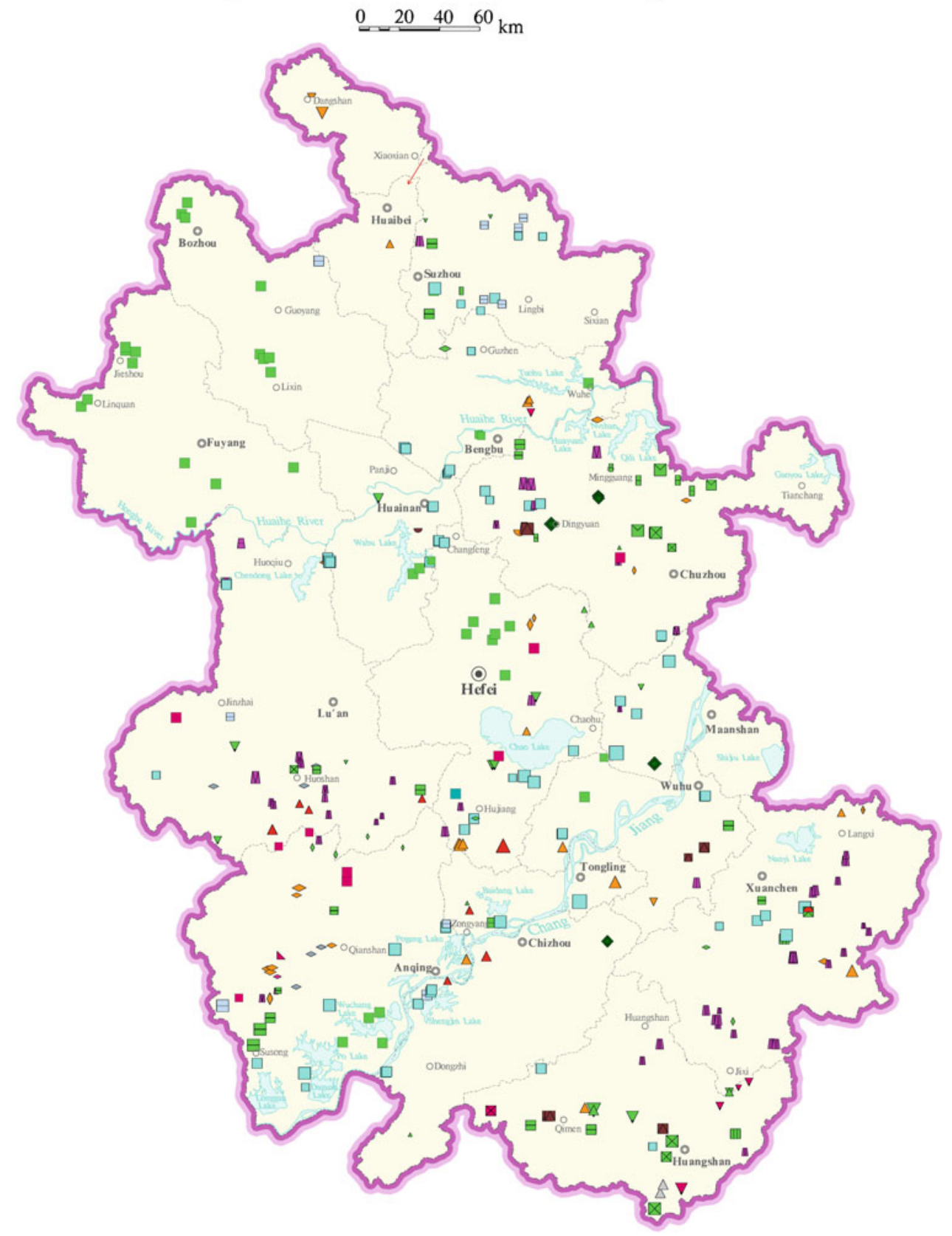


Deposit distribution map of Fujian Province

( metallic minerals)

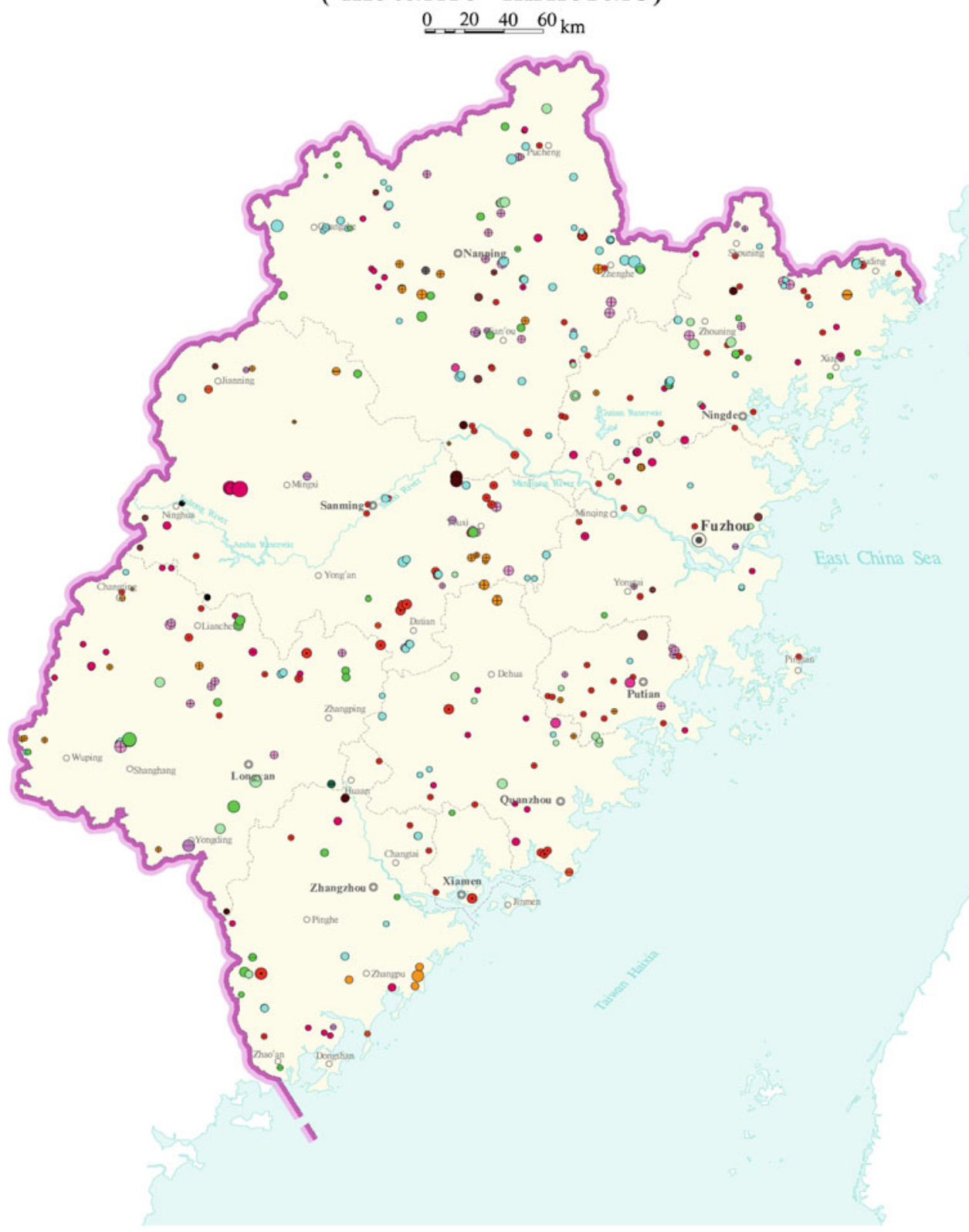




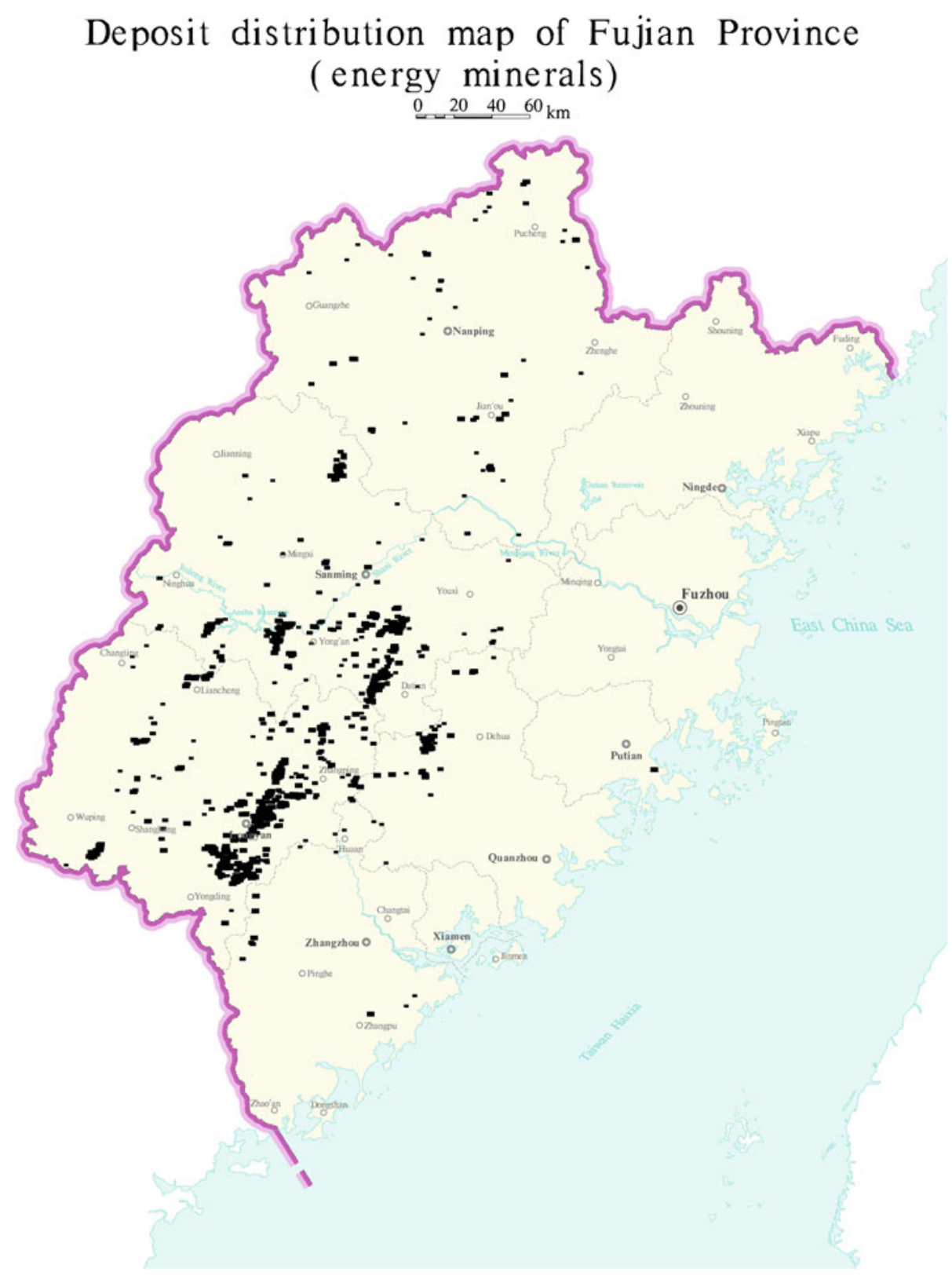




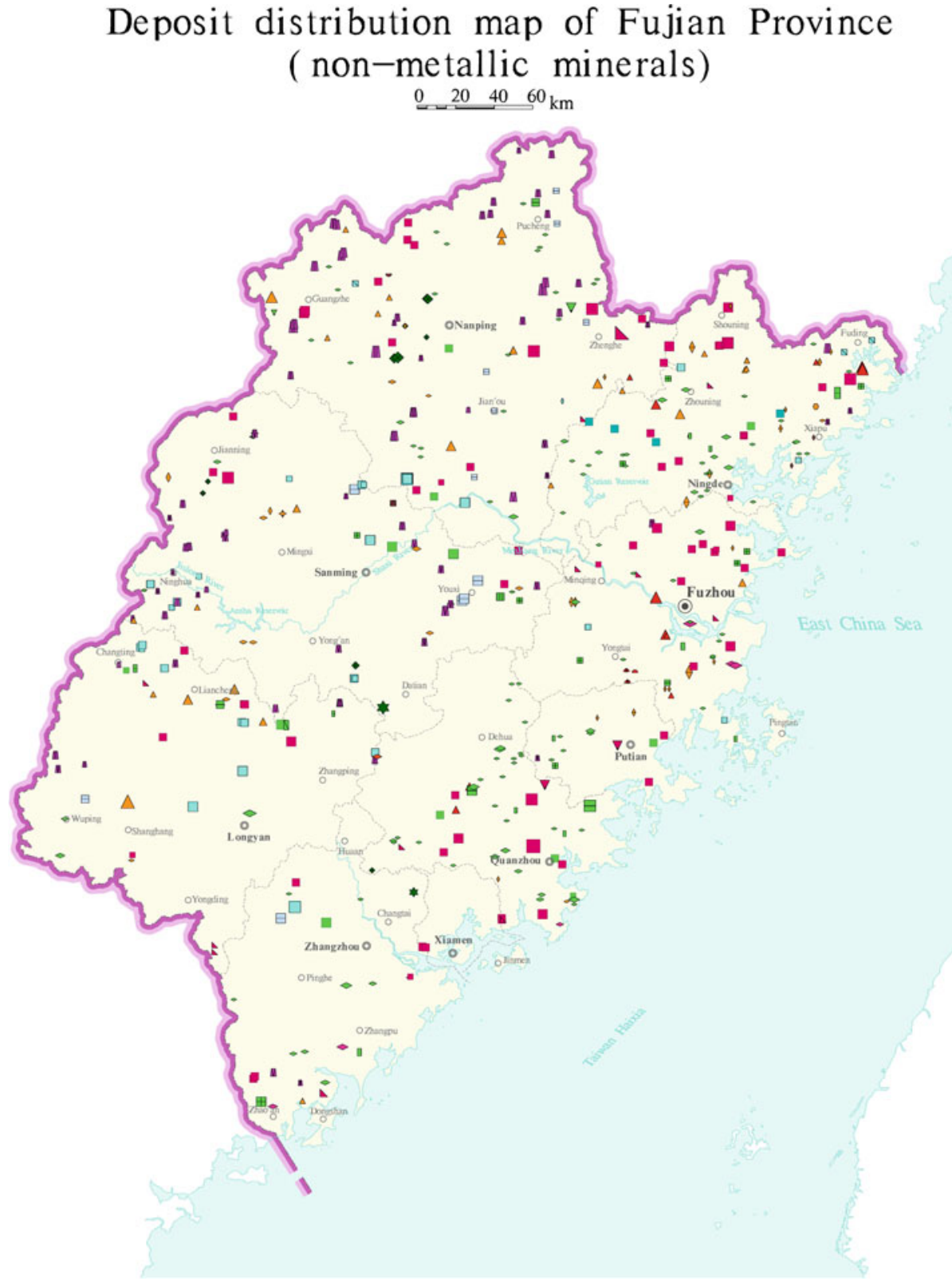




\section{Deposit distribution map of Jiangxi Province ( metallic minerals)}

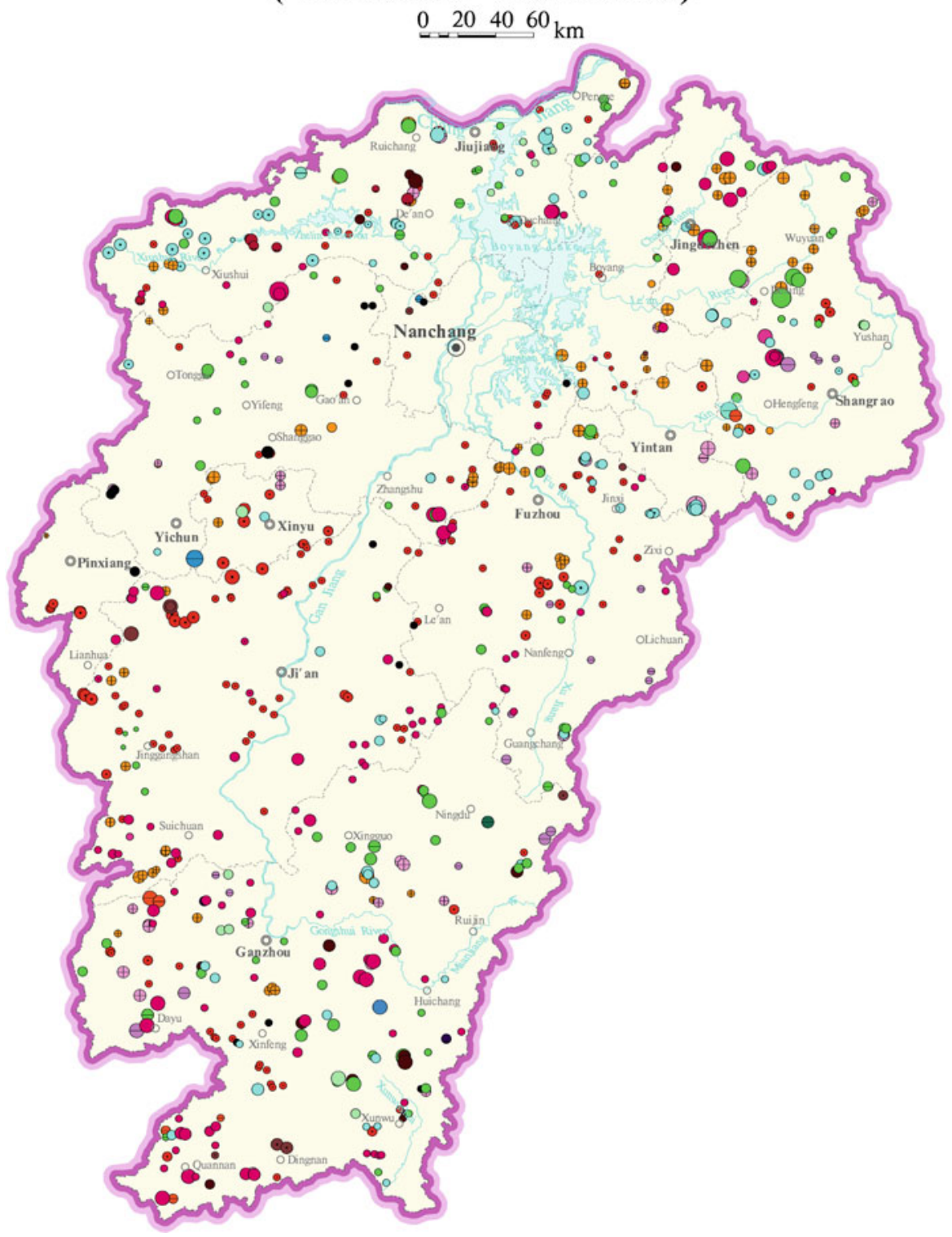


Deposit distribution map of Jiangxi Province ( energy minerals)

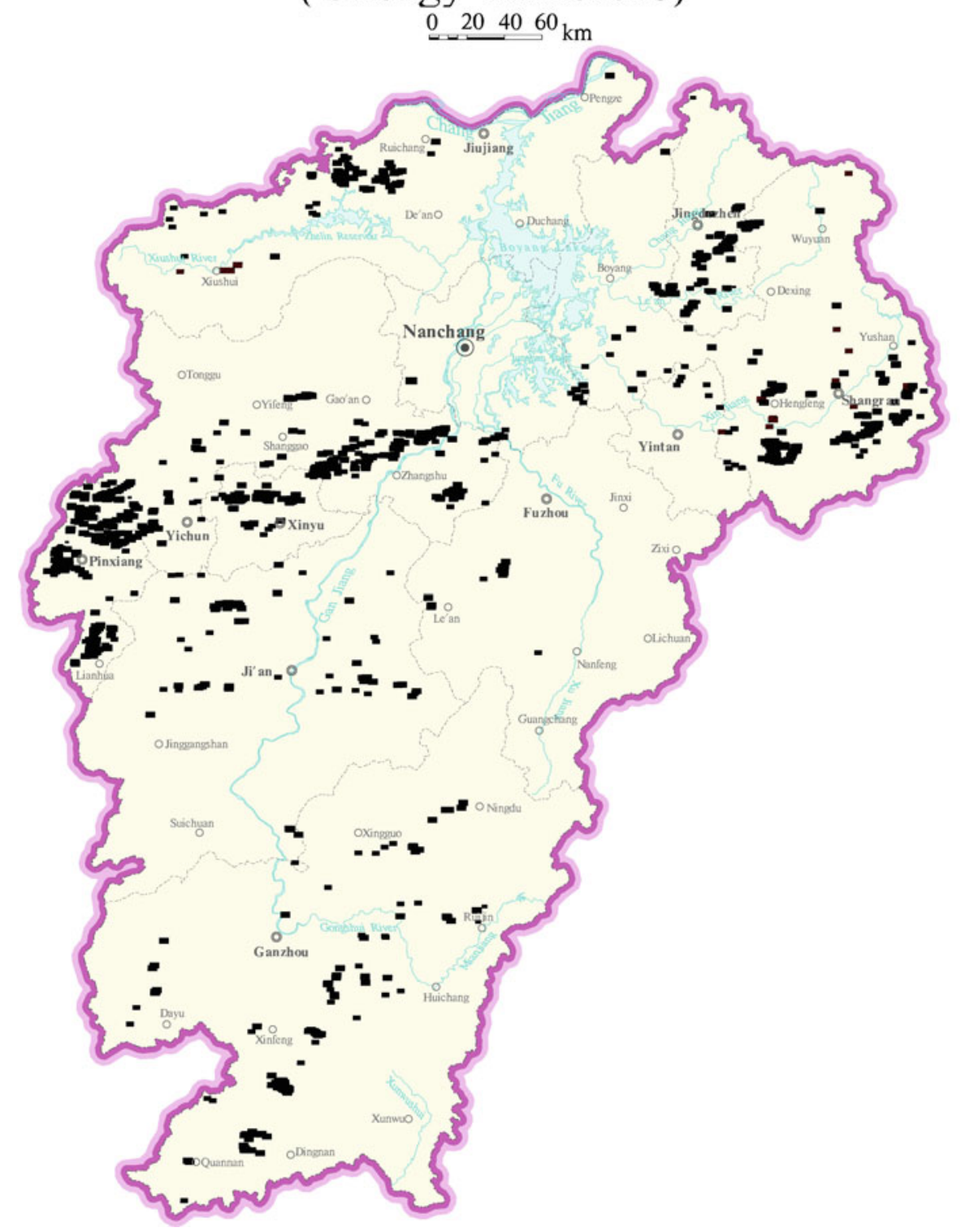




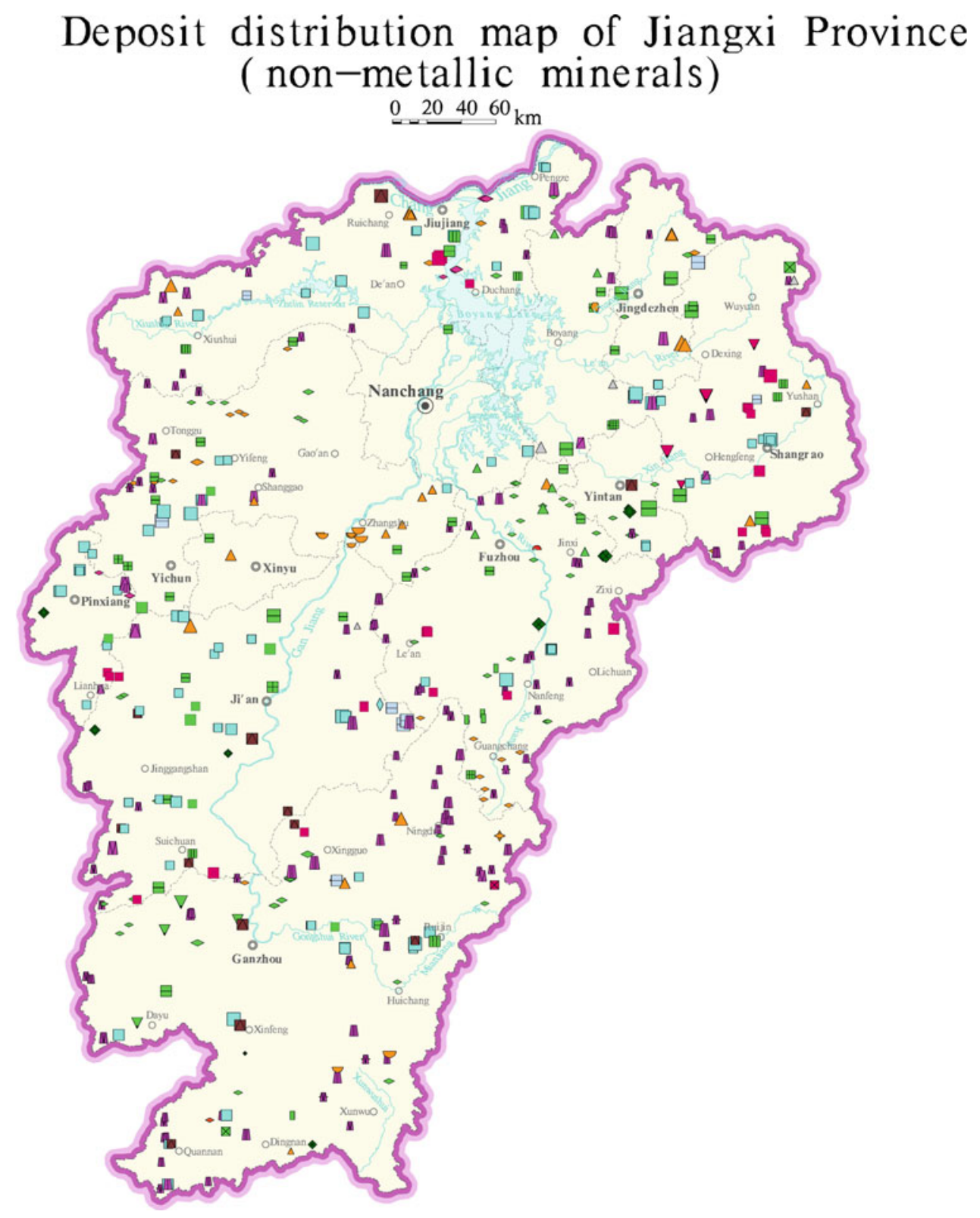


Deposit distribution map of Shandong Province (metallic minerals)

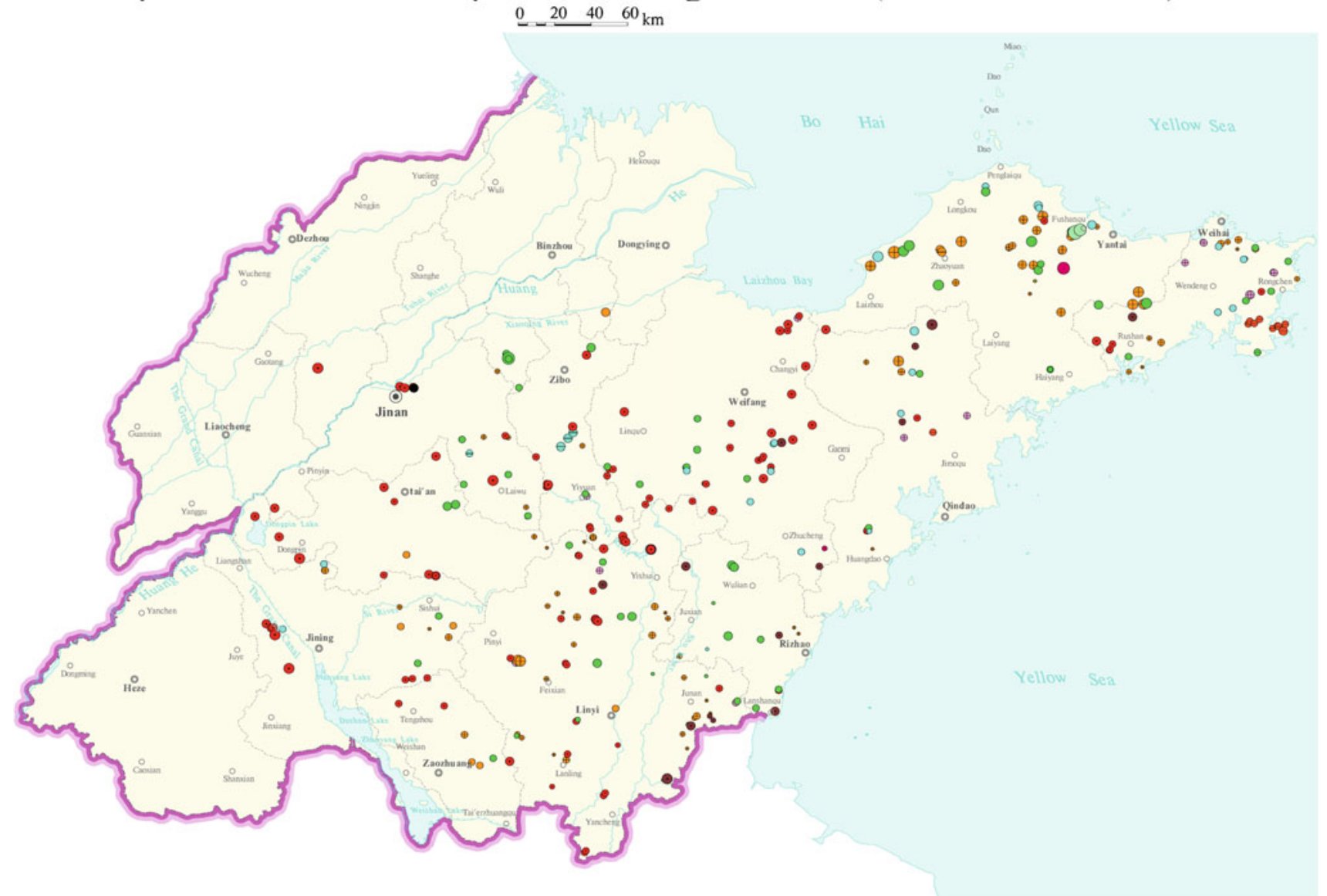


Deposit distribution map of Shandong Province (energy minerals)

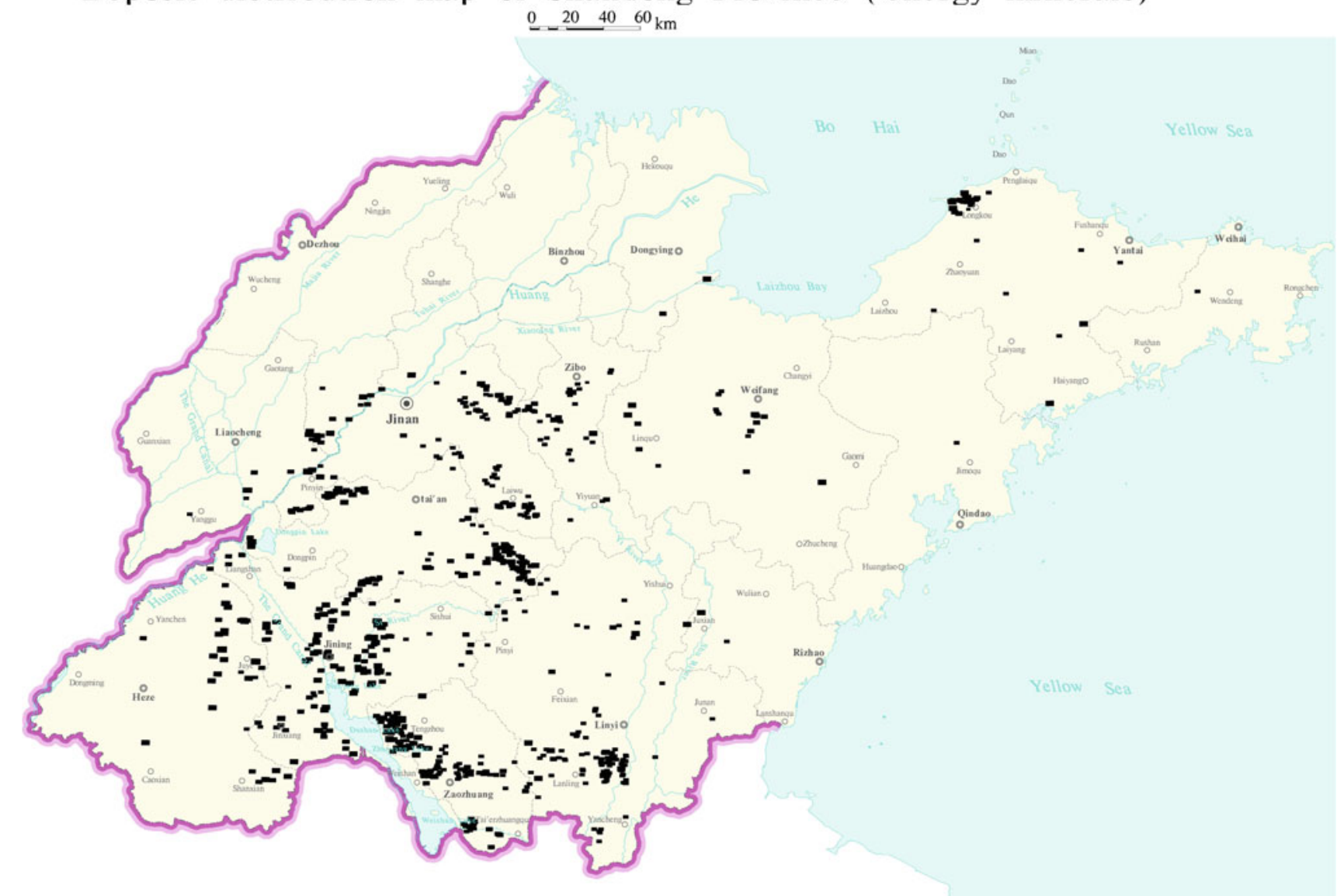


Deposit distribution map of Shandong Province (non-metallic minerals)

$$
\text { - } 20.4060 \mathrm{~km}
$$

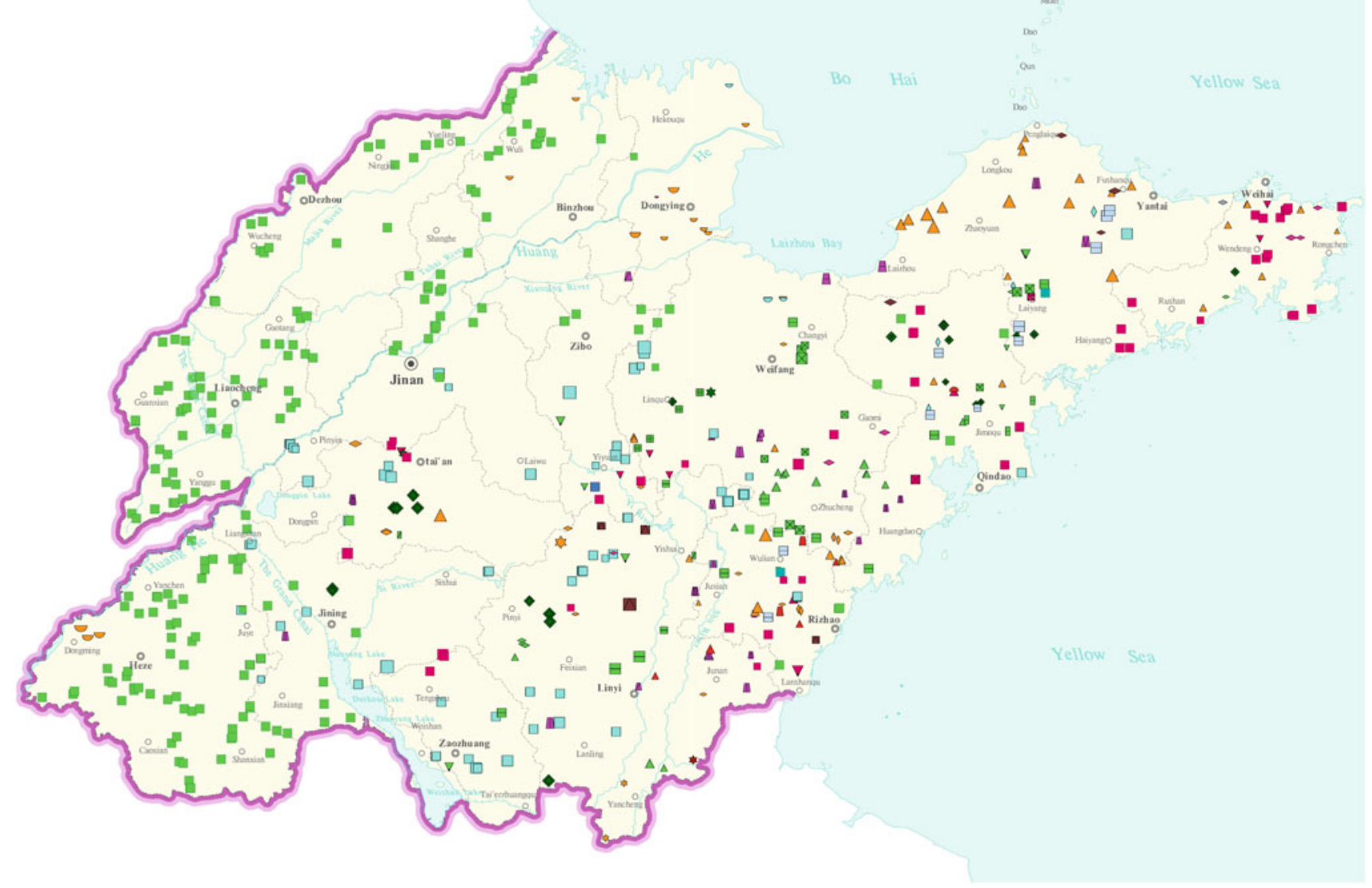




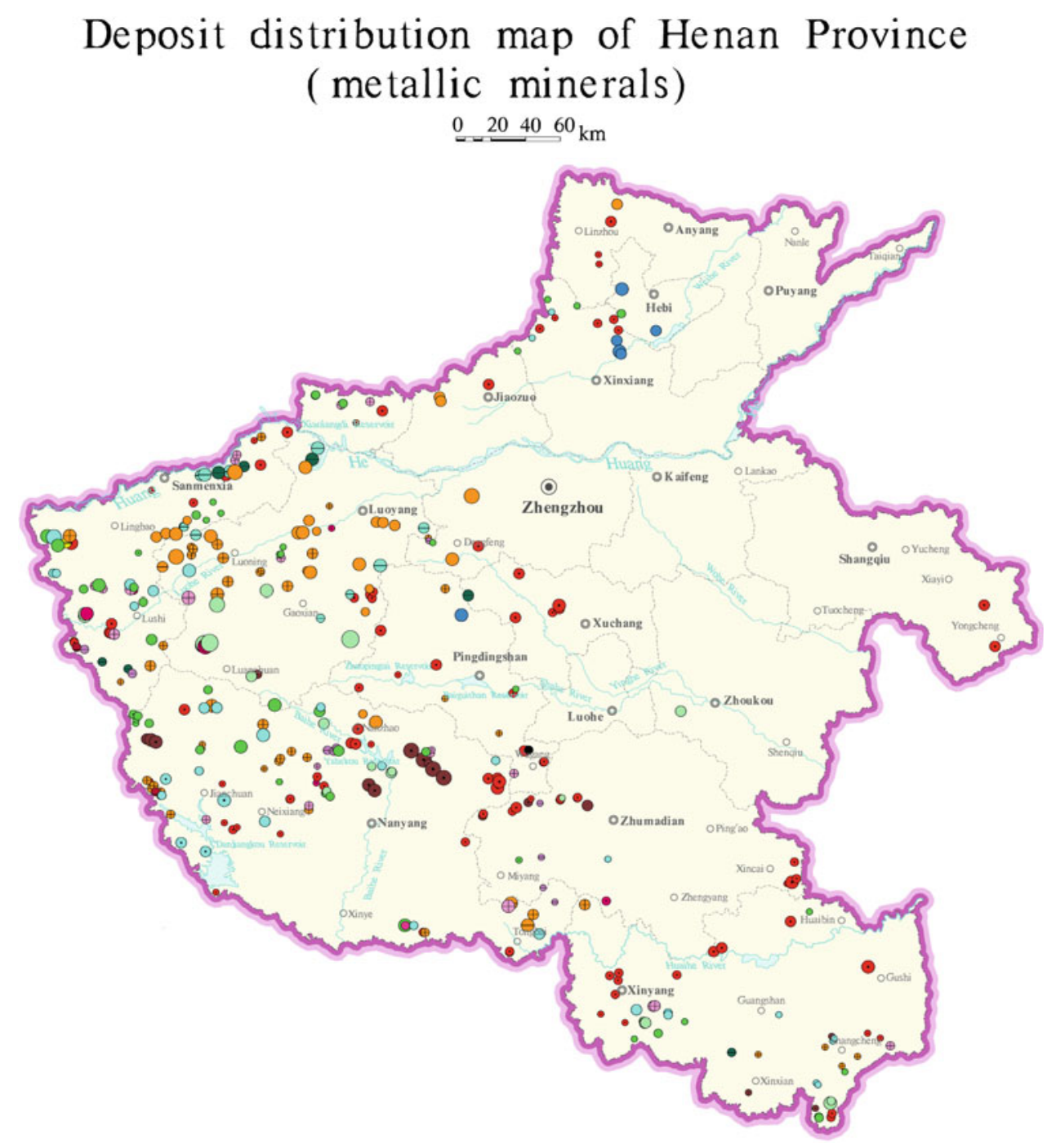




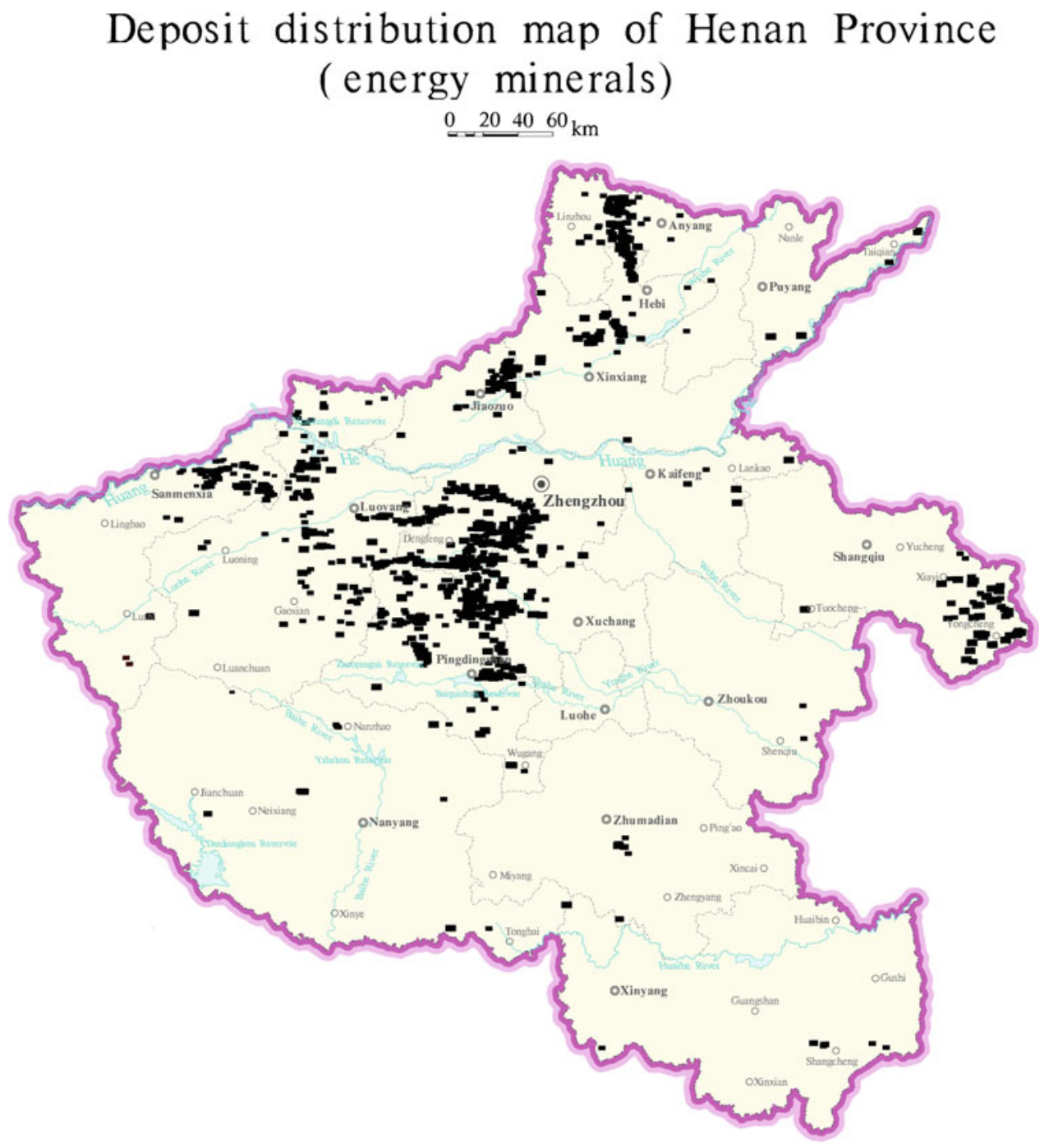




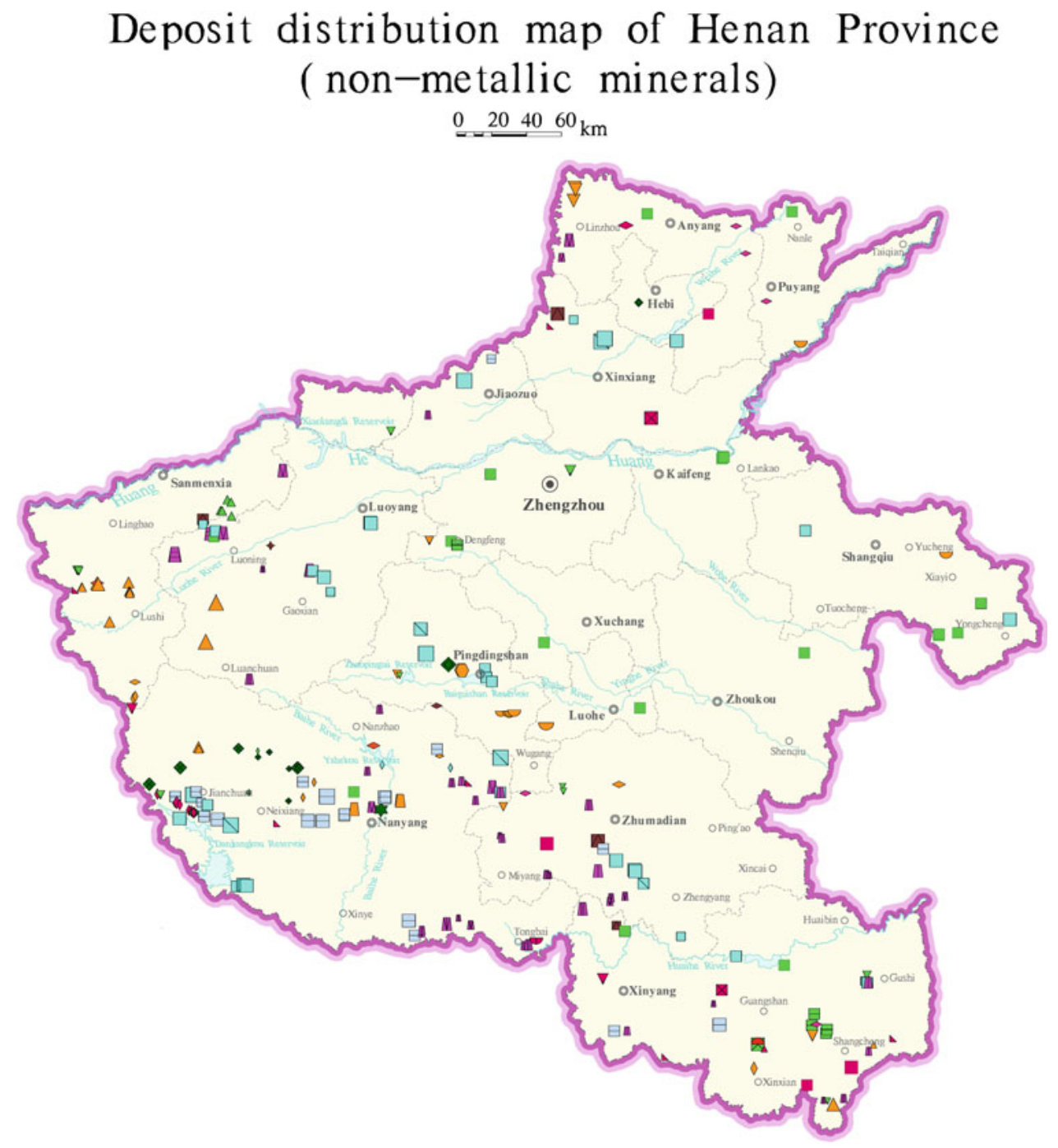




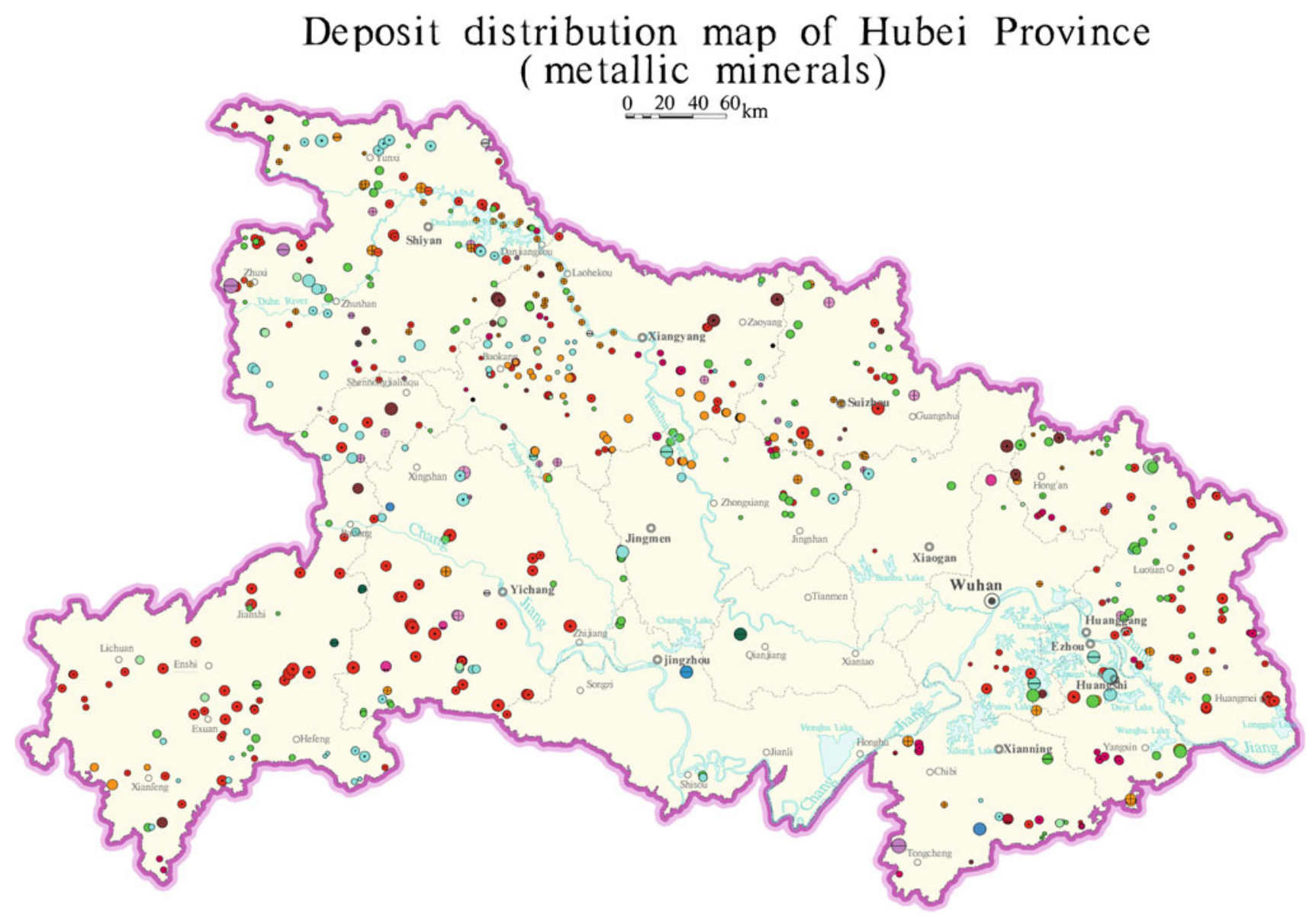




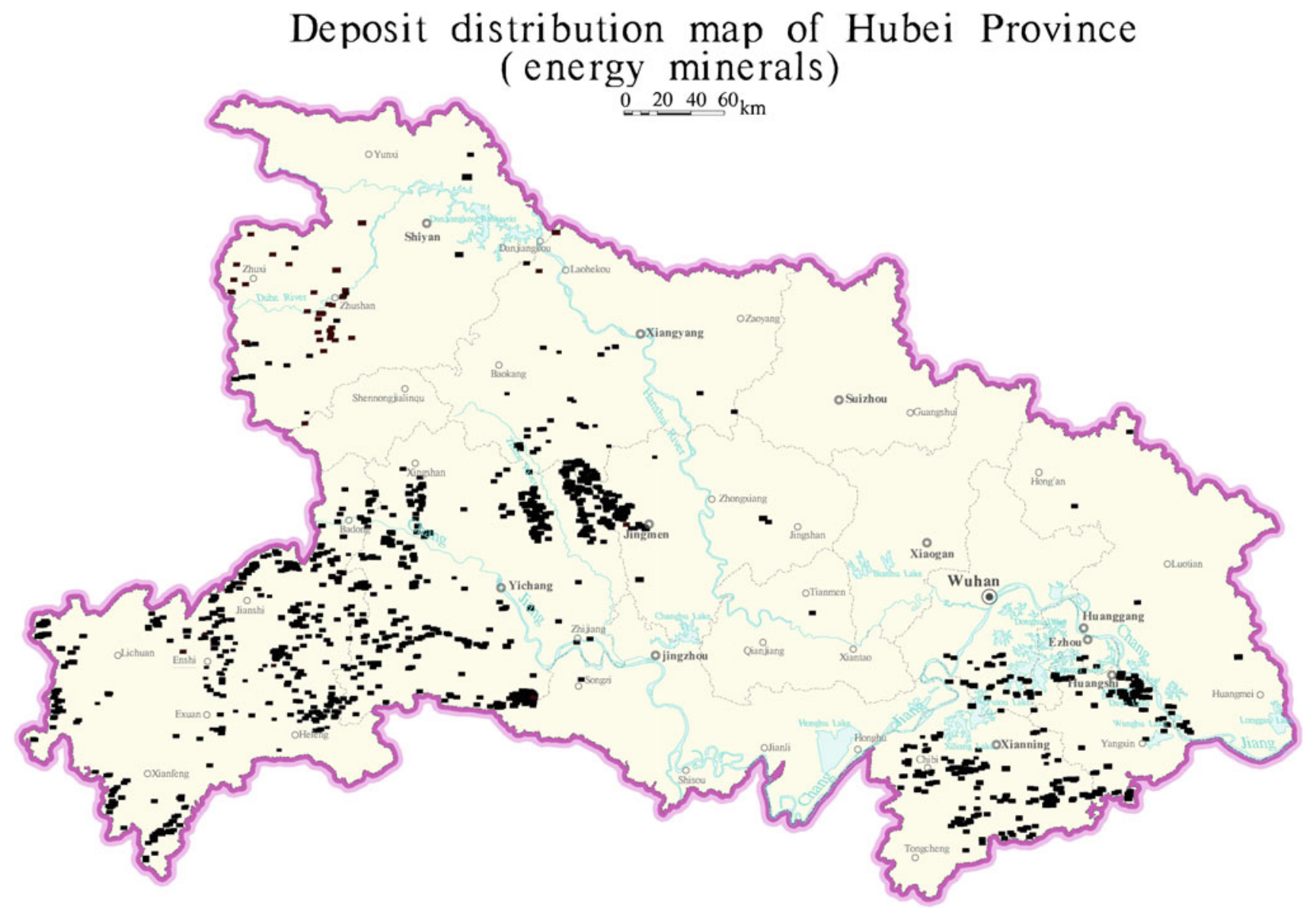




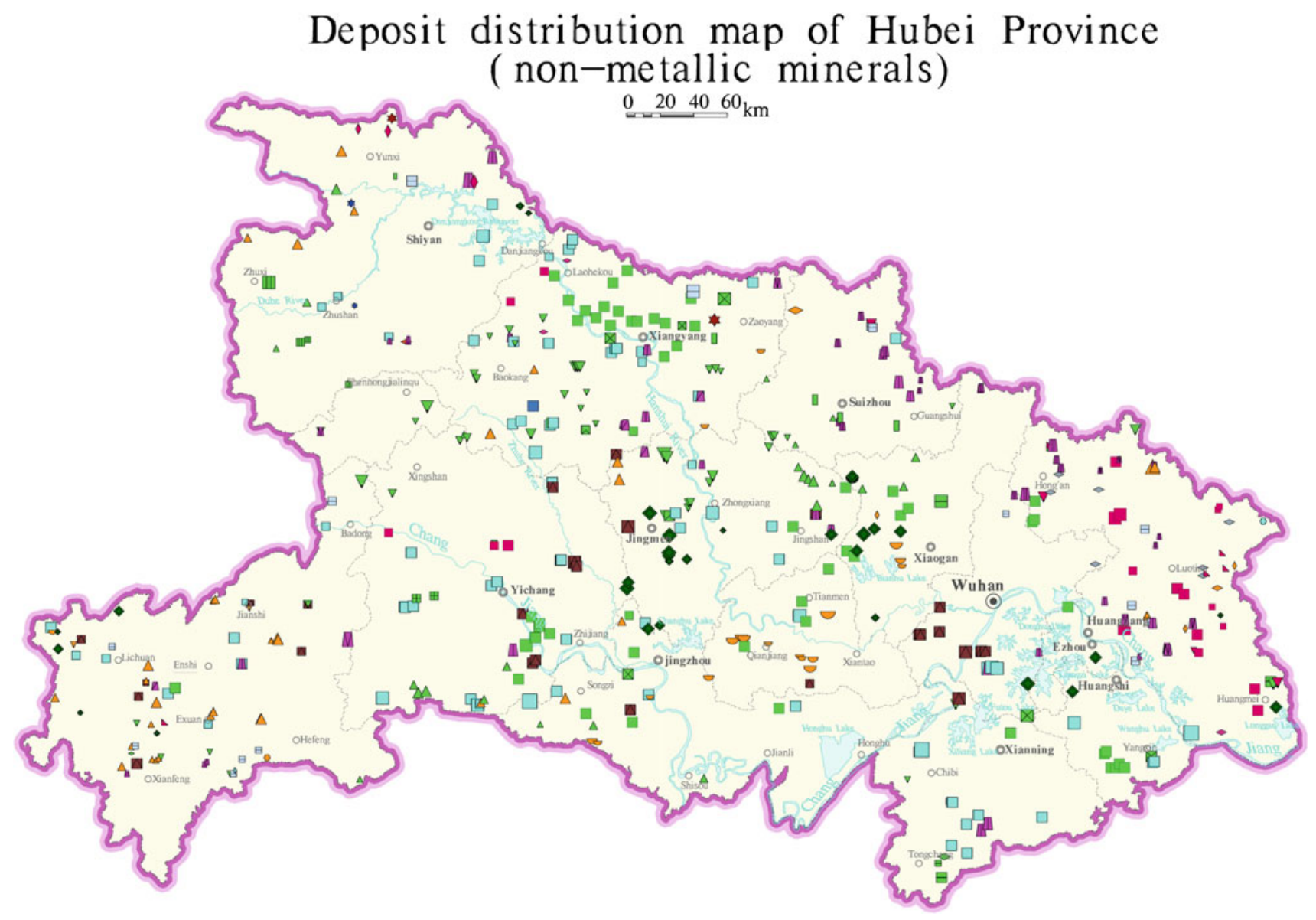




\section{Deposit distribution map of Hunan Province (metallic minerals)}

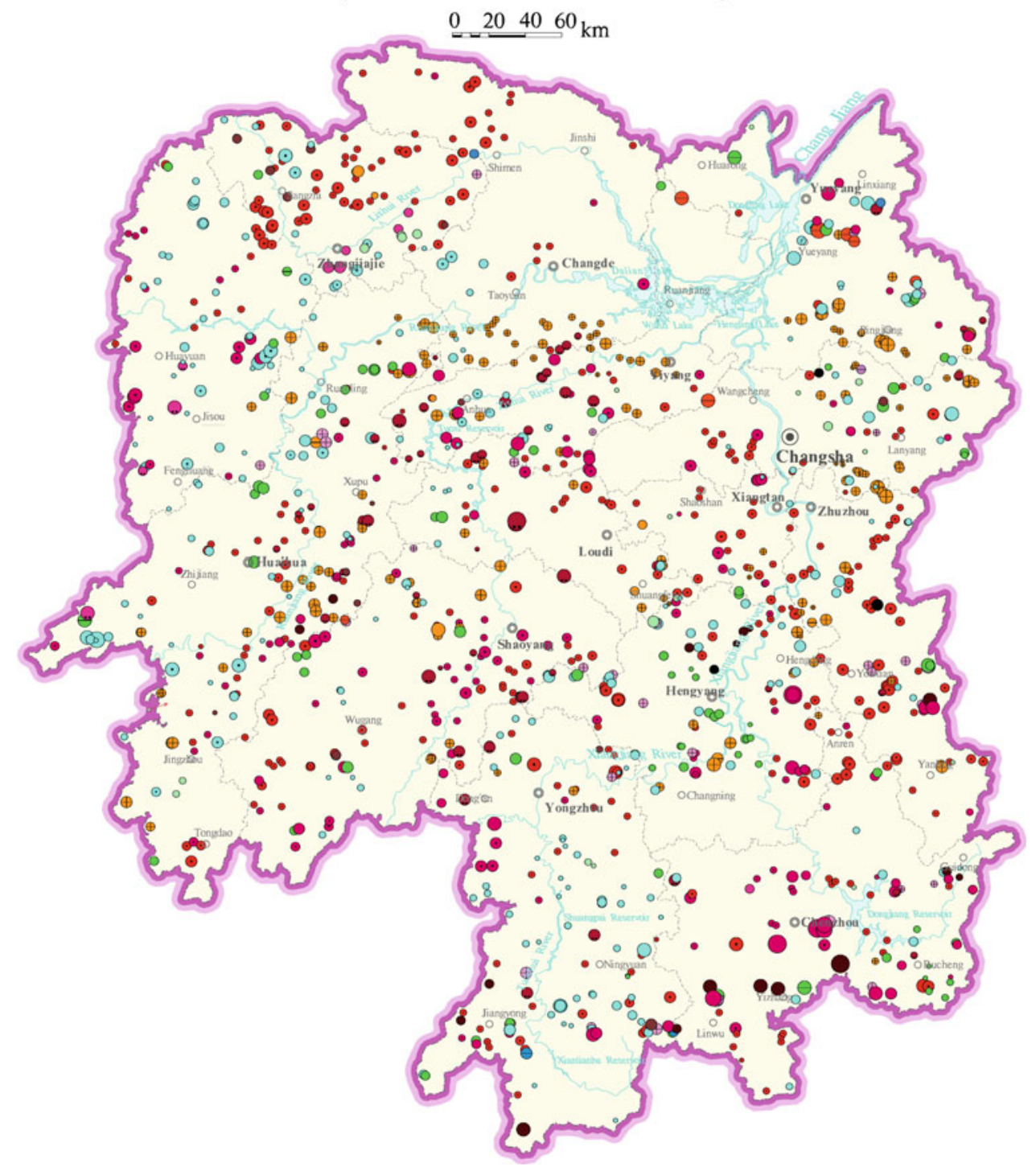


Deposit distribution map of Hunan Province ( energy minerals)

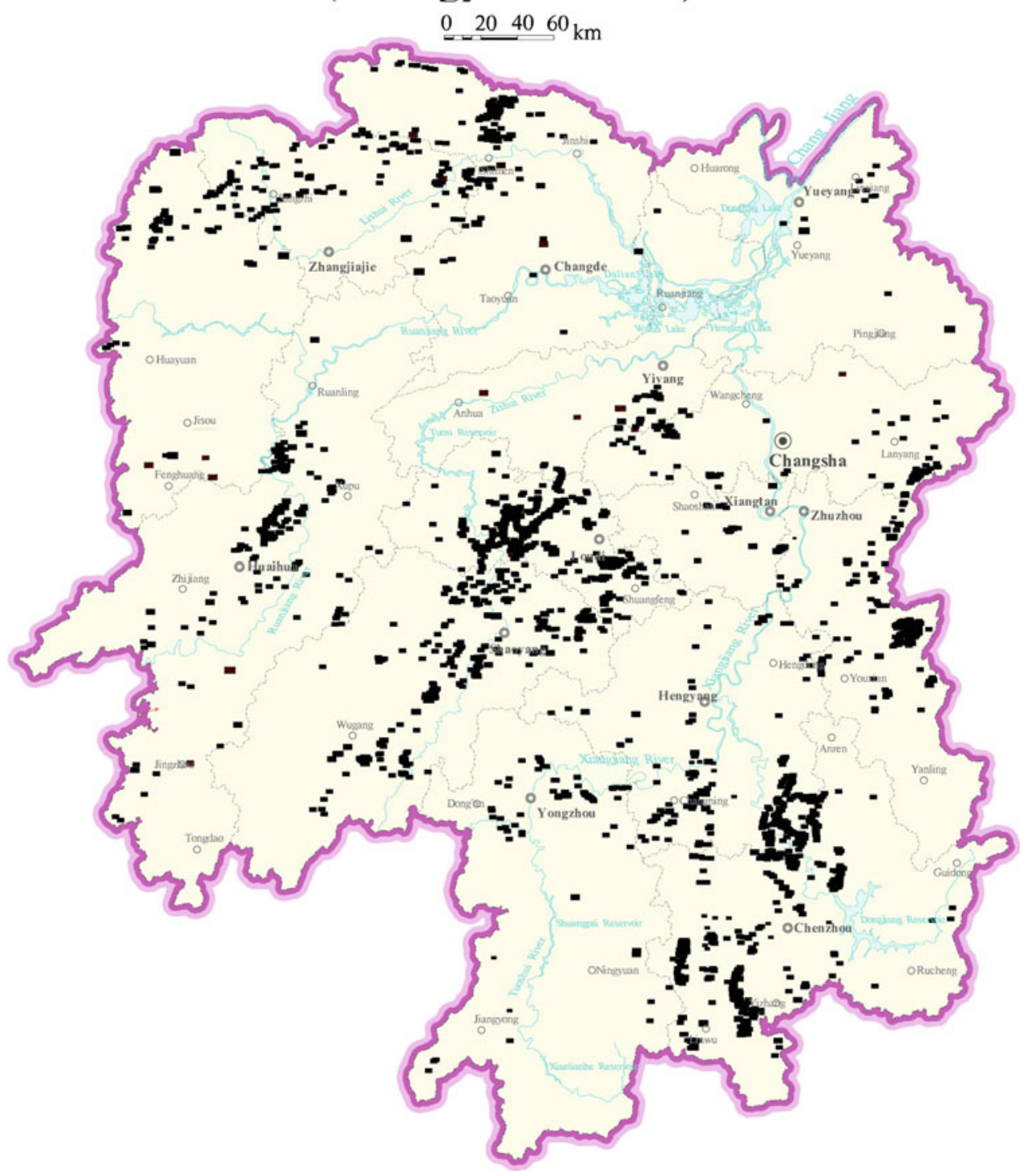


58

4 Maps of Mineral Deposits Distribution by Provinces in China

Deposit distribution map of Hunan Province (non-metallic minerals)

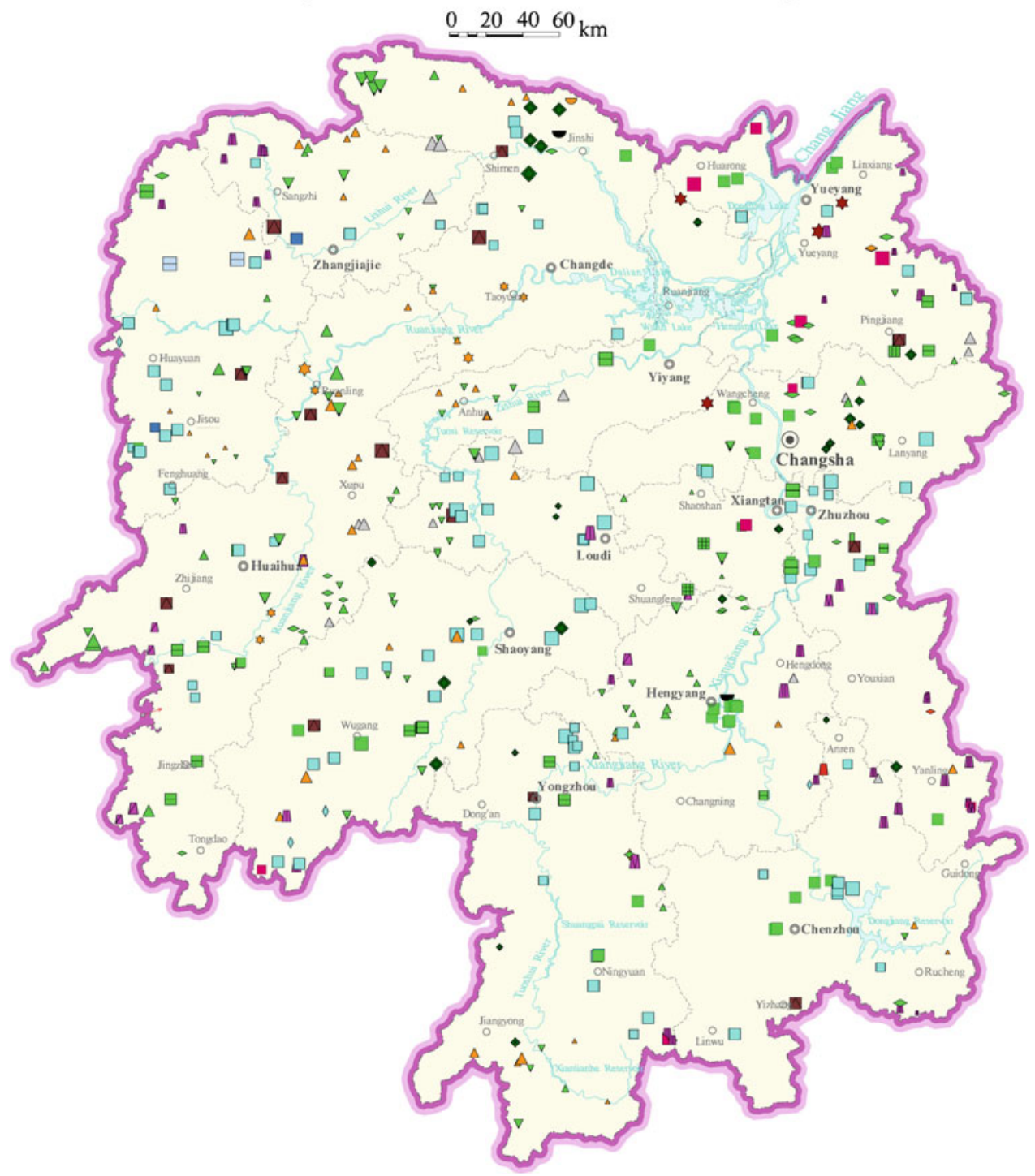


4 Maps of Mineral Deposits Distribution by Provinces in China

59

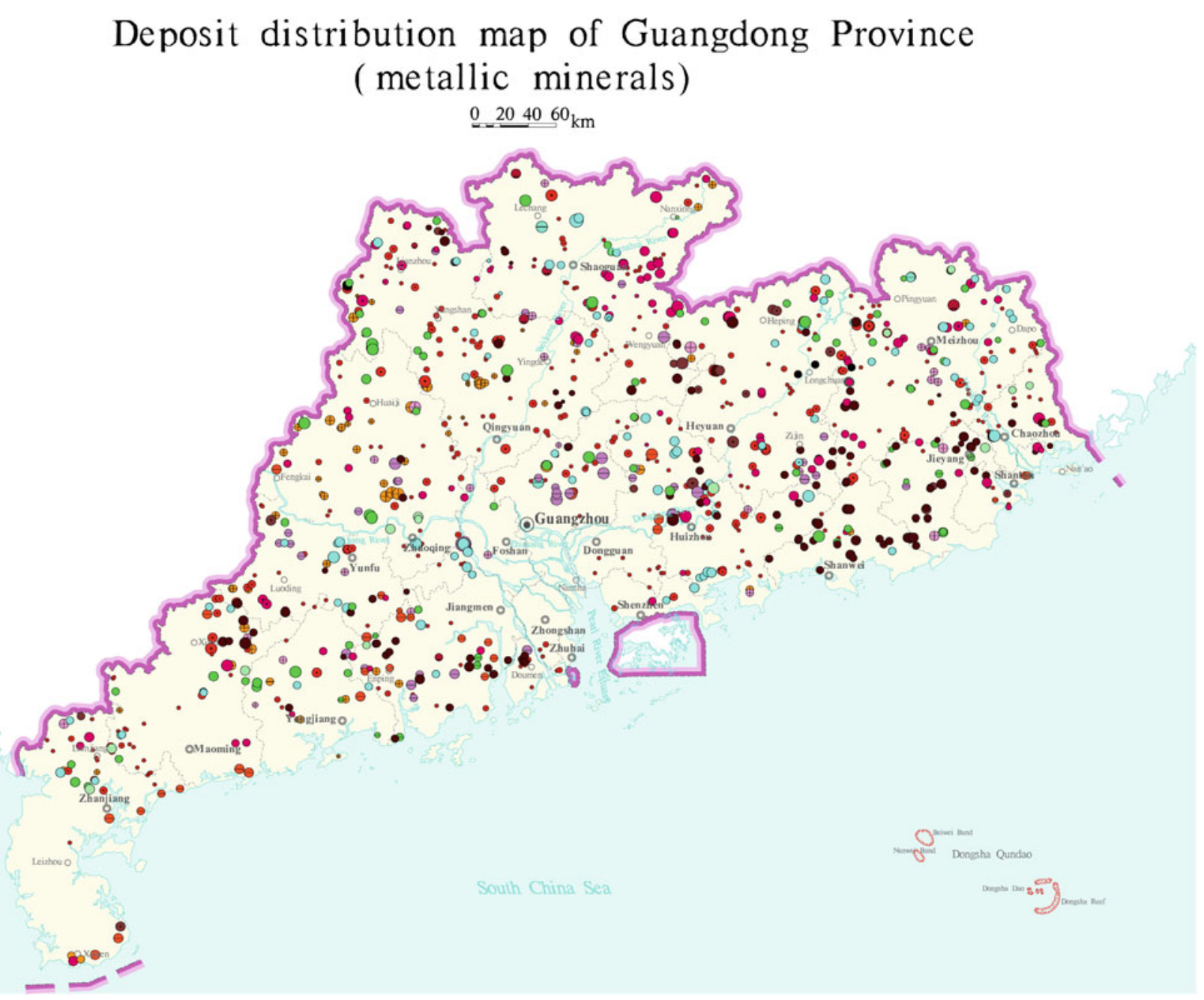




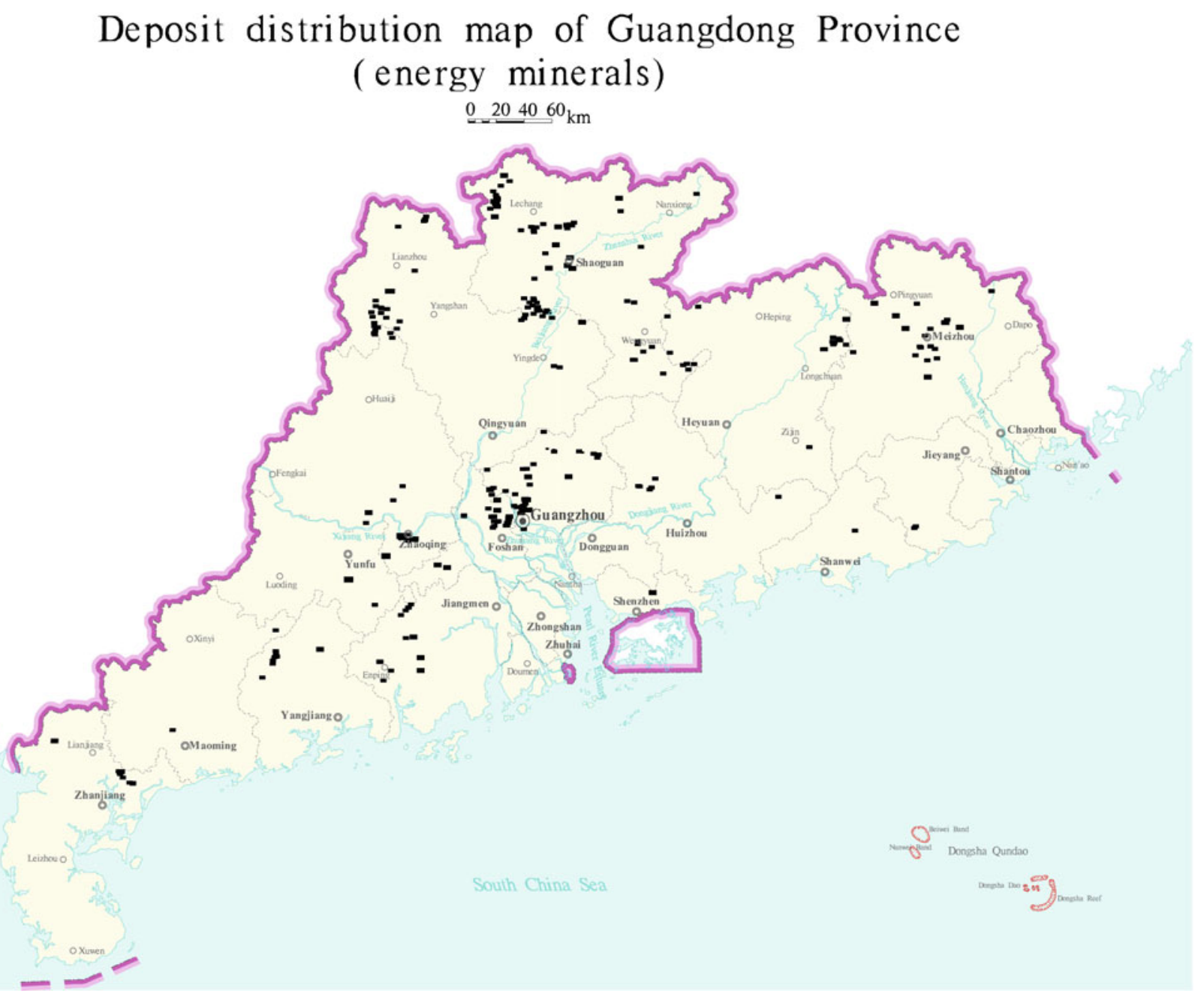




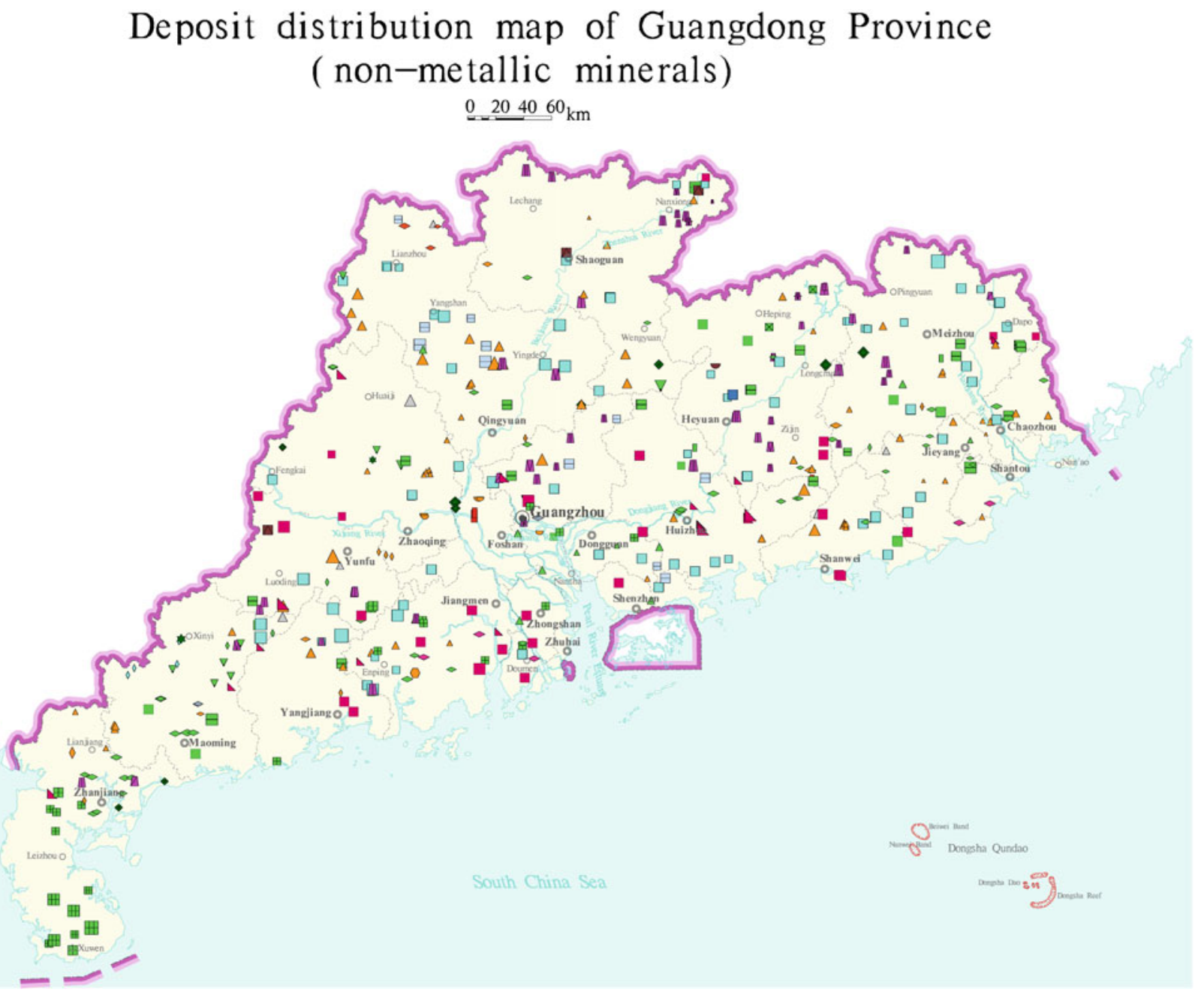


Deposit distribution map of Guangxizhuangzuzizhiqu Region ( metallic minerals) $\underline{0.20 \quad 40 \quad 60} \mathrm{~km}$

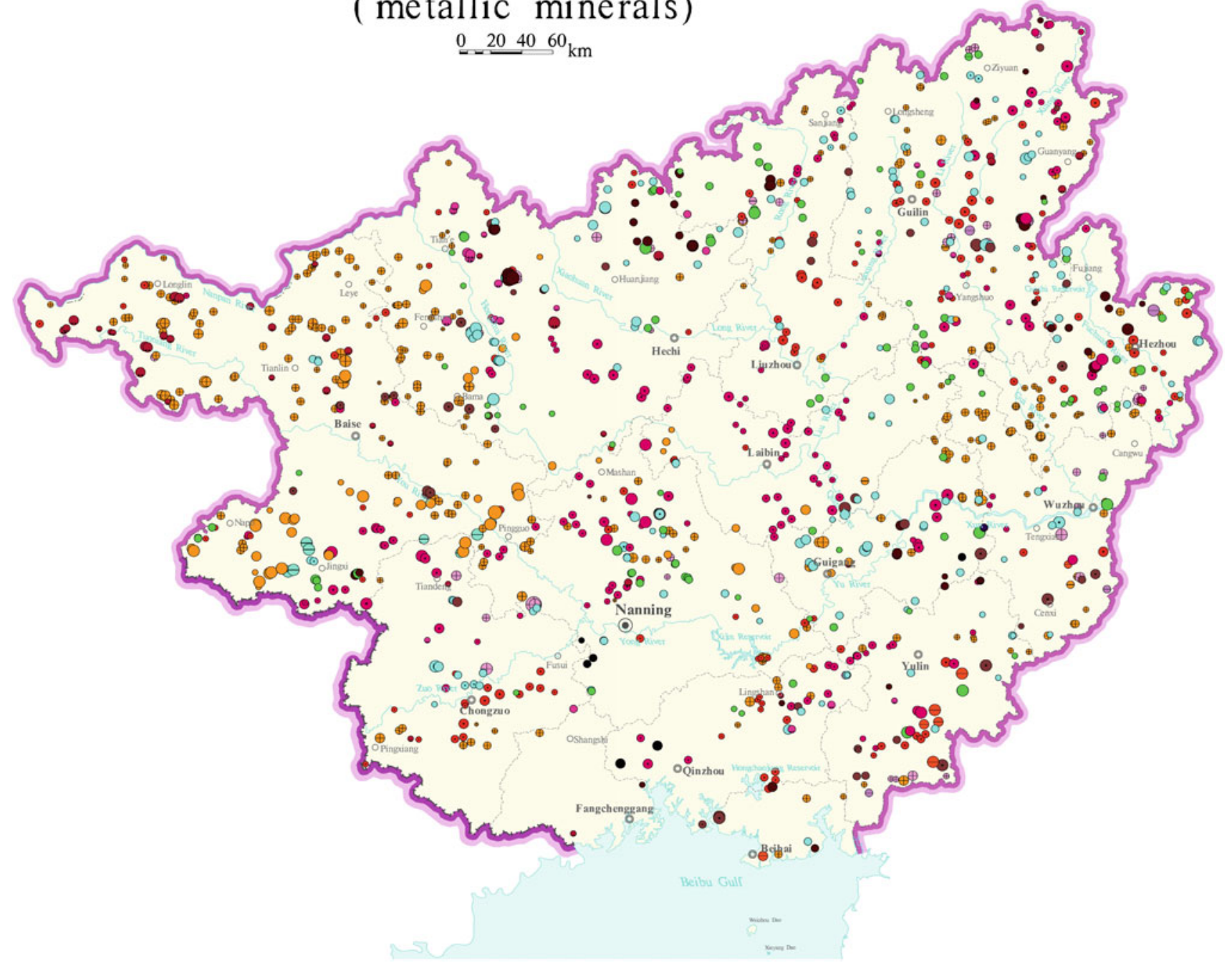


Deposit distribution map of Guangxizhuangzuzizhiqu Region (energy minerals)

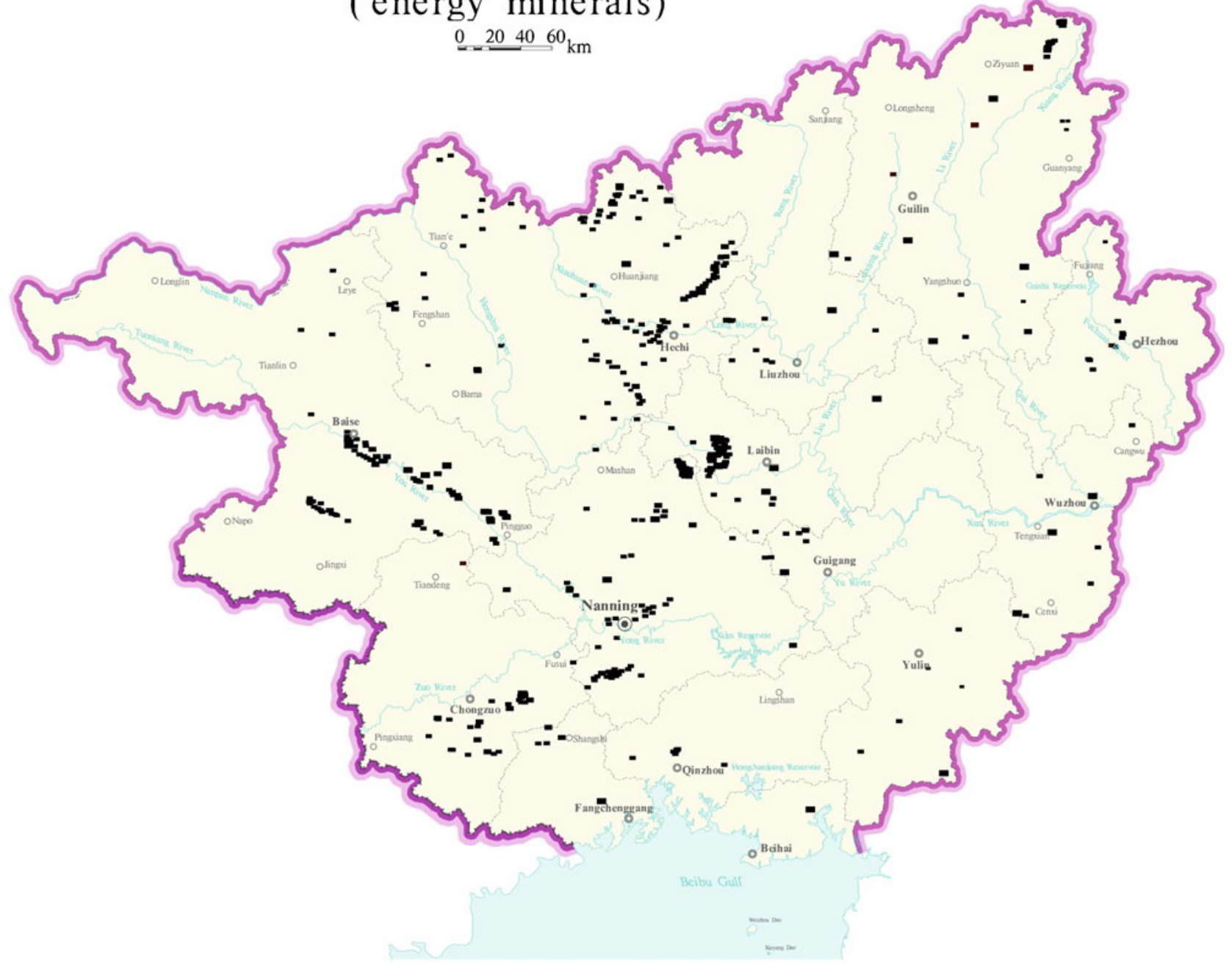


Deposit distribution map of Guangxizhuangzuzizhiqu Region ( non-metallic minerals)

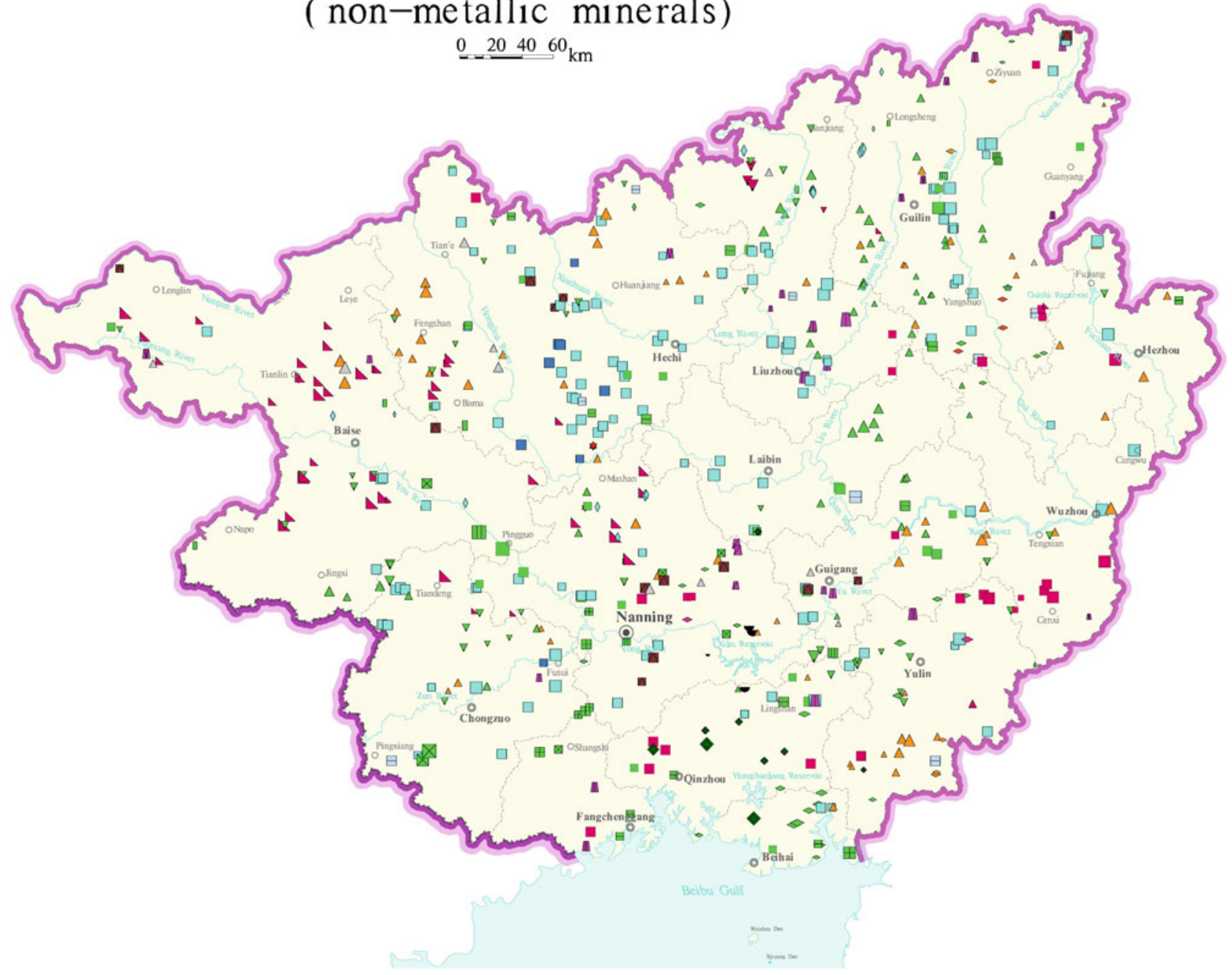


Deposit distribution map of Hainan Province $0 \quad 10 \quad 20 \quad 30$
-

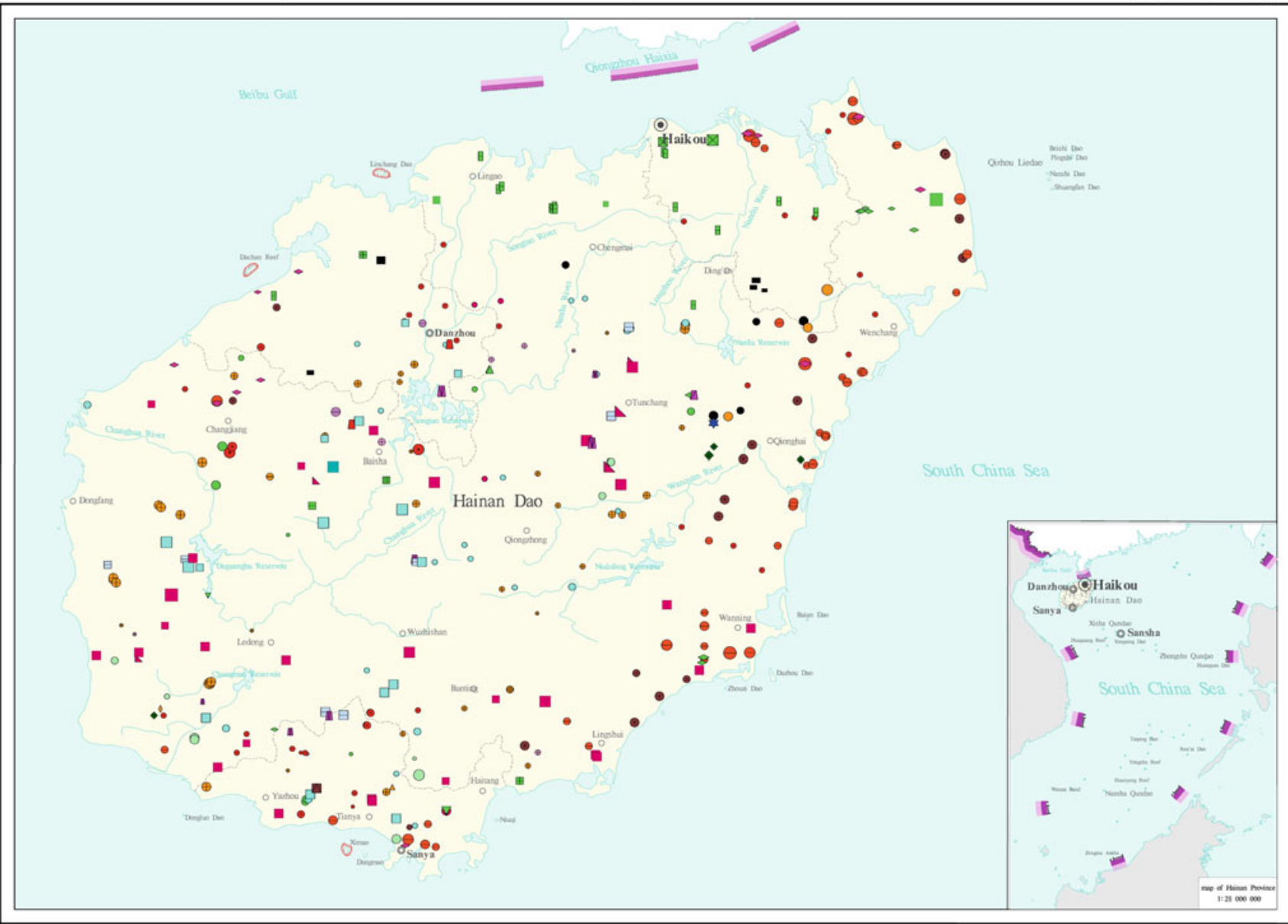




\section{Deposit distribution map of Chongqing Shi, China} (metallic minerals)

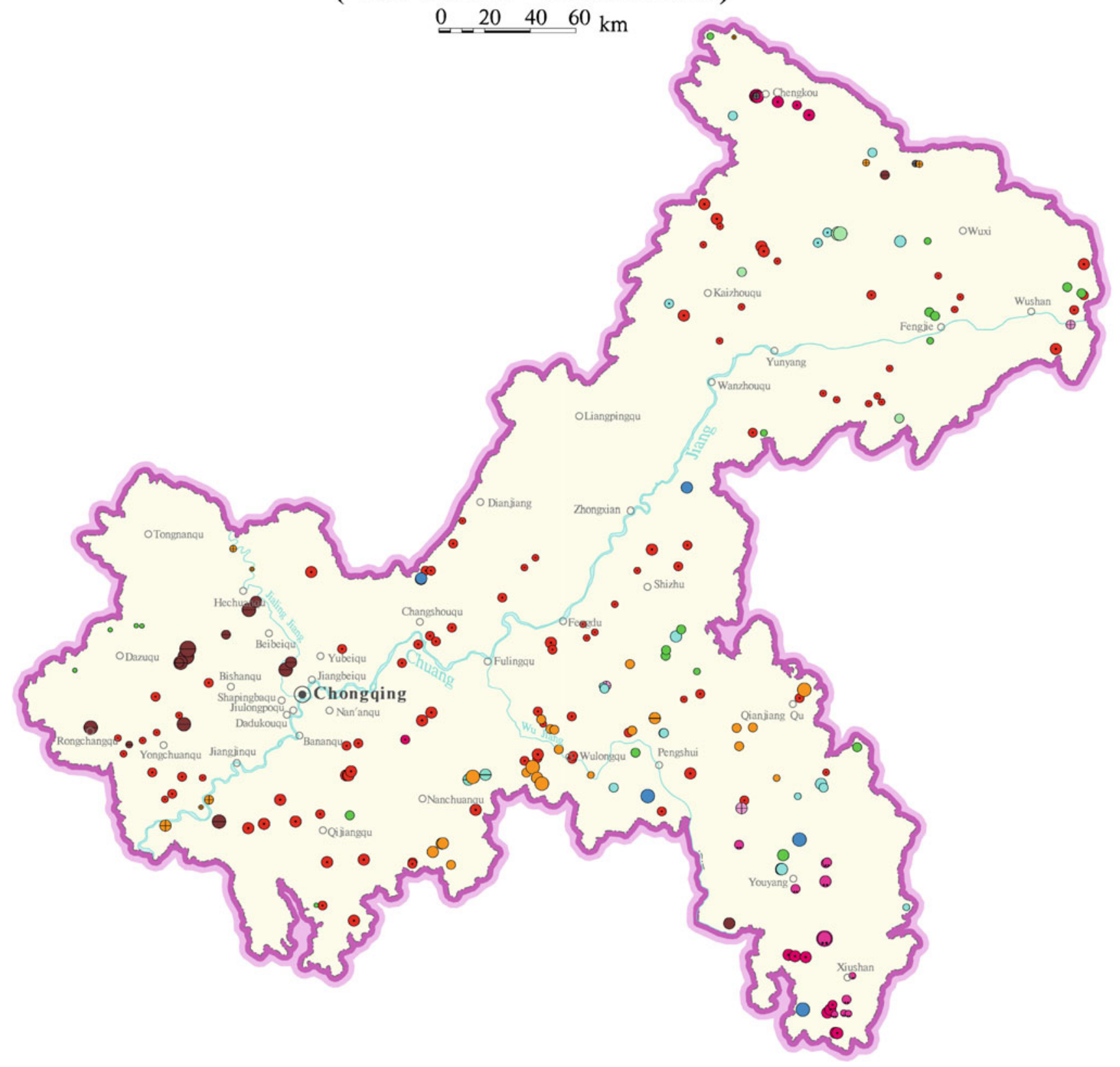


Deposit distribution map of Chongqing Shi, China ( energy minerals)

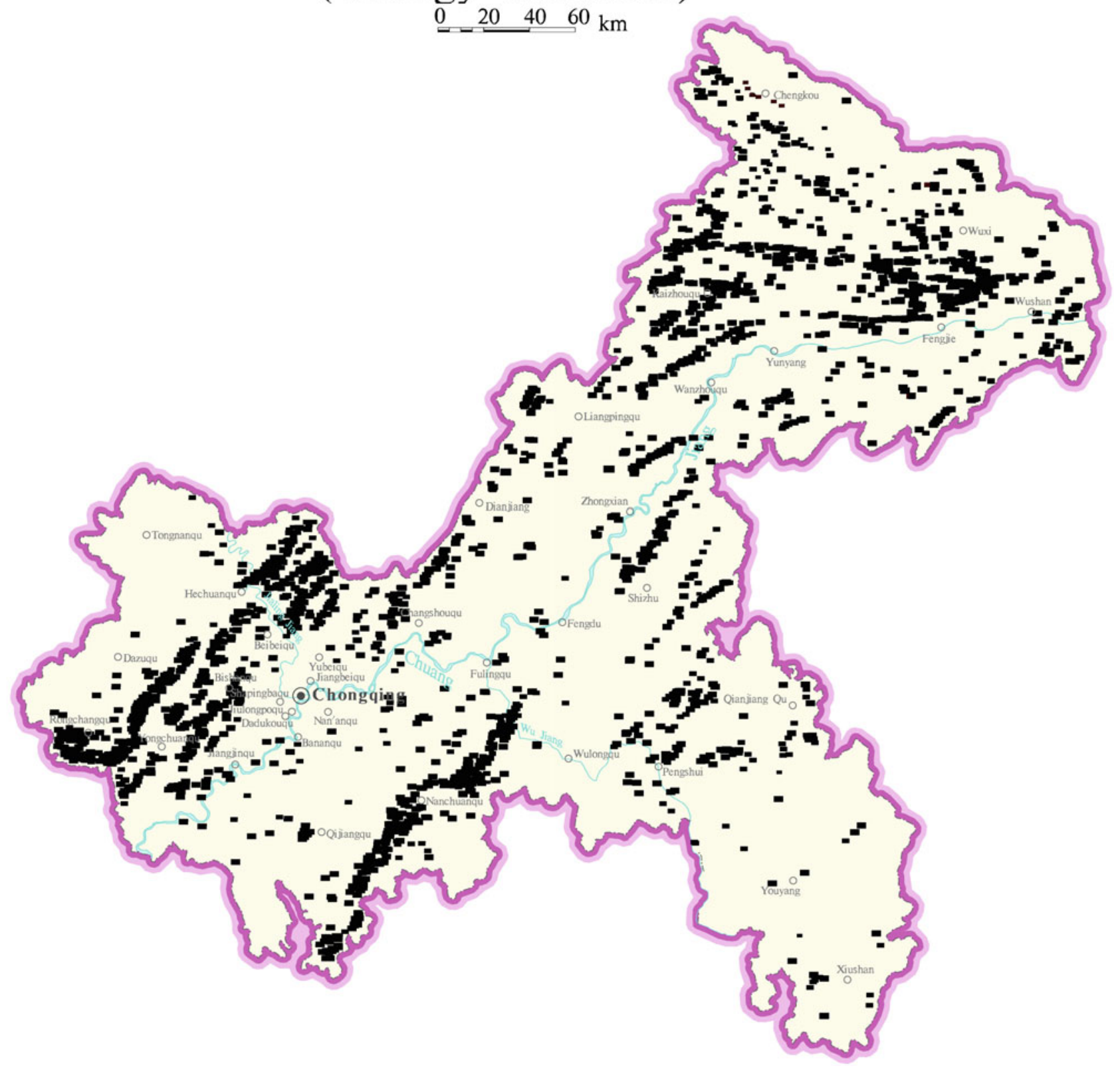


Deposit distribution map of Chongqing Shi, China ( non-metallic minerals)

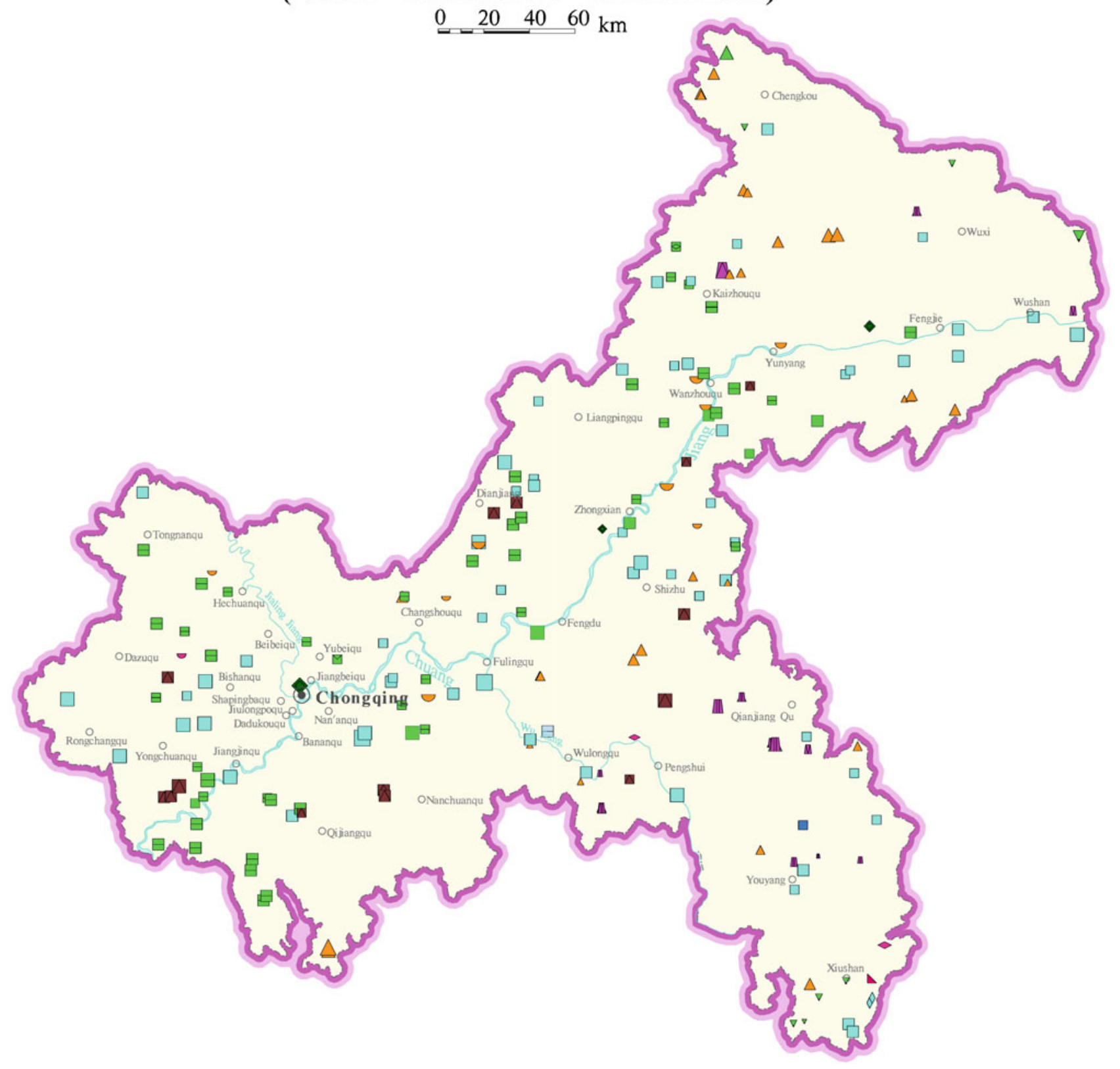


Deposit distribution map of Sichuan Province

(metallic minerals)

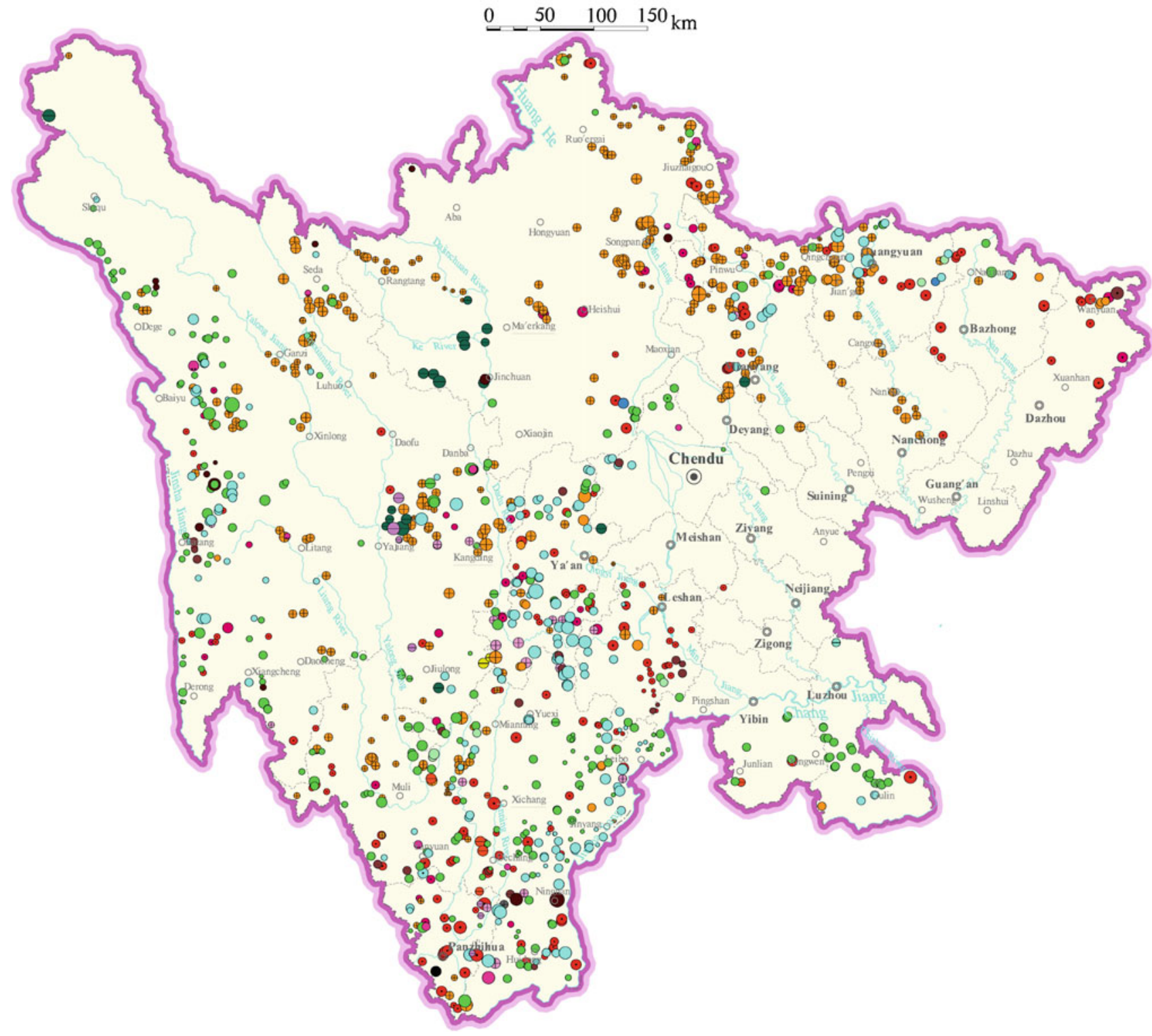


Deposit distribution map of Sichuan Province ( energy minerals)

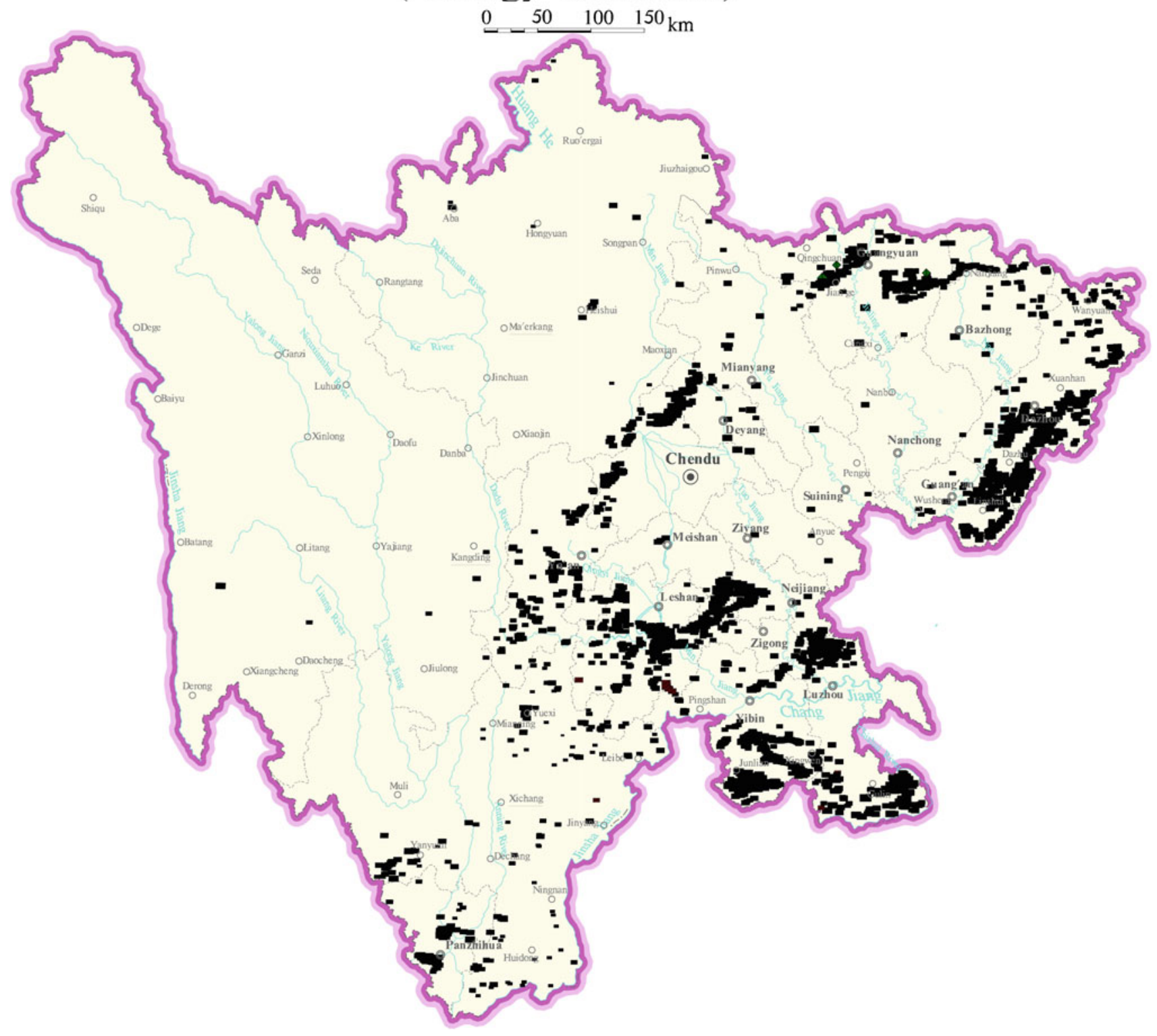


Deposit distribution map of Sichuan Province (non-metallic minerals)

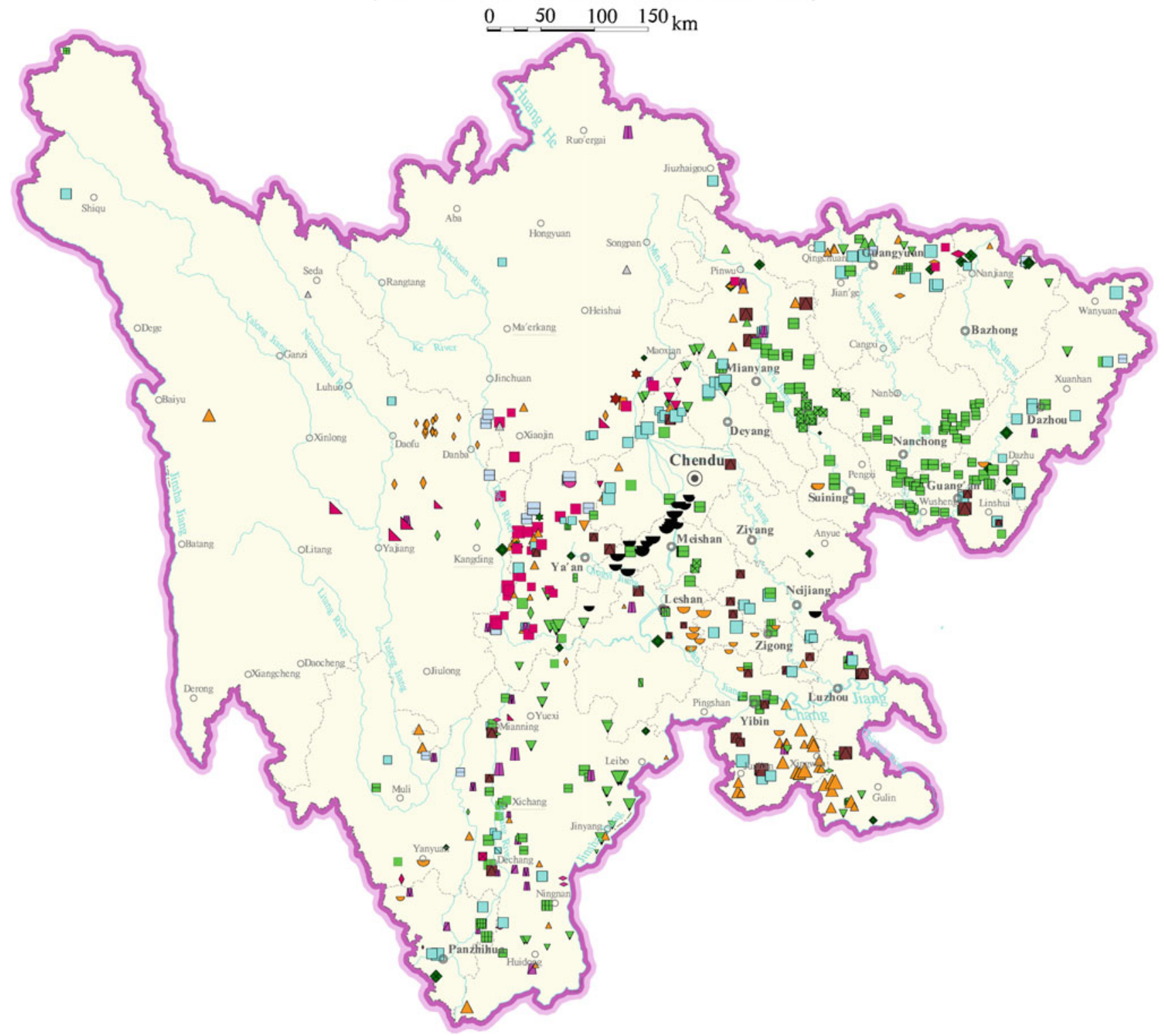


Deposit distribution map of Guizhou Province (metallic minerals)

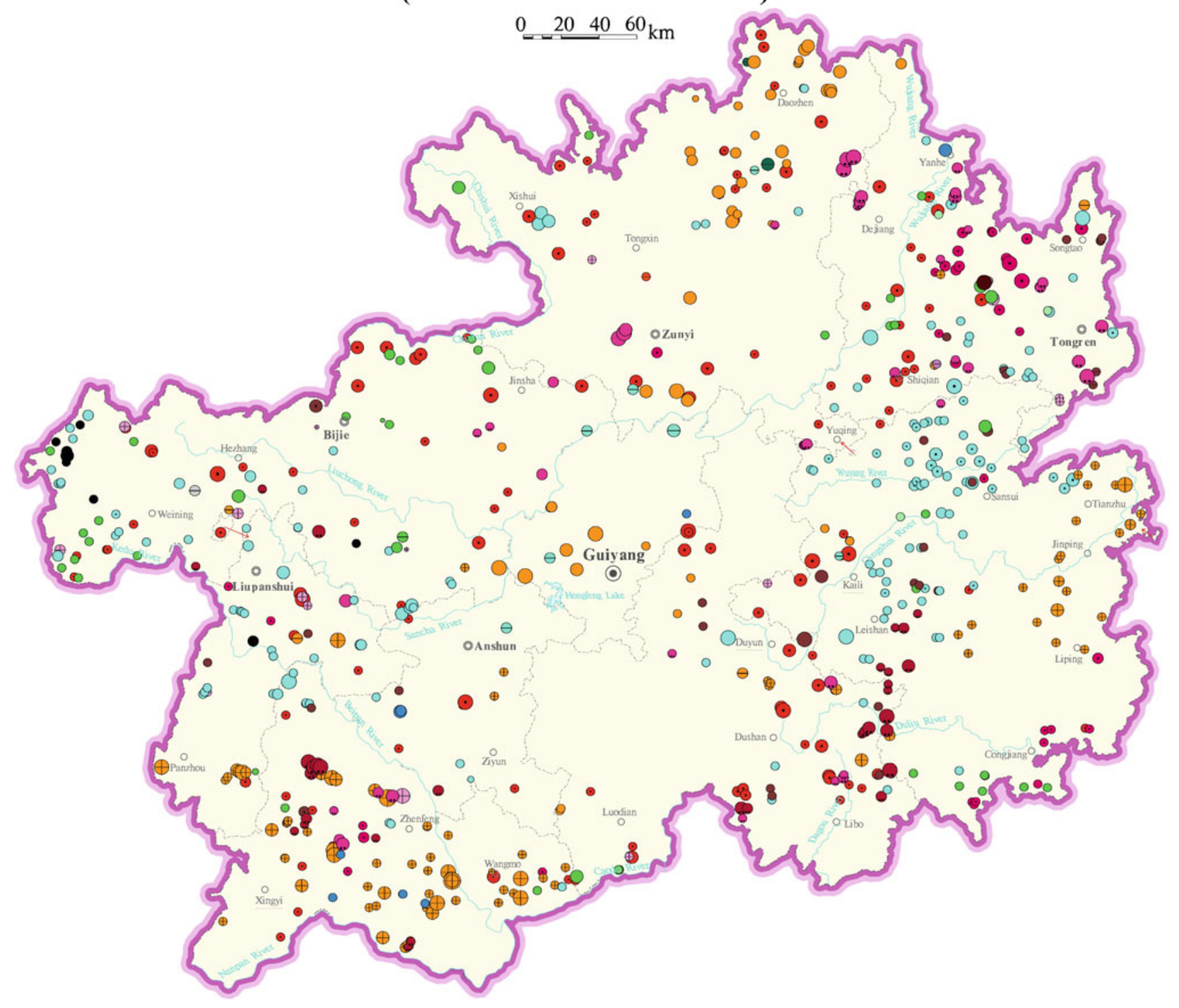


Deposit distribution map of Guizhou Province ( energy minerals)

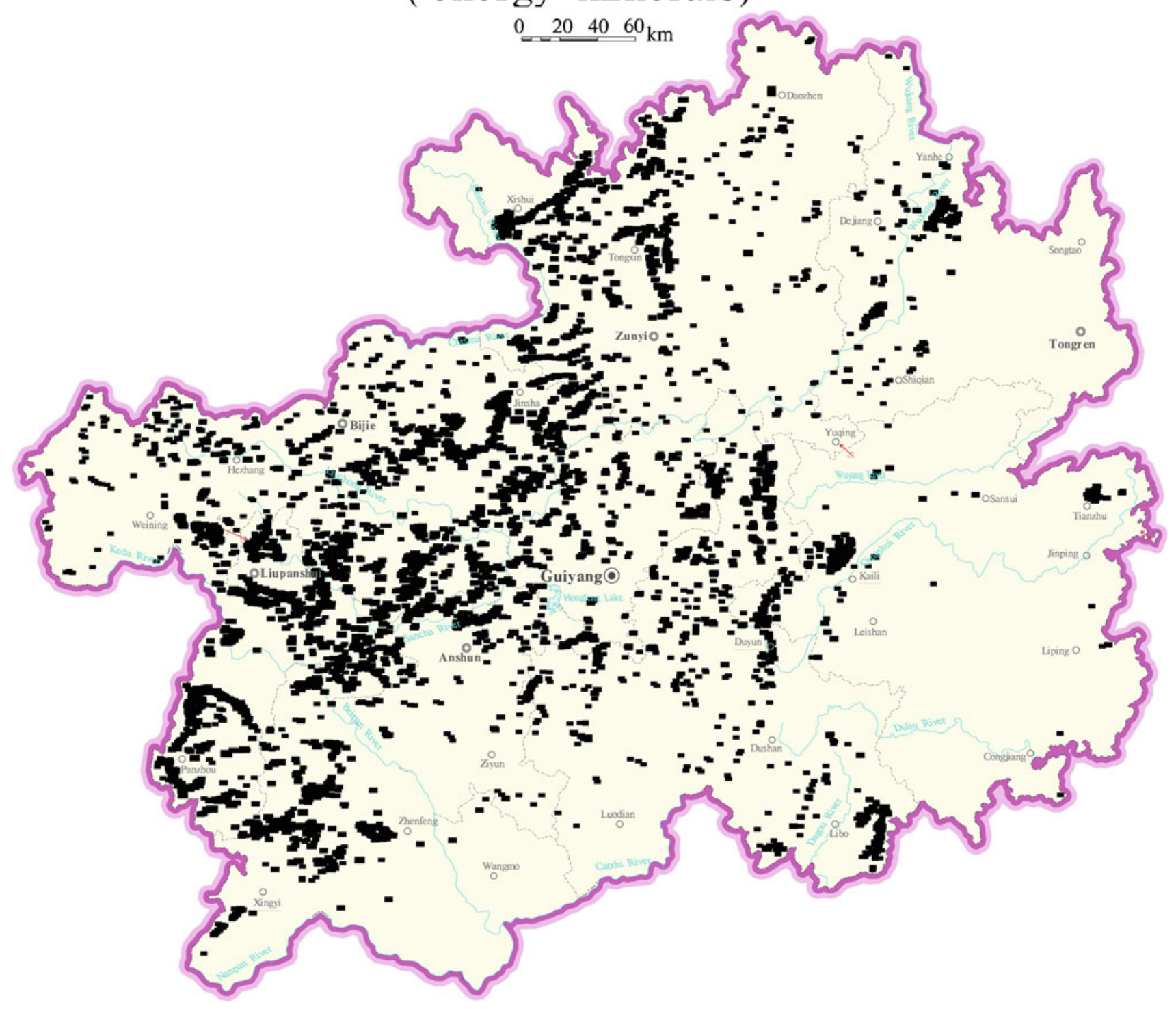




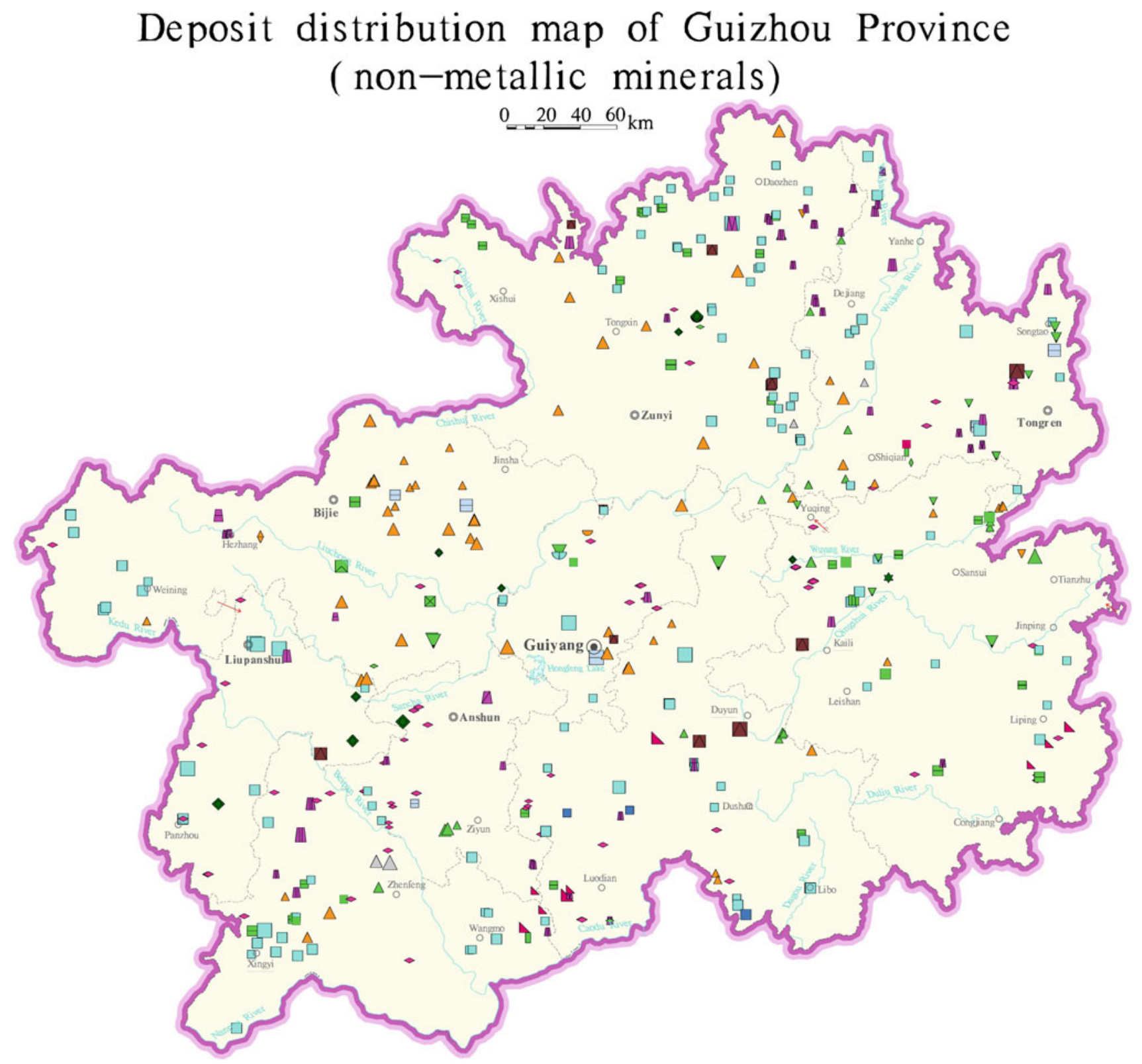




\section{Deposit distribution map of Yunnan Province}

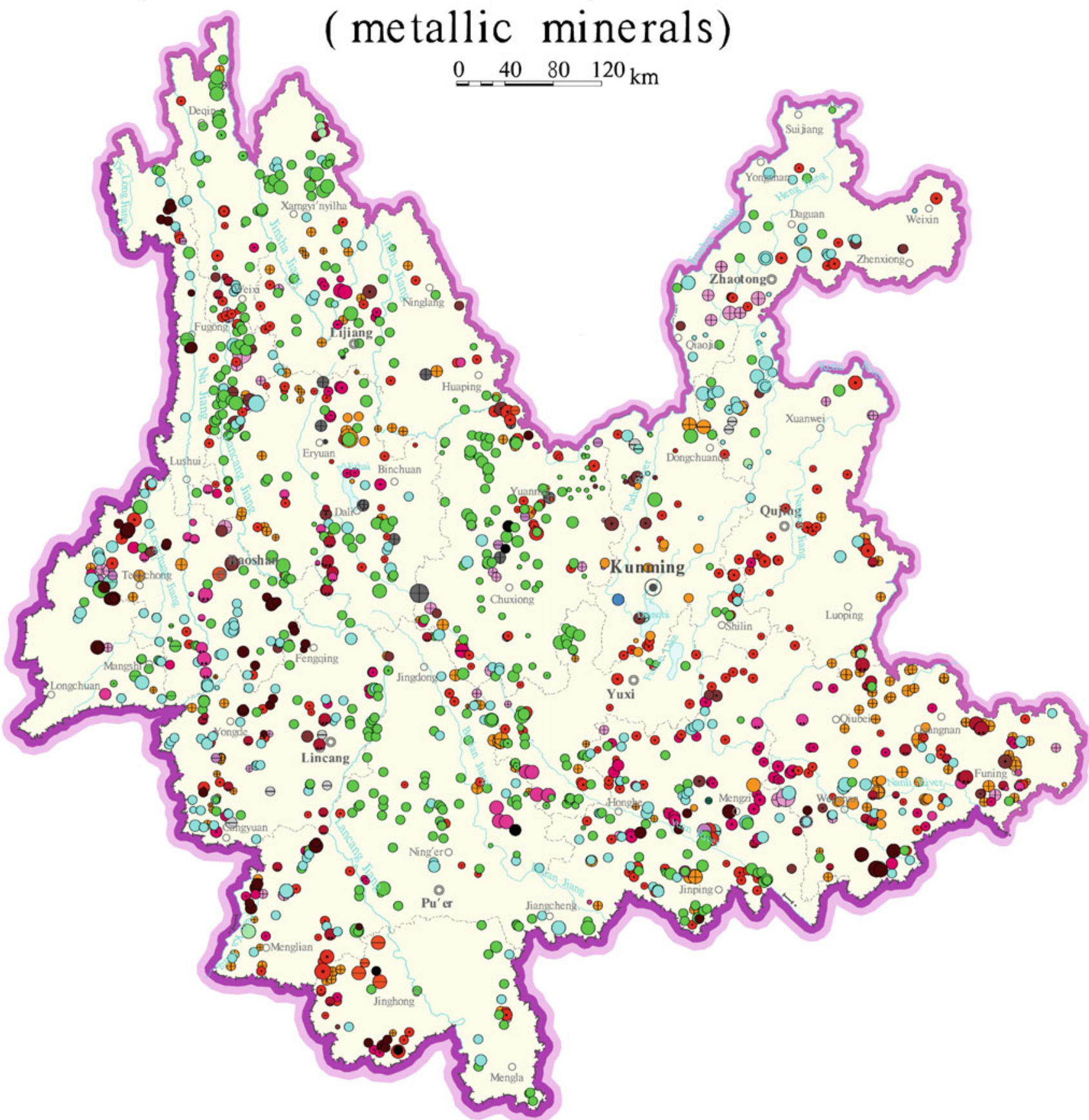




\section{Deposit distribution map of Yunnan Province}

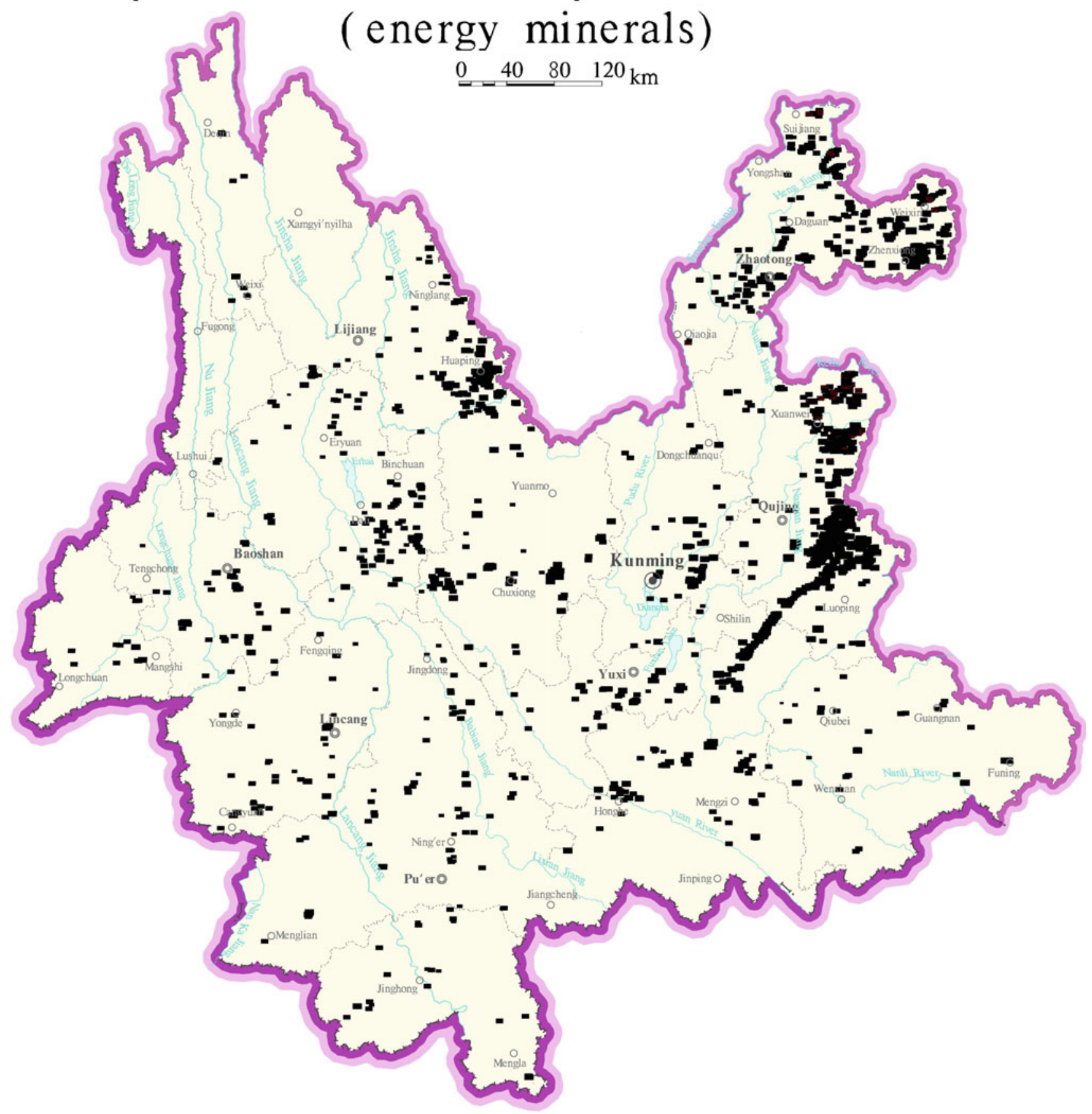


Deposit distribution map of Yunnan Province

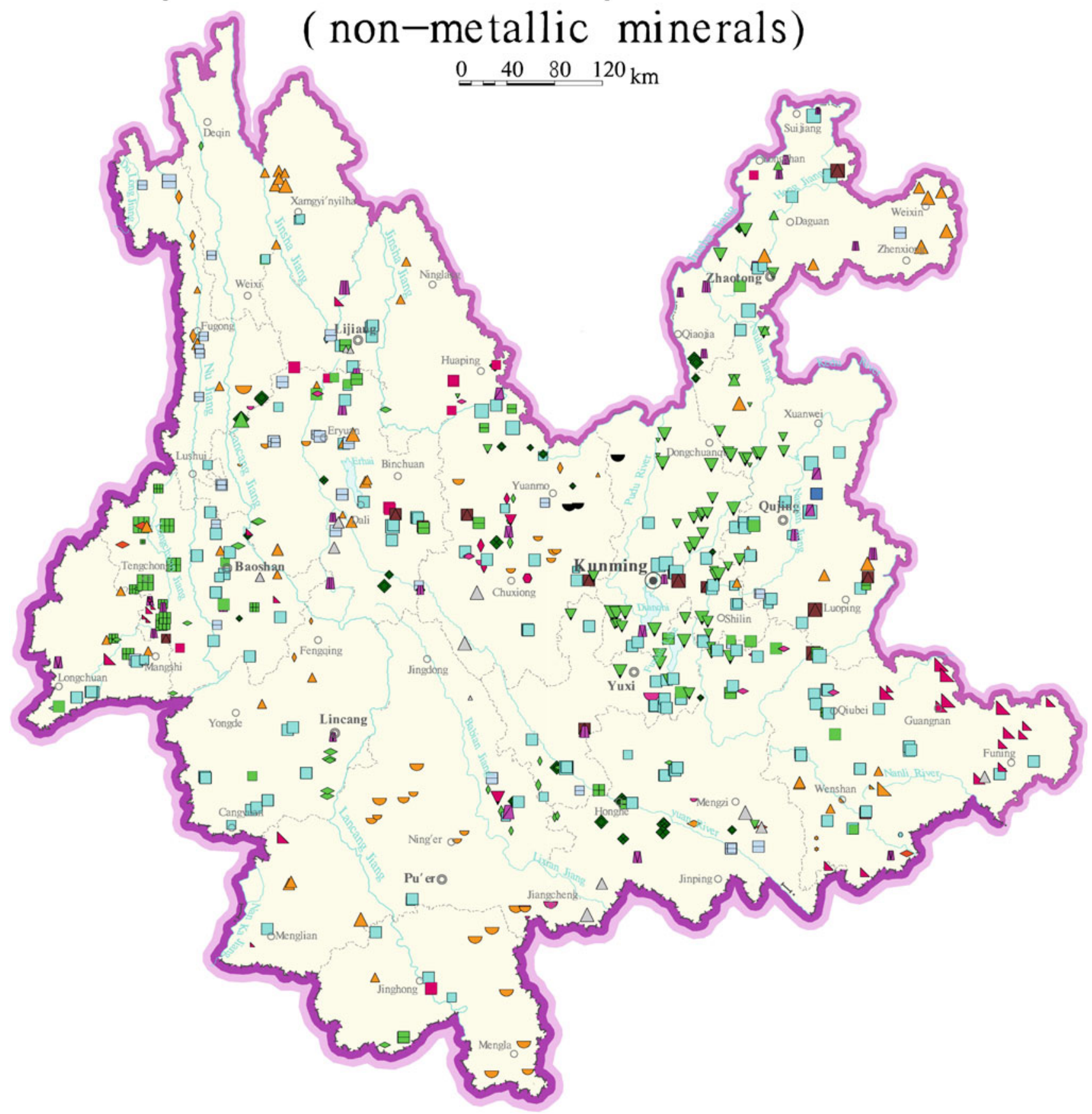


Deposit distribution map of Xizangzizhiqu Region (metallic minerals)

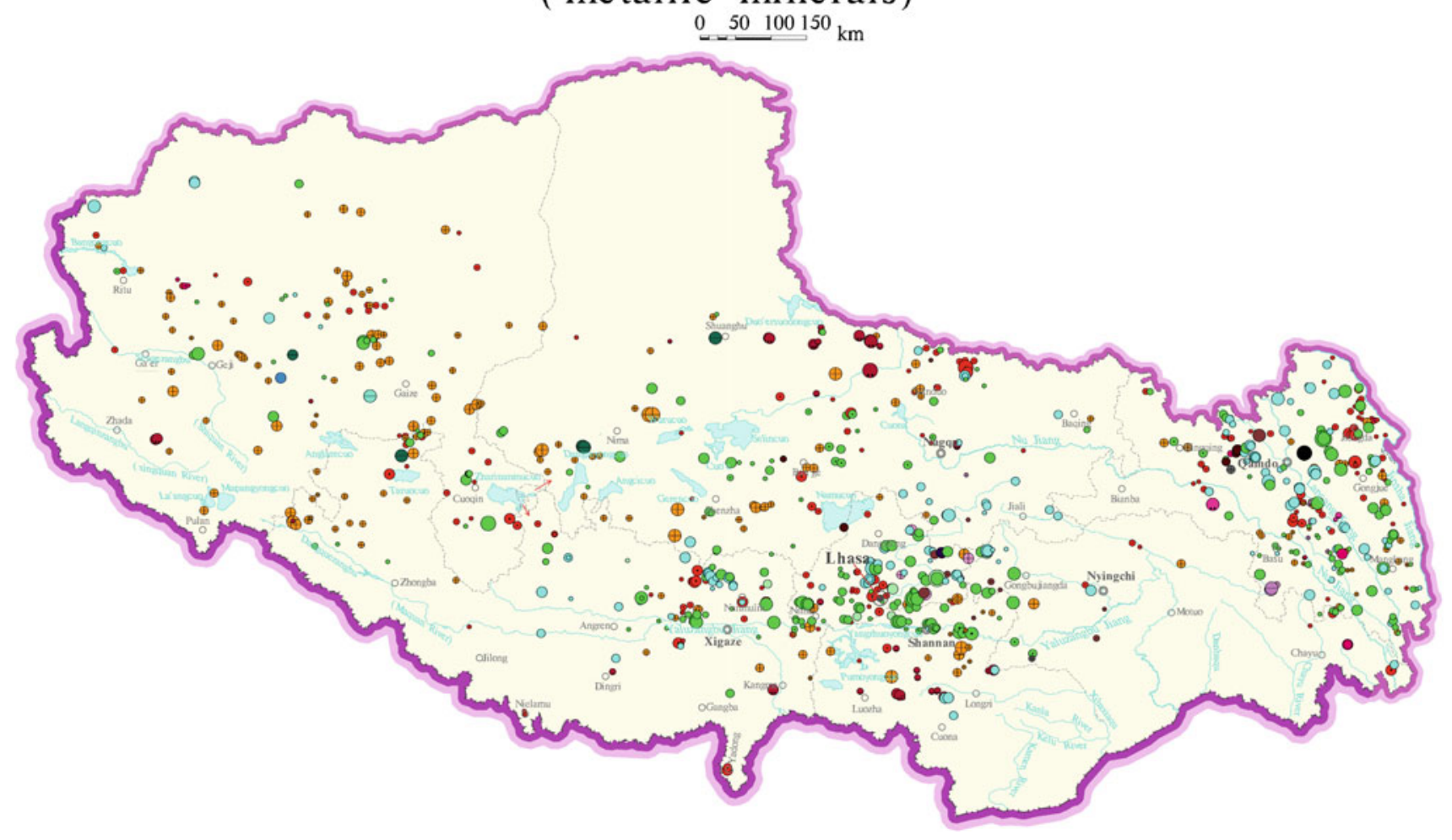

Deposit distribution map of Xizangzizhiqu Region (non-metallic minerals and energy minerals)

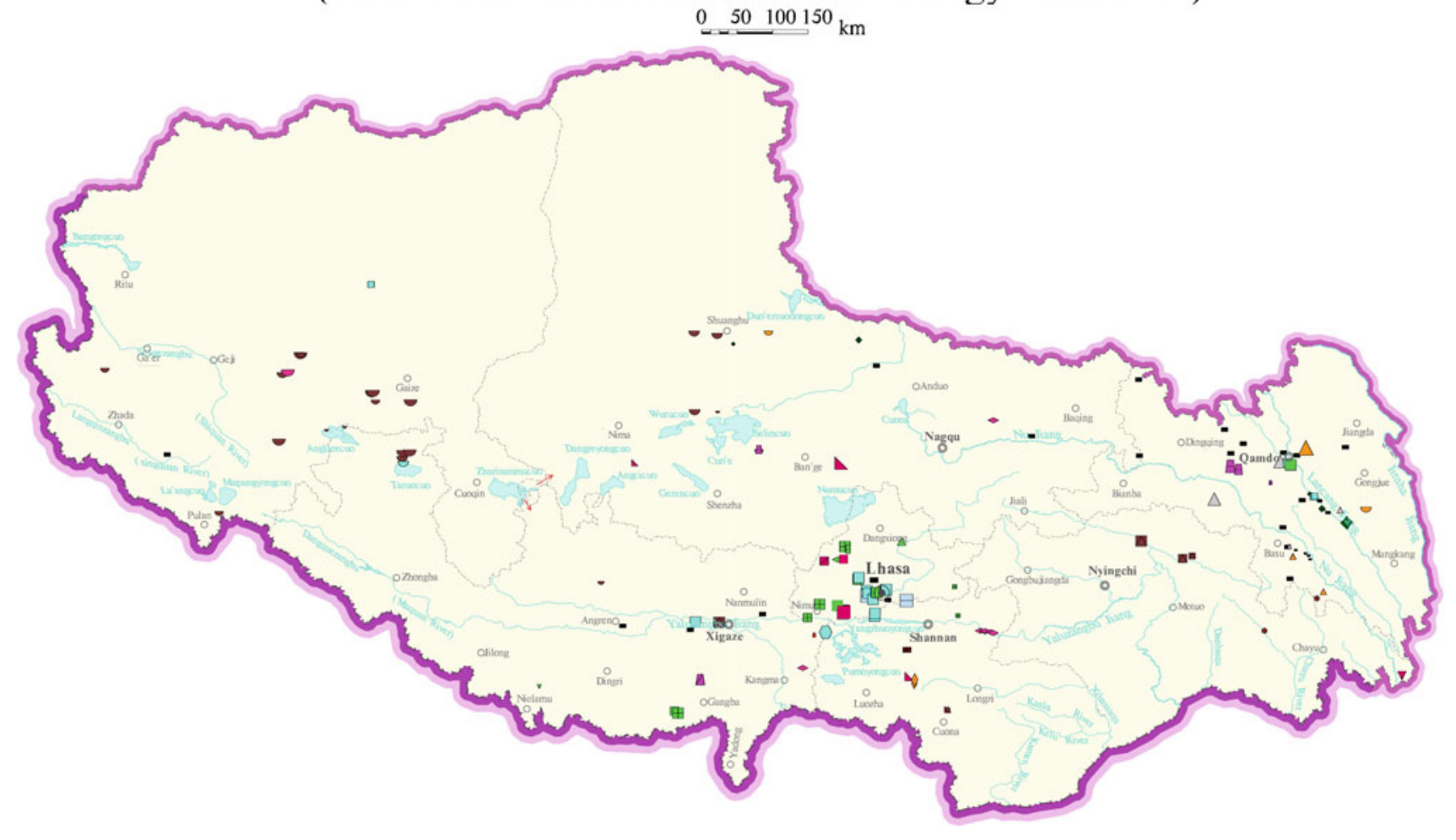




\section{Deposit distribution map of Shaanxi Province} (metallic minerals)

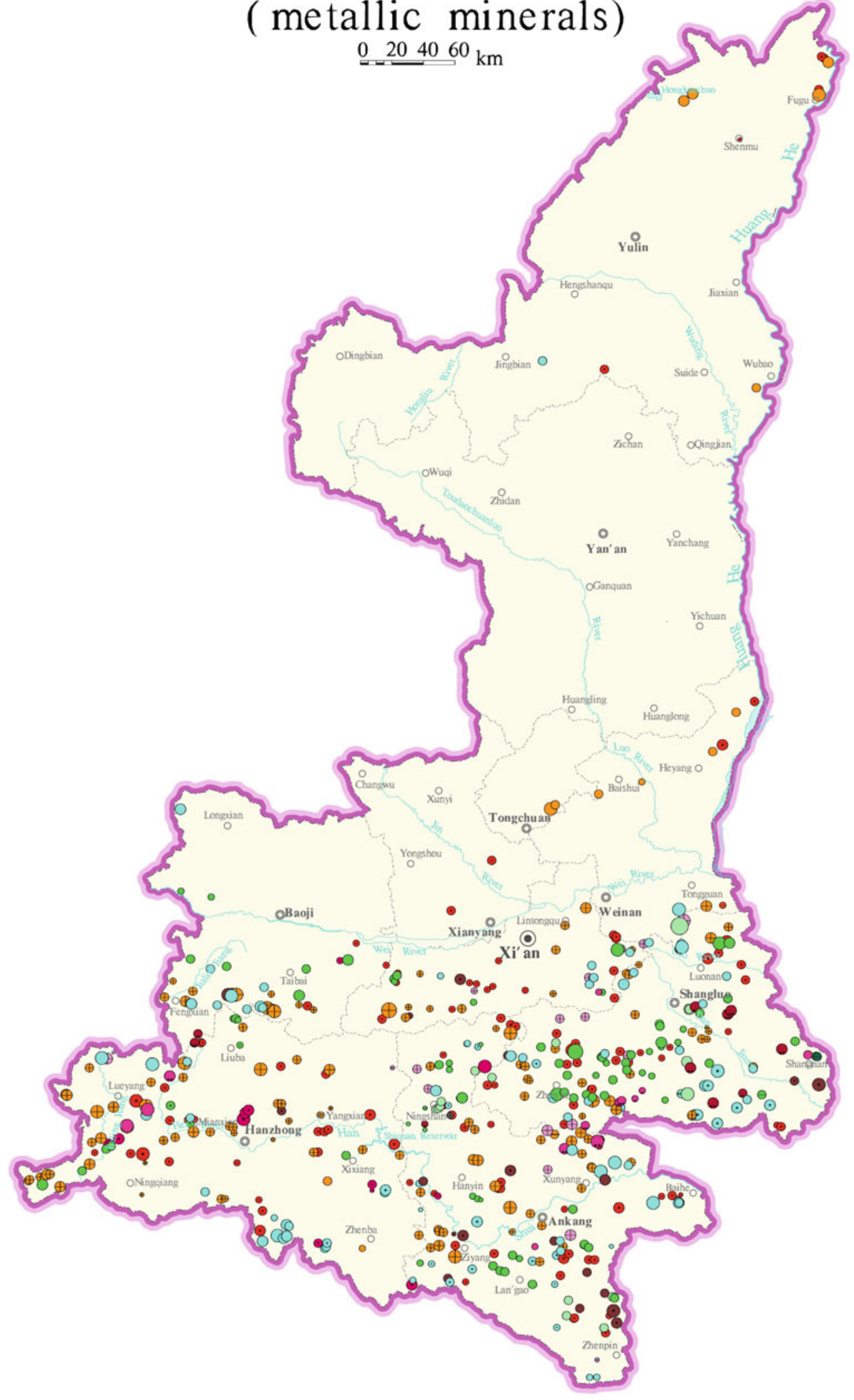


Deposit distribution map of Shaanxi Province

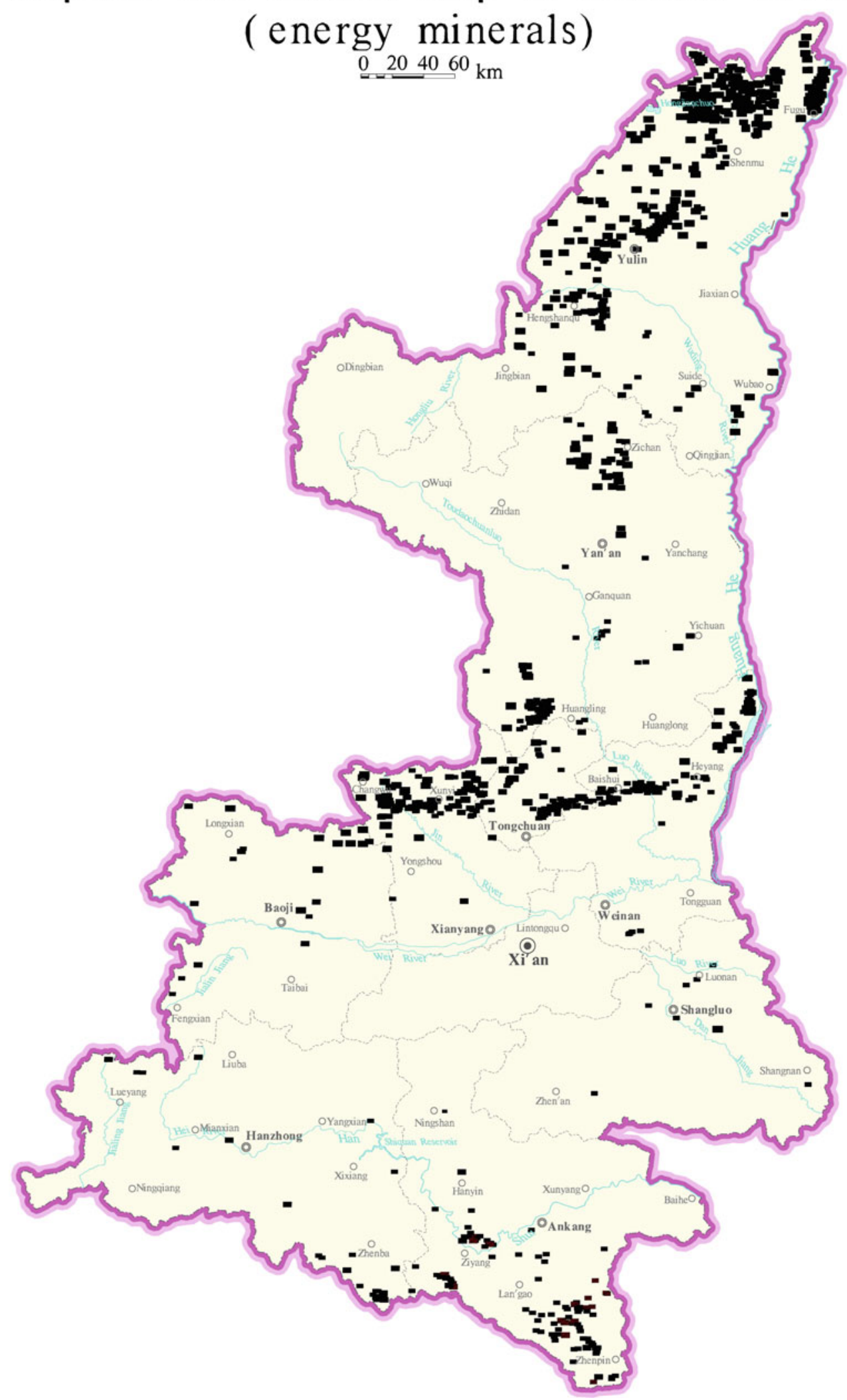




\section{Deposit distribution map of Shaanxi Province}

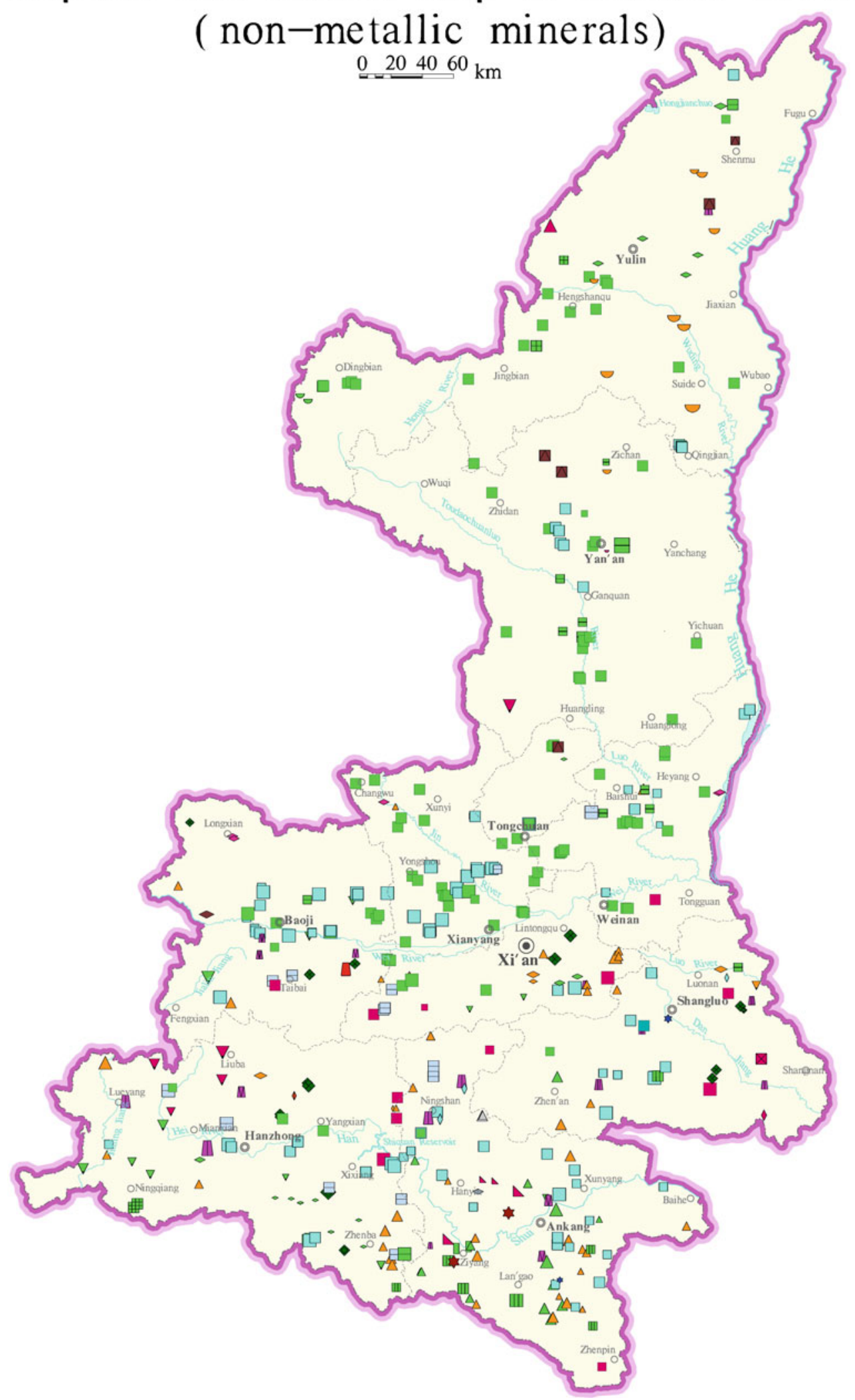




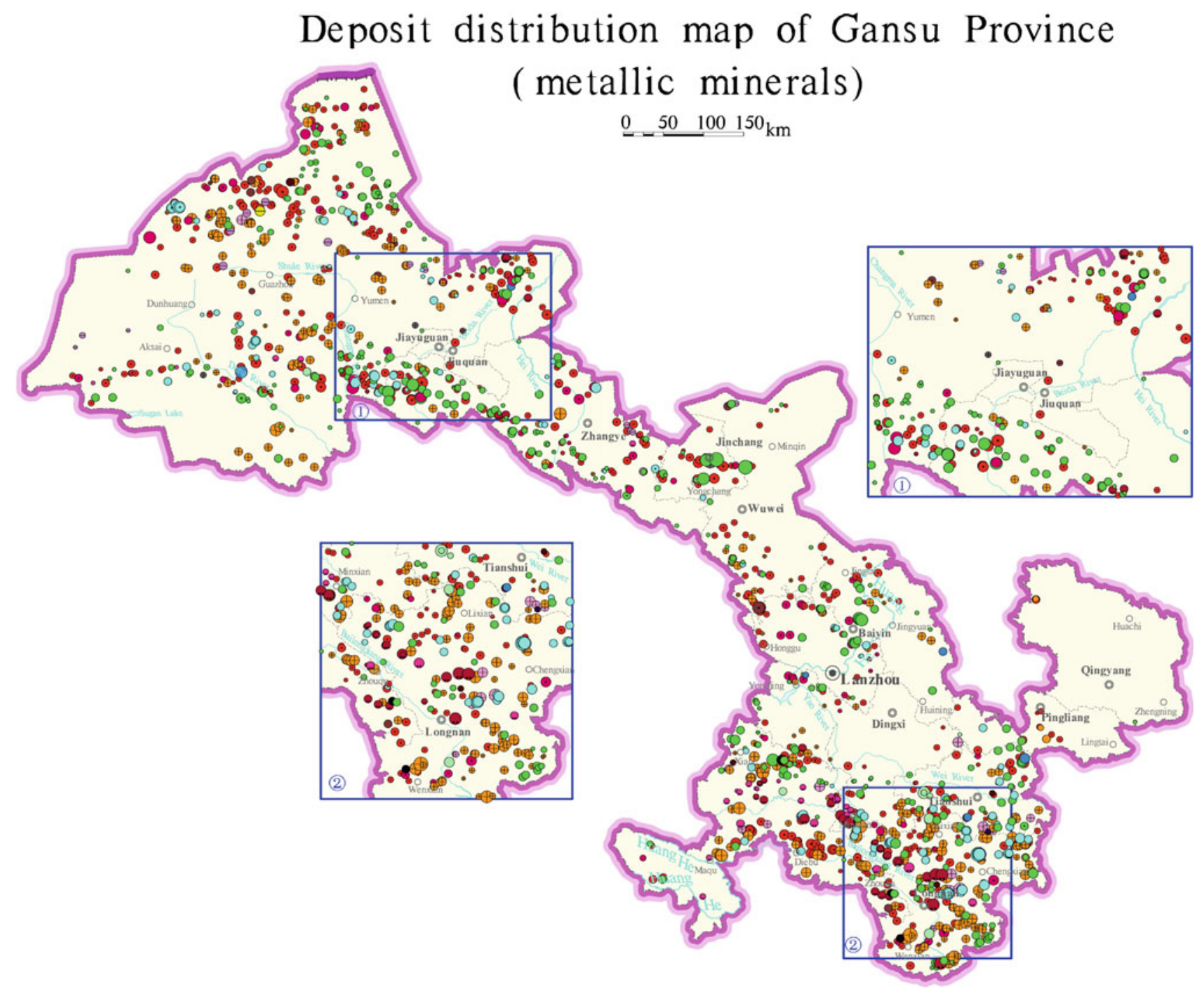




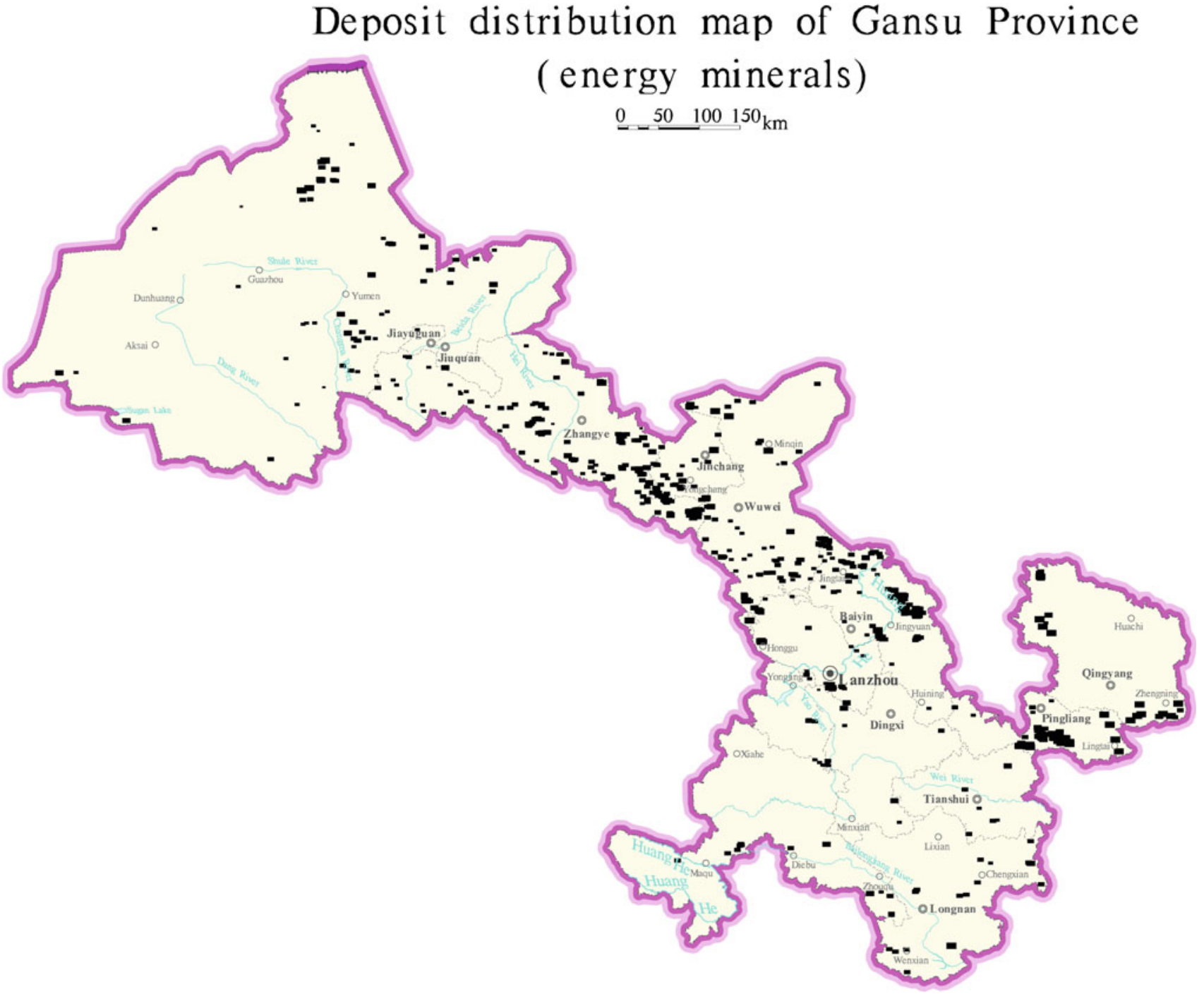




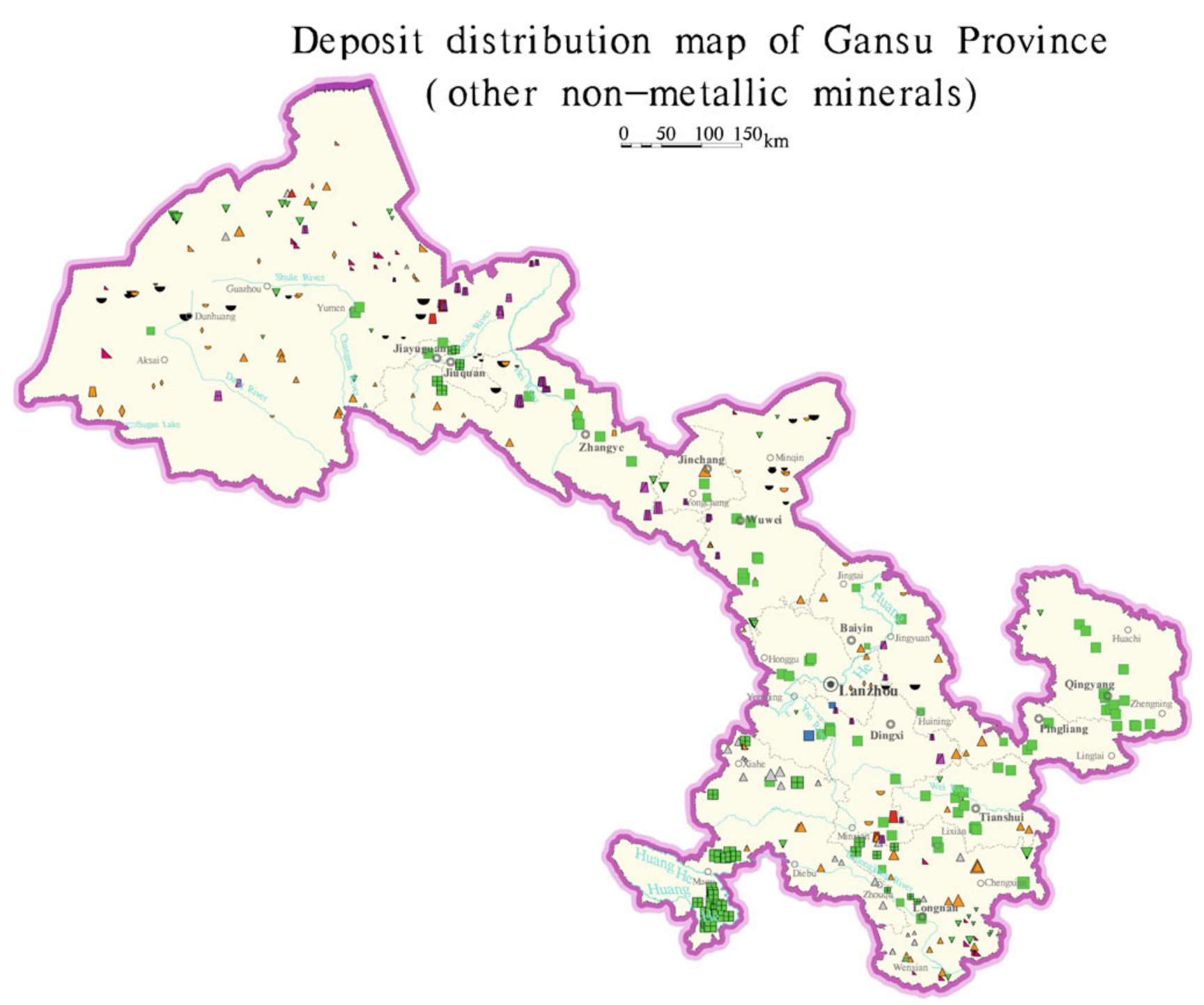




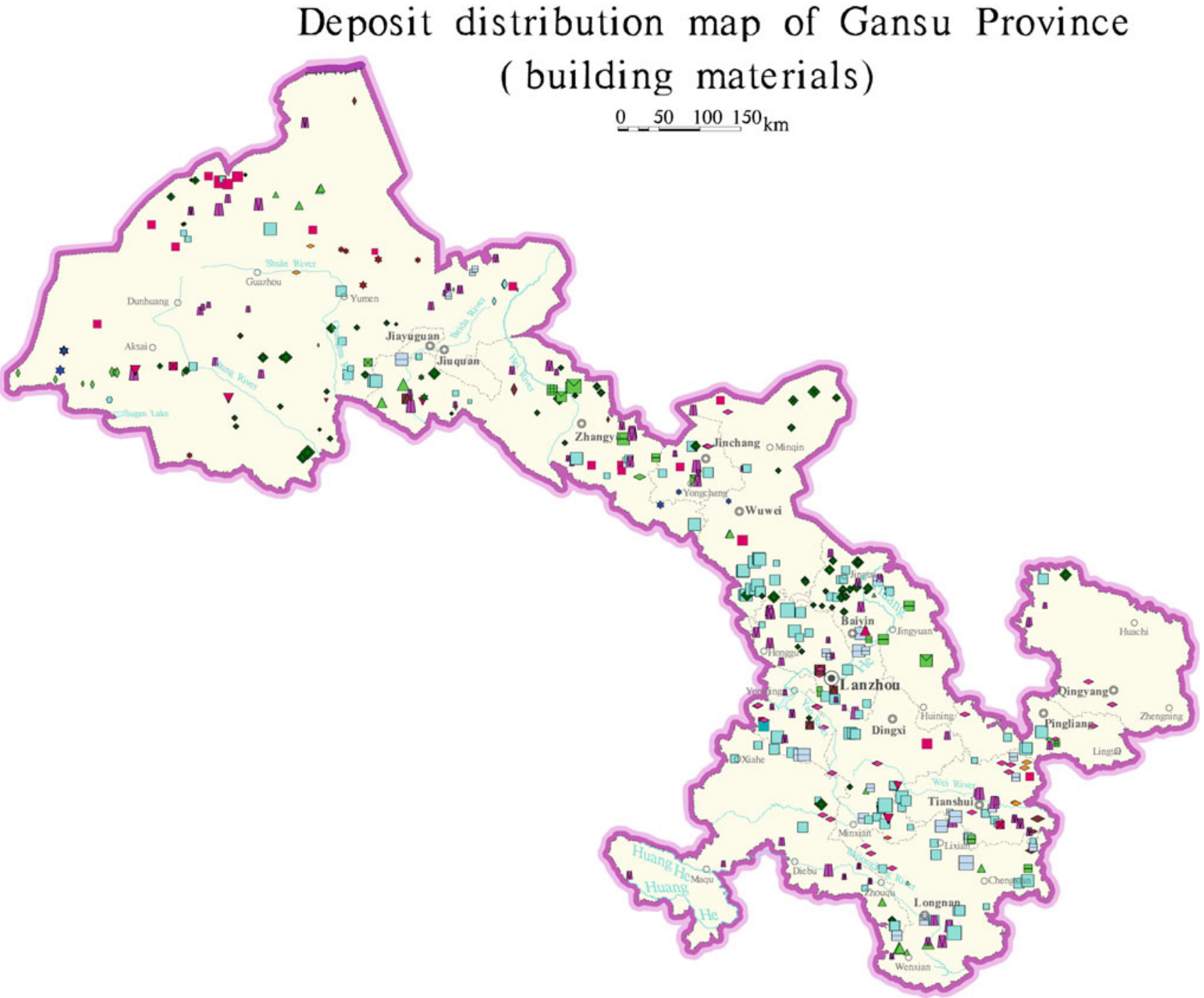




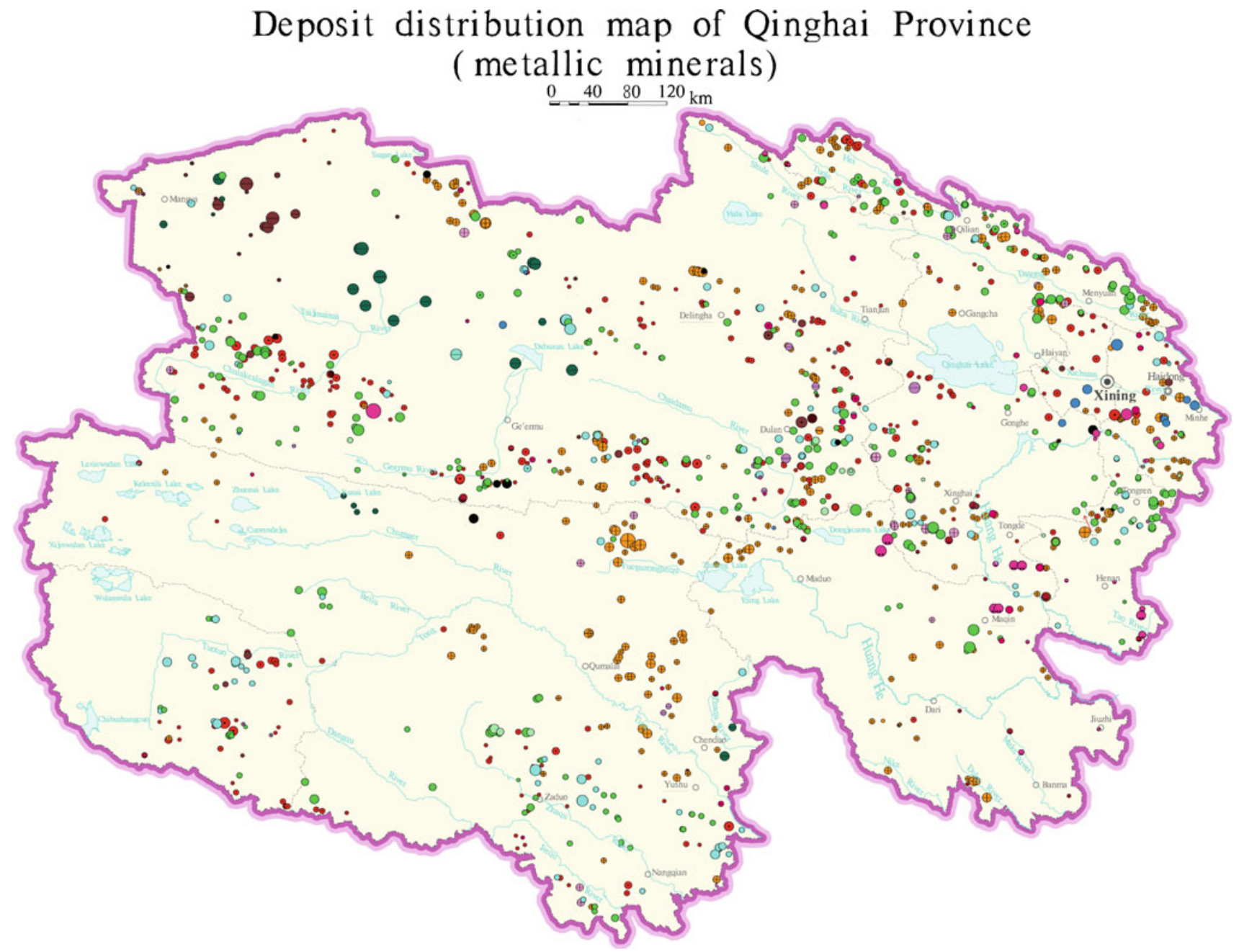




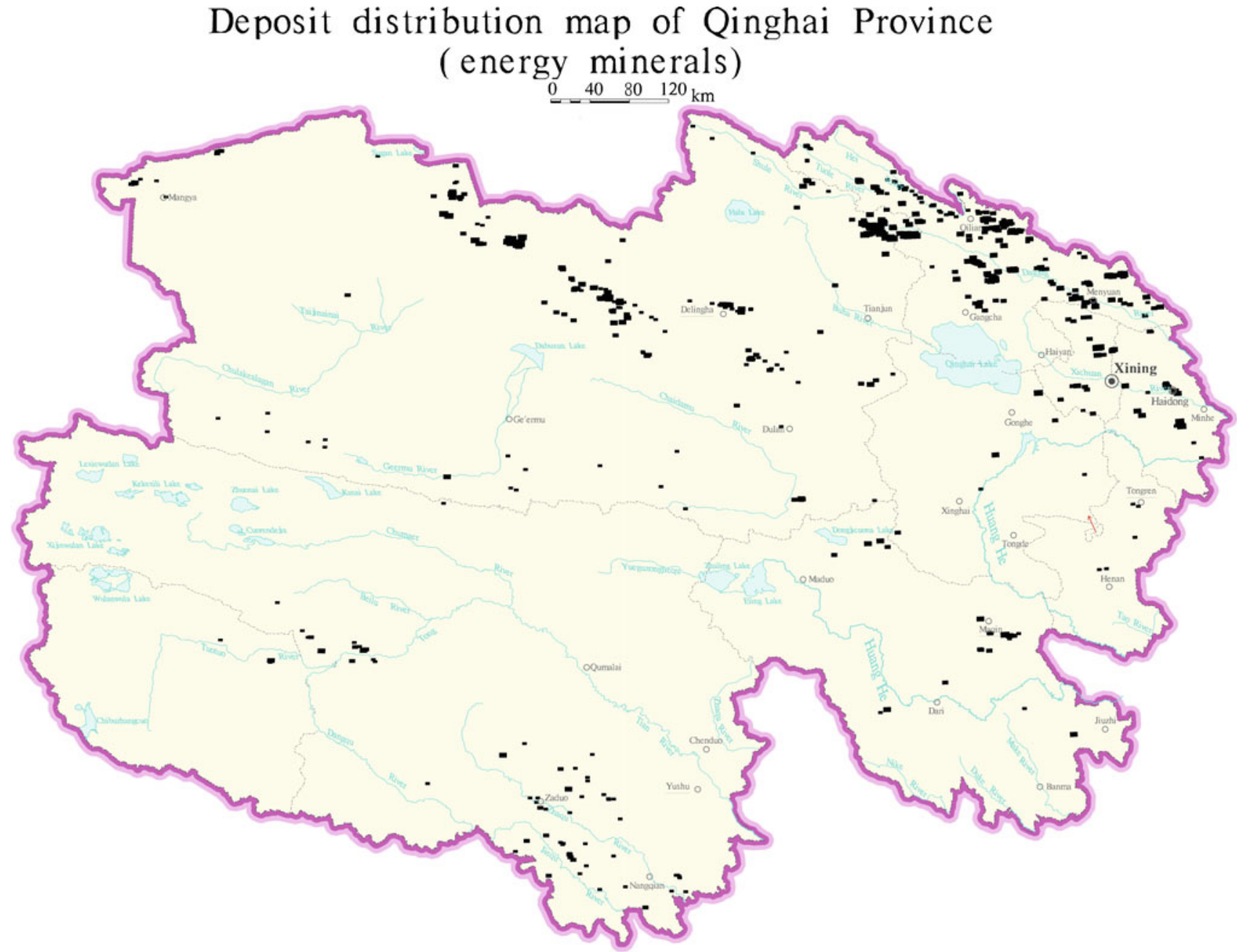




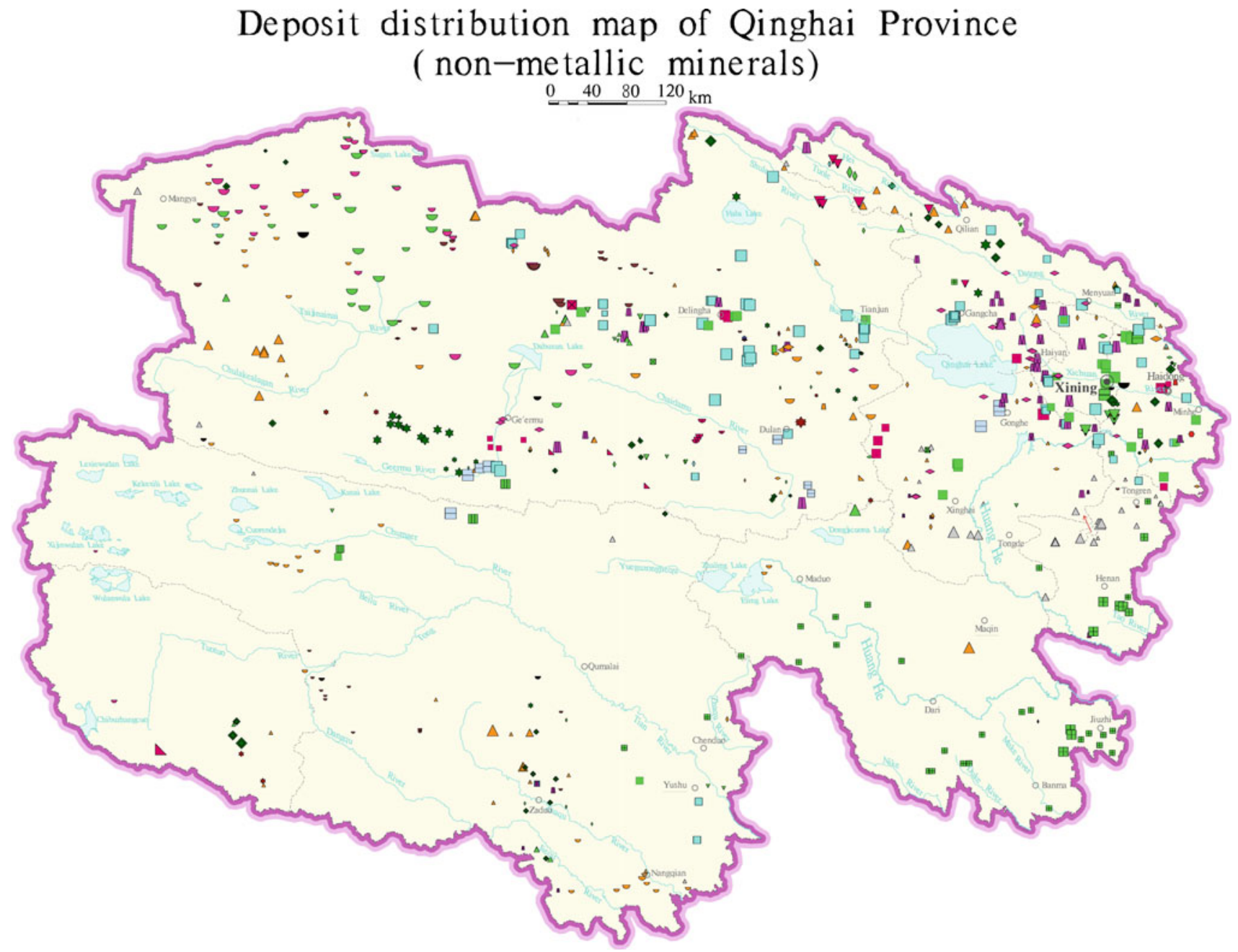


Deposit distribution map of Ningxiahuizuzizhiqu Region

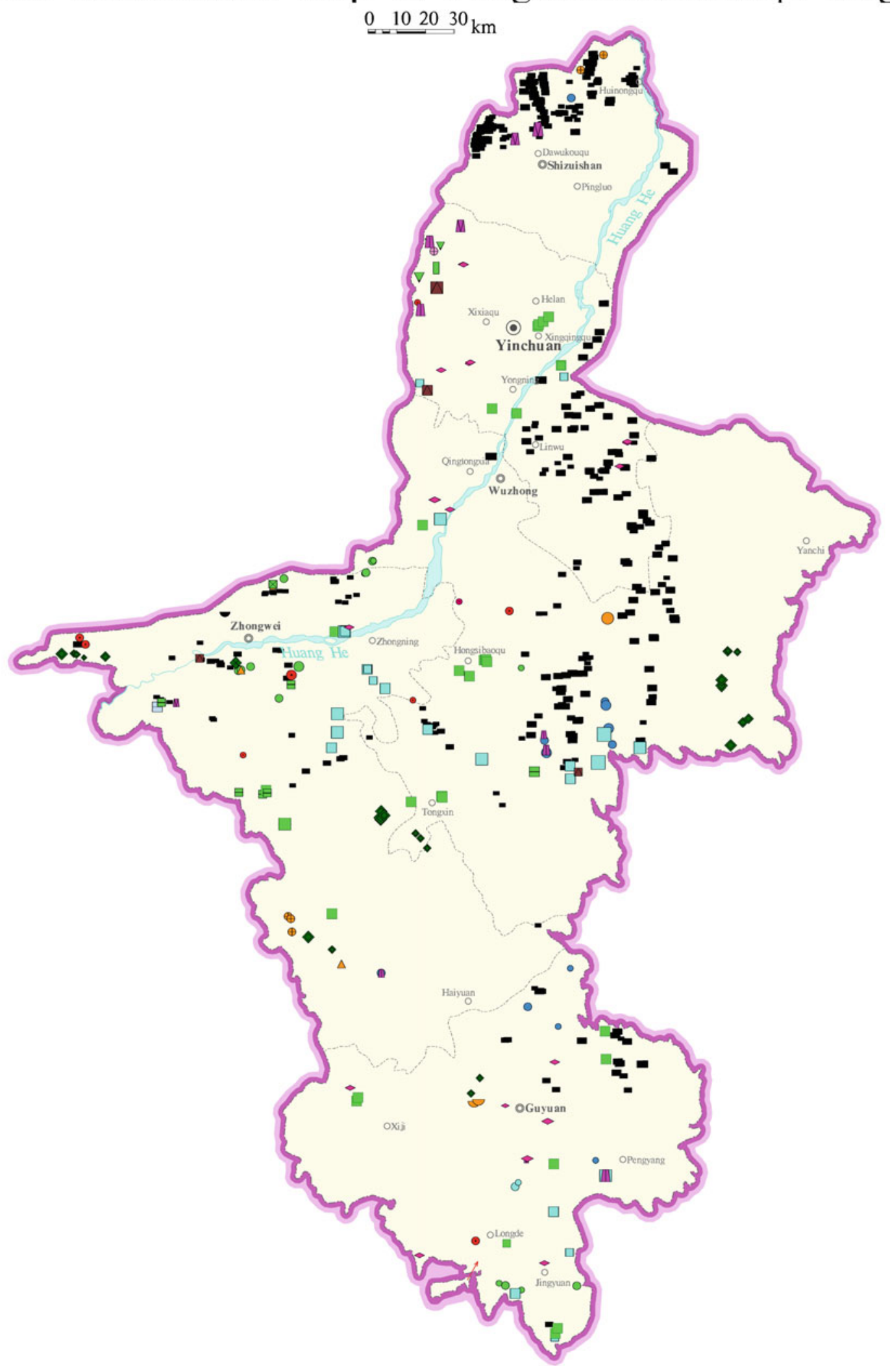


Deposit distribution map of Xinjianguygurzizhiqu Region (metallic minerals)

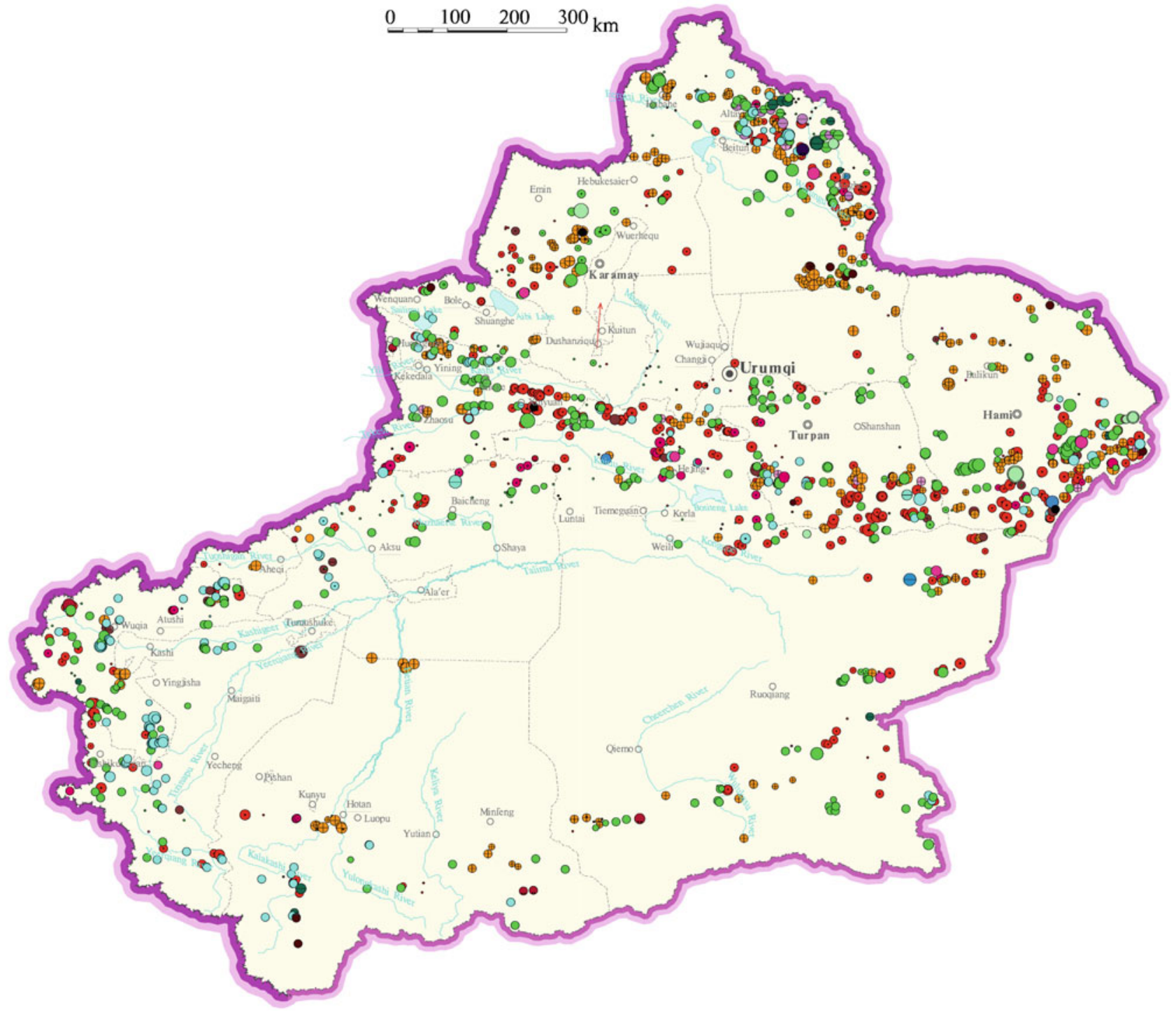


Deposit distribution map of Xinjianguygurzizhiqu Region ( energy minerals)

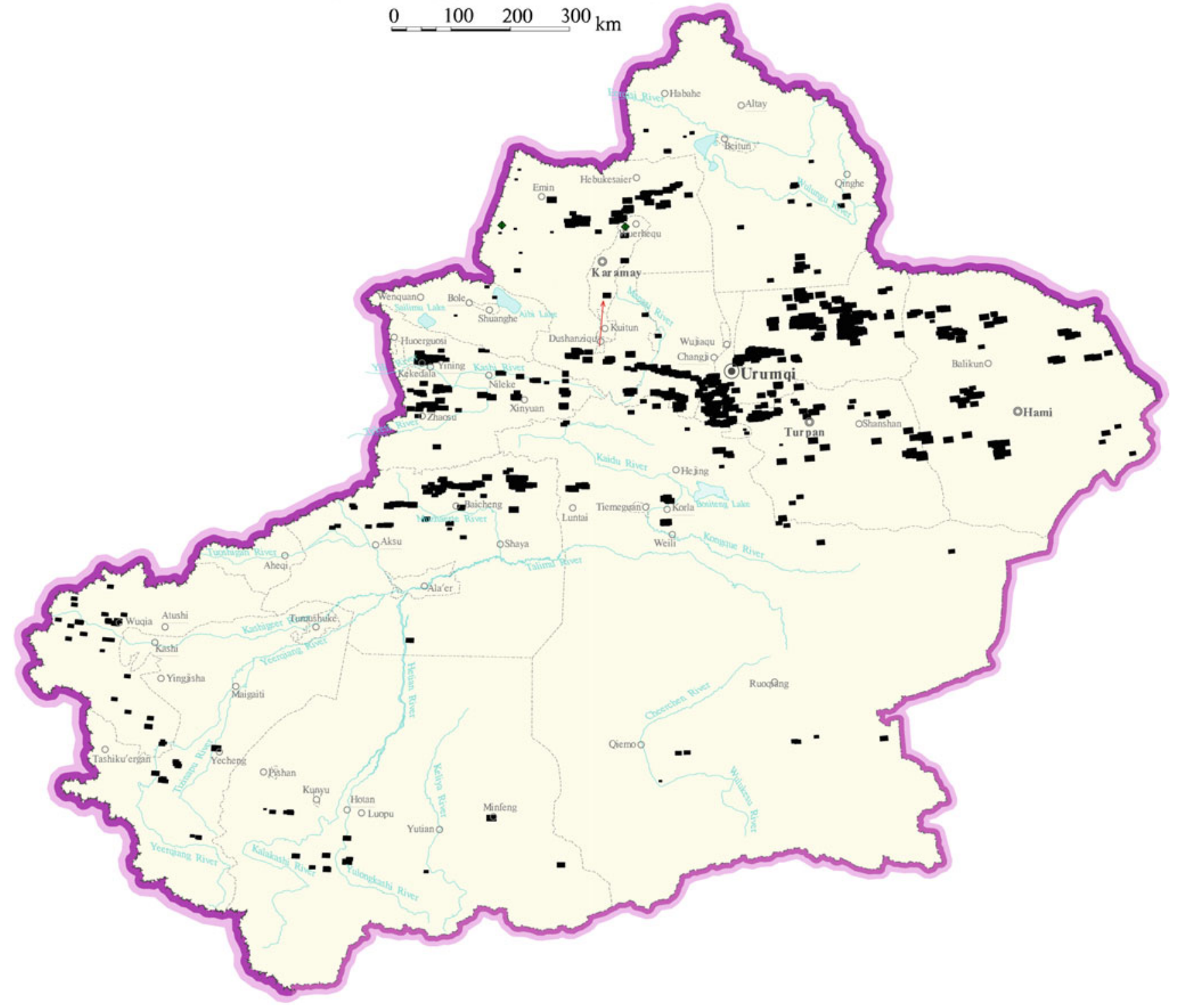


Deposit distribution map of Xinjianguygurzizhiqu Region (other non-metallic minerals)

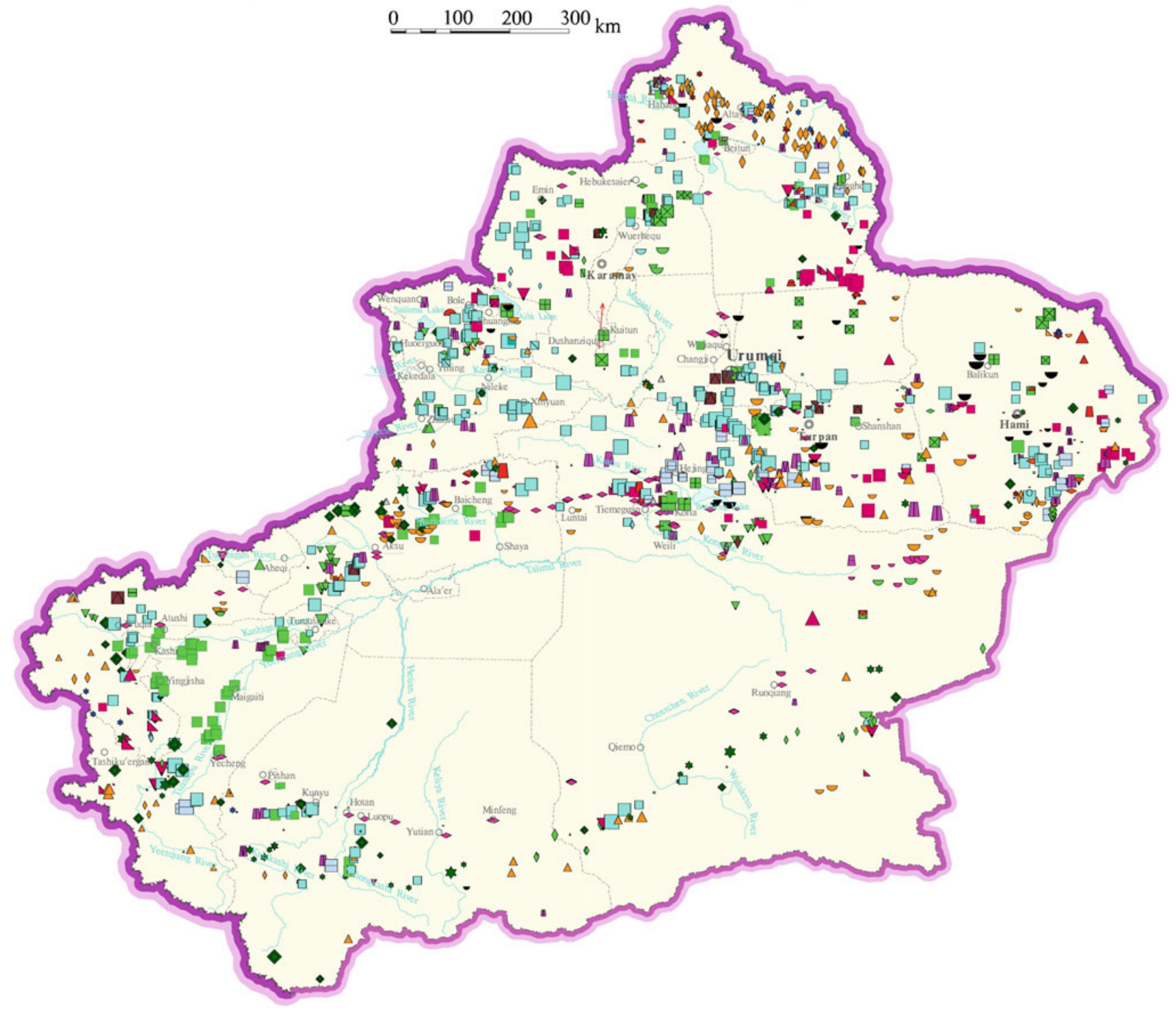




\section{Deposit distribution map of Xinjianguygurzizhiqu Region (building materials)}

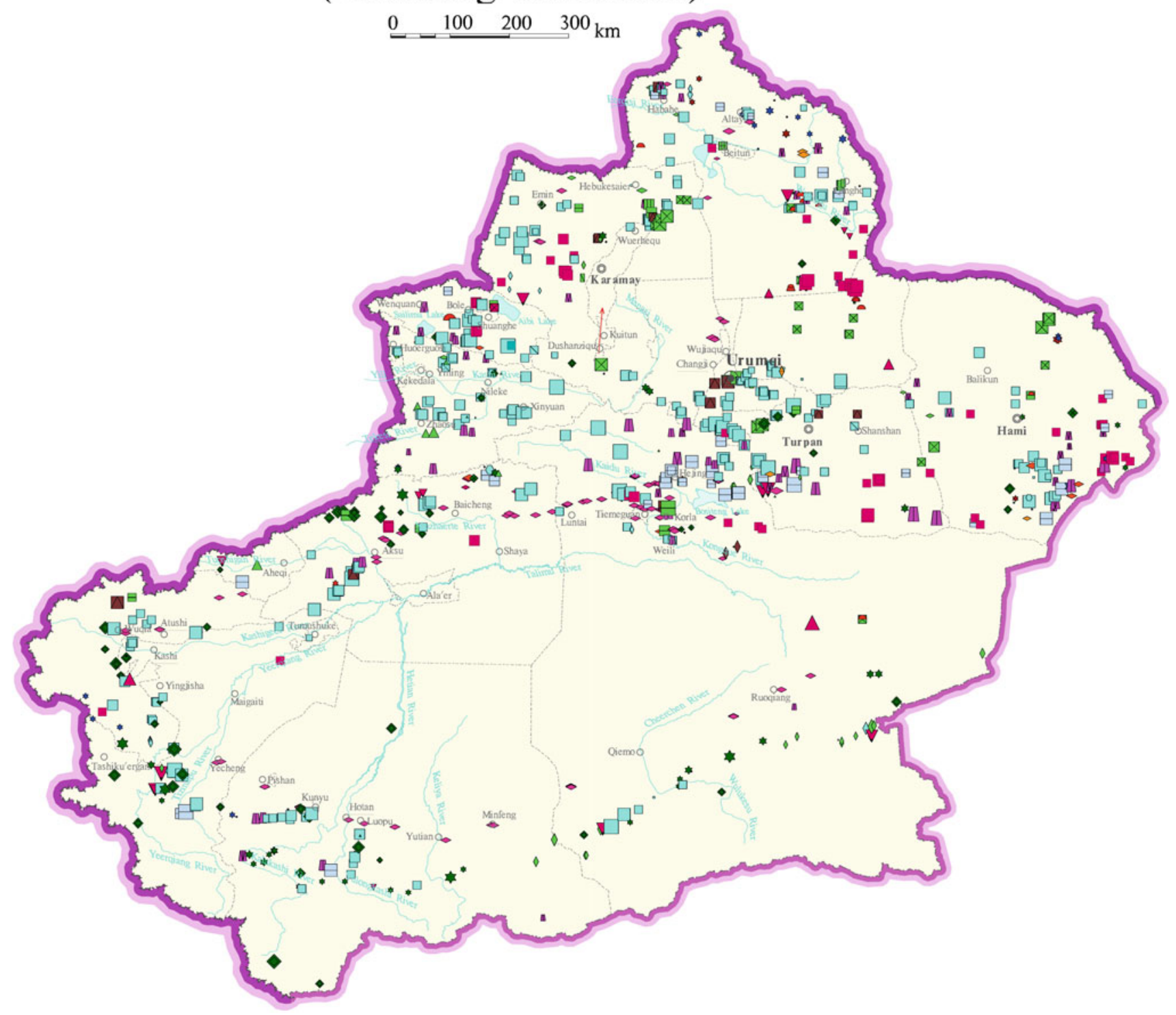

Open Access This chapter is licensed under the terms of the Creative Commons Attribution 4.0 International License (http:// creativecommons.org/licenses/by/4.0/), which permits use, sharing, adaptation, distribution and reproduction in any medium or format, as long as you give appropriate credit to the original author(s) and the source, provide a link to the Creative Commons license and indicate if changes were made.
The images or other third party material in this chapter are included in the chapter's Creative Commons license, unless indicated otherwise in a credit line to the material. If material is not included in the chapter's Creative Commons license and your intended use is not permitted by statutory regulation or exceeds the permitted use, you will need to obtain permission directly from the copyright holder.

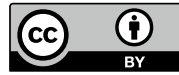

